\title{
Byzantina Symmeikta
}

Vol 3 (1979)

\section{SYMMEIKTA 3}

EONIKON IDPYMA EPEYNSN KENTPON BYZANTINQN EPEYNQN

$\Sigma \Upsilon$ M M E I K T A

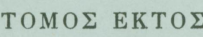

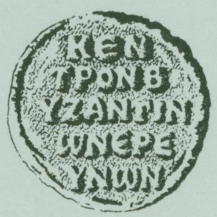

$A \Theta H N A 1985$

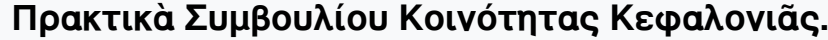

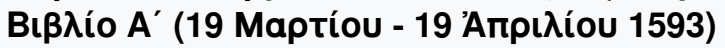

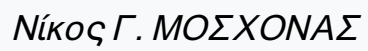

doi: $\underline{10.12681 / \text { byzsym.667 }}$

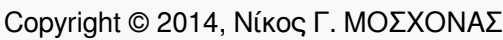

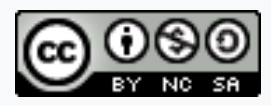

This work is licensed under a Creative Commons Attribution-NonCommercialShareAlike 4.0.

To cite this article:

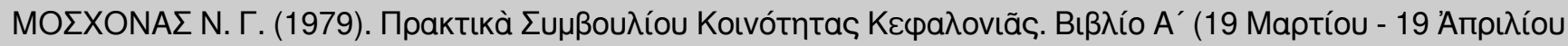
1593). Byzantina Symmeikta, 3, 265-350. https://doi.org/10.12681/byzsym.667 


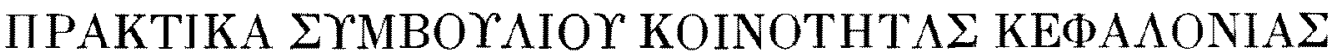

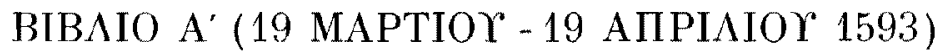

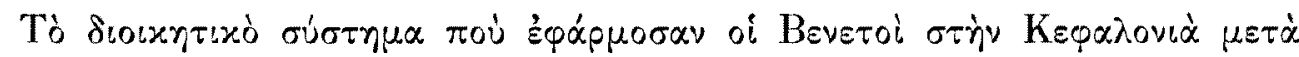

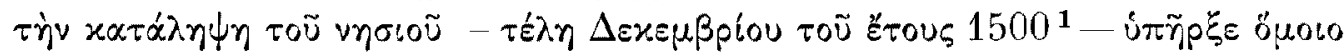

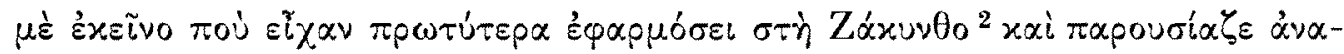

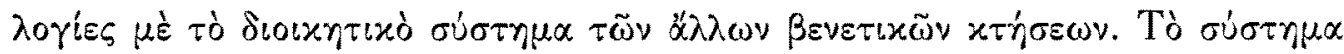

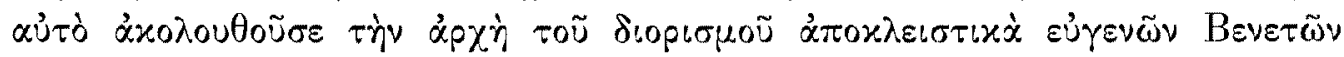

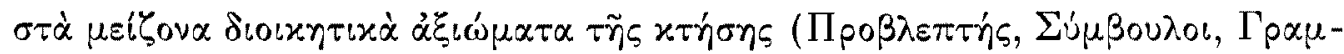

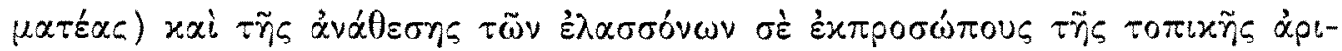

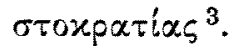

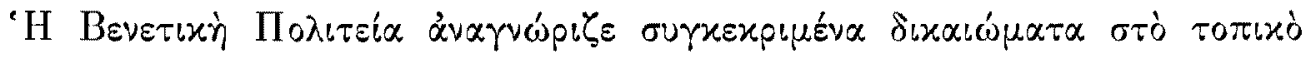

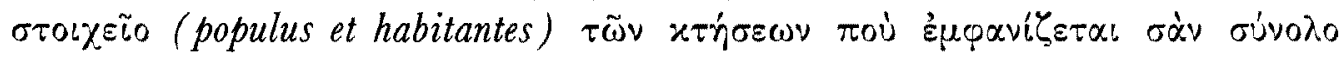

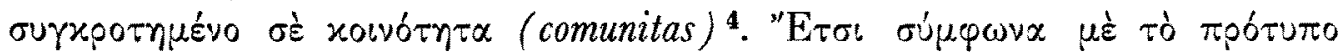

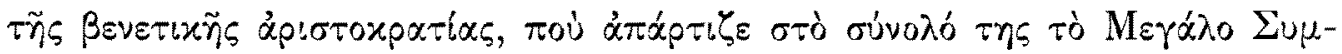

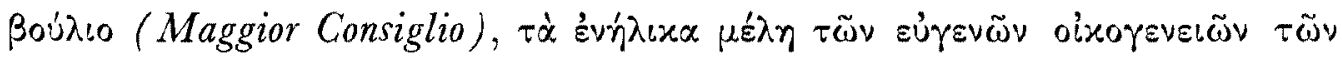

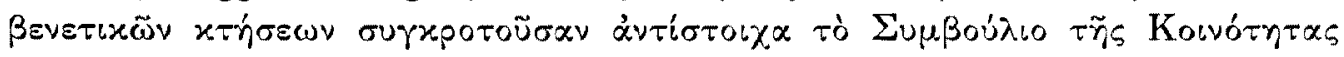

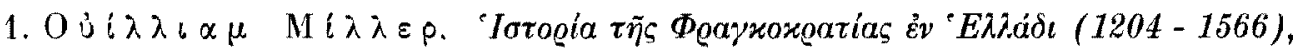

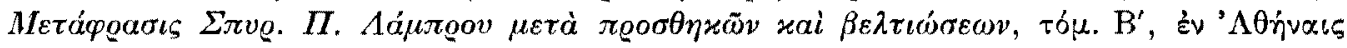

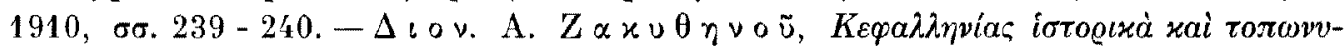

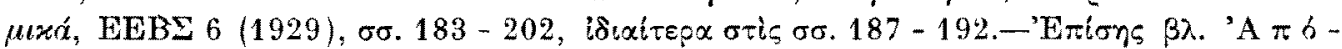

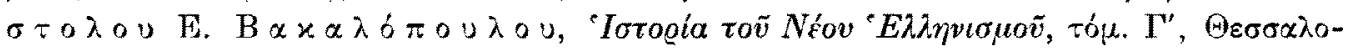

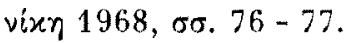

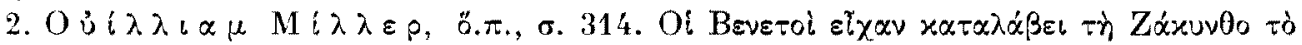

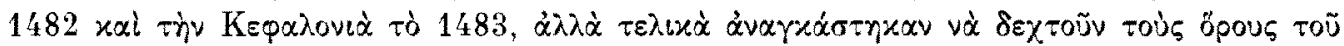

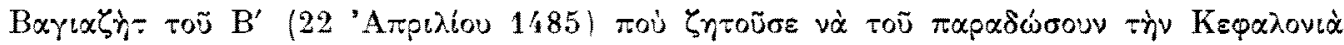

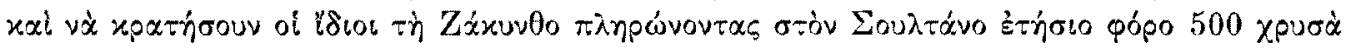

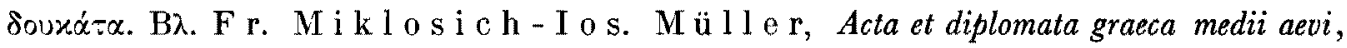

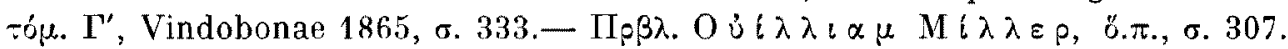

3. Ermanno Lunzi, Della condizione politica delle Isole fonie sotto il dominio veneto. . Versione con note di Marino Dr. Typaldo-Foresti e Nicolò Barozzi riveduta ed aumentata dall' autore, Venezia $1858, \sigma .313$ к. $\pi$.

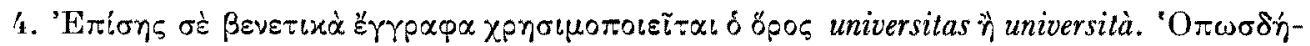

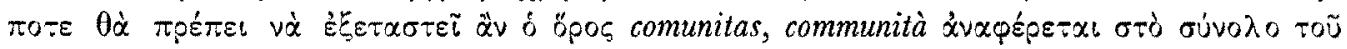

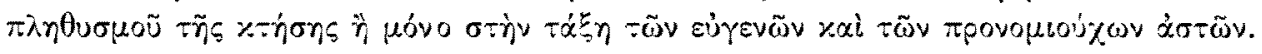




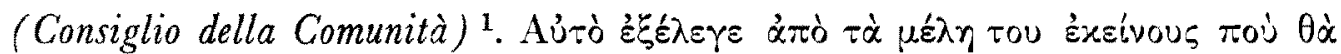

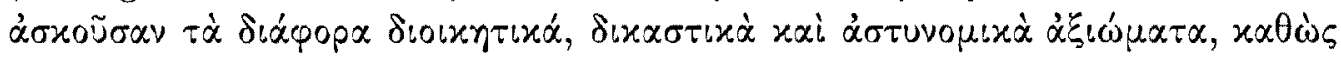

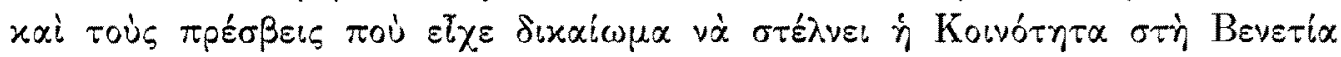
$\gamma\llcorner\dot{\alpha} \tau \dot{\eta} \delta \varepsilon \varepsilon u \theta \dot{\varepsilon} \tau \eta \eta \eta$

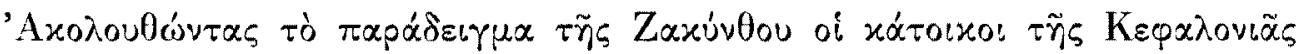

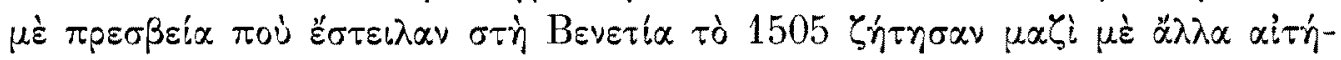

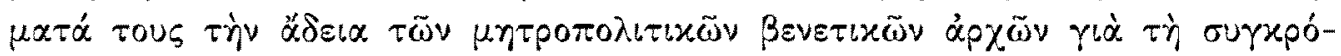

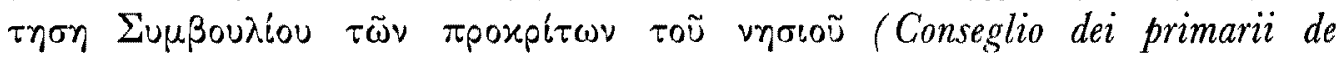

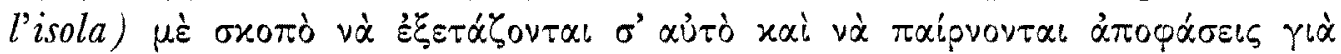

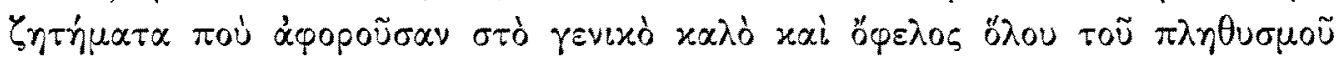

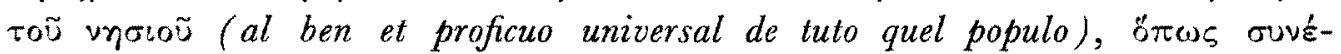

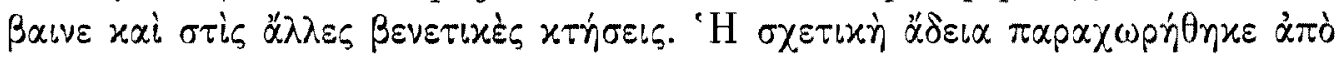

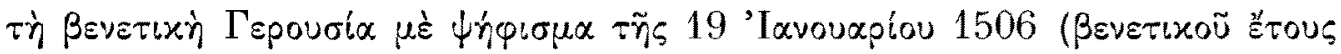

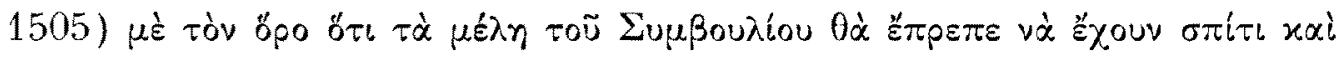

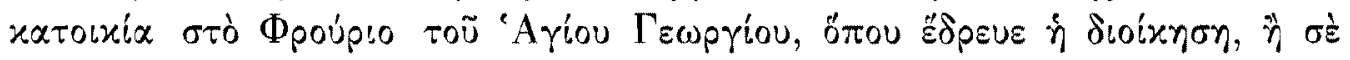

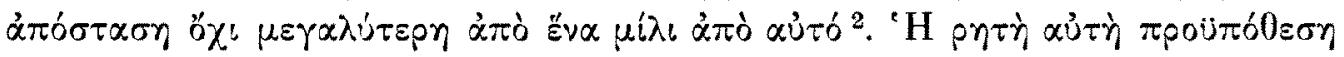

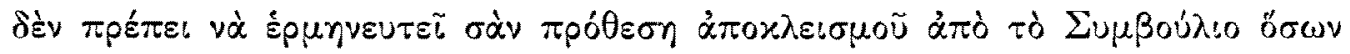

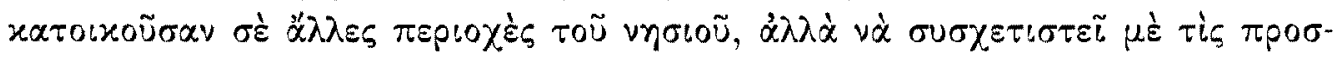

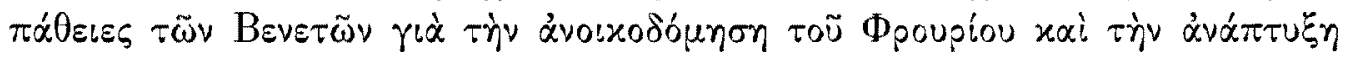

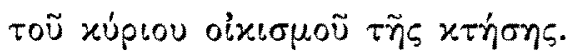

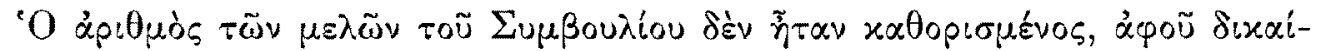

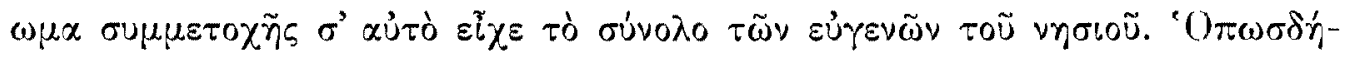

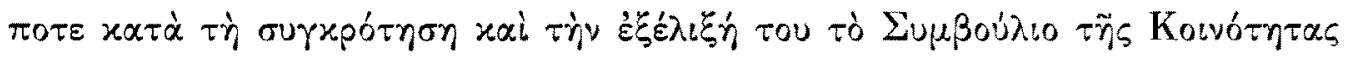

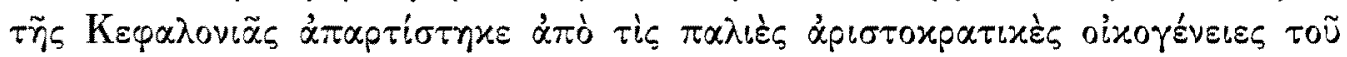

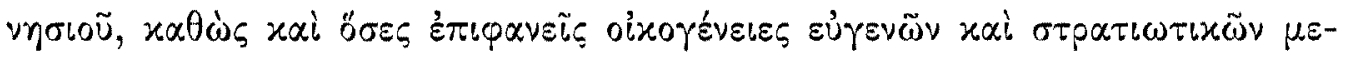

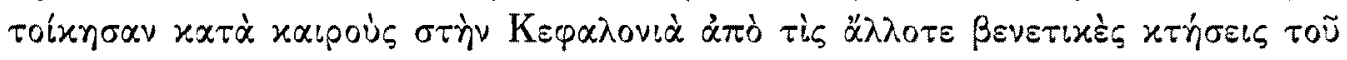

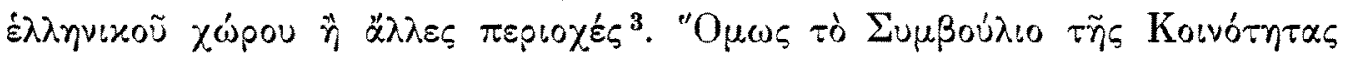

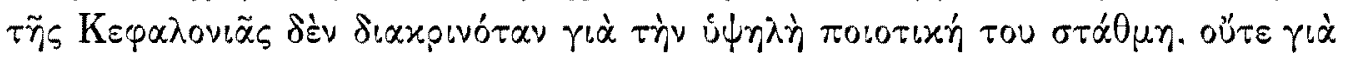

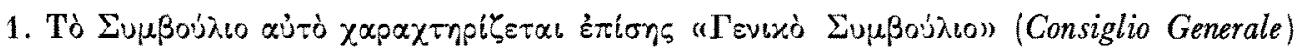

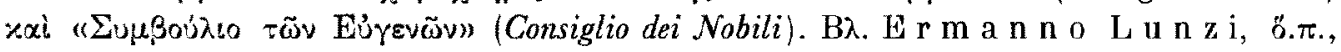

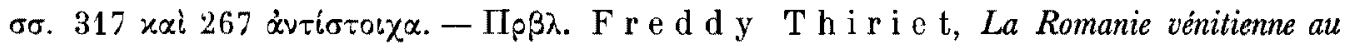

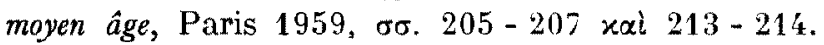

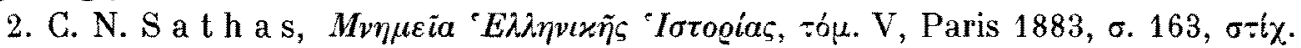

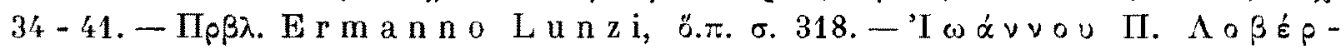

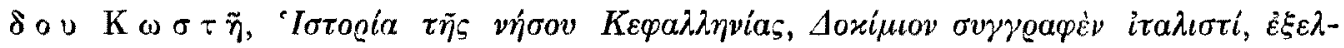

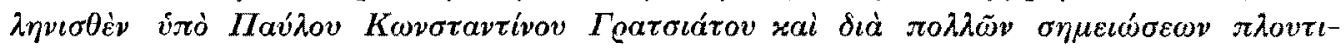

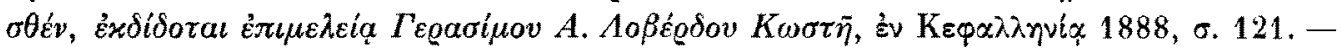

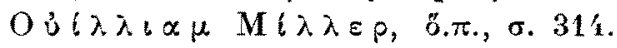

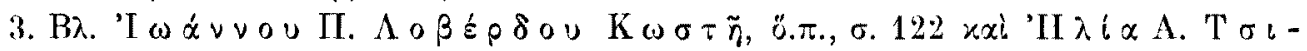

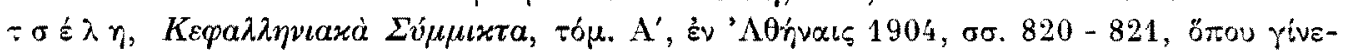

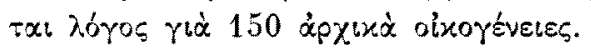




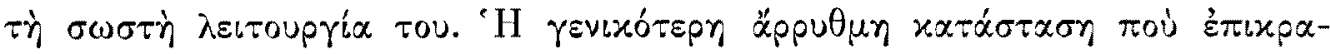

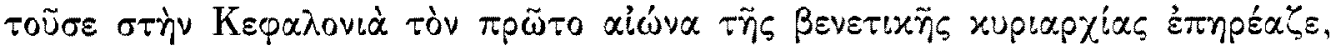

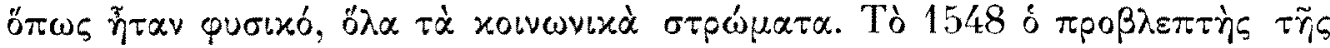

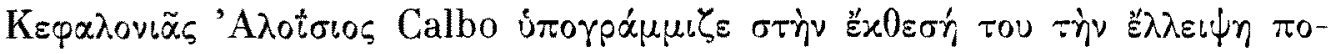

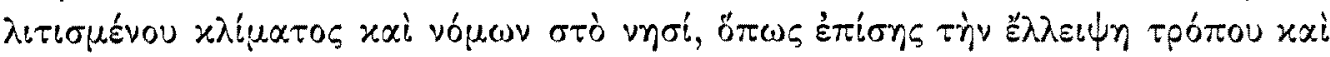

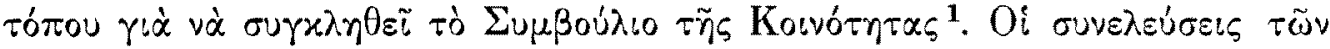

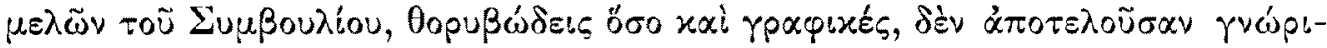

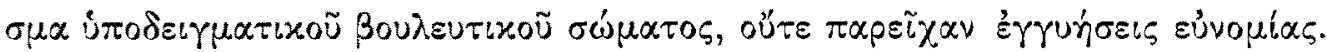

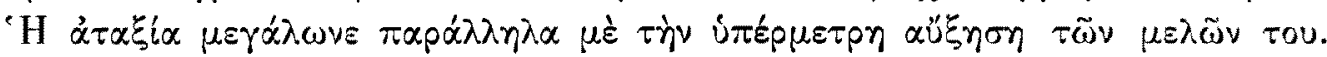

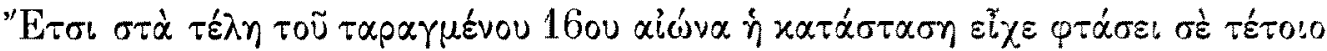

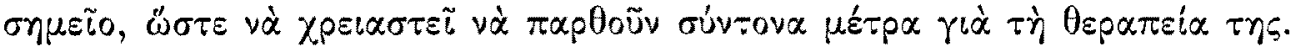

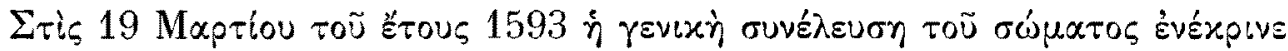

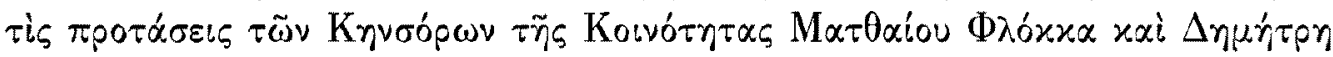

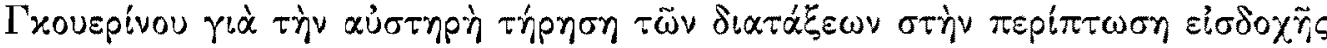

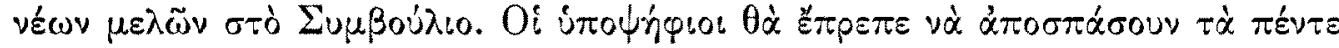

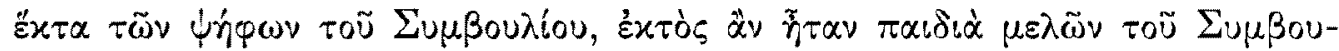

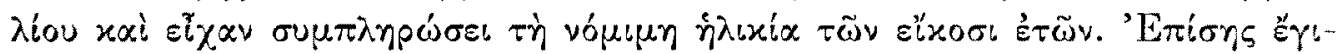

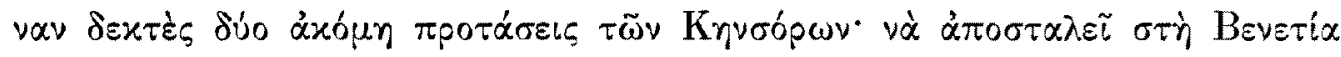

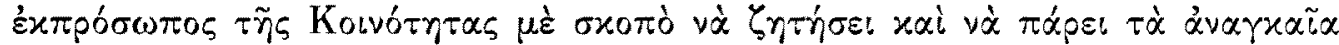

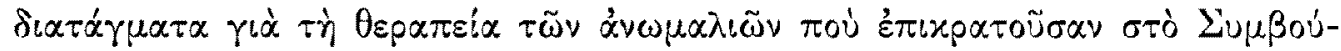

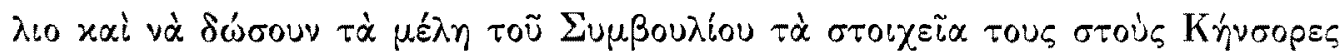

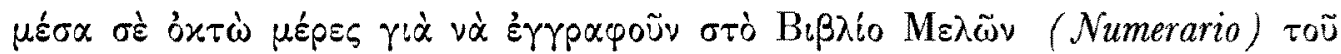

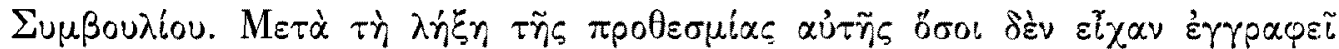

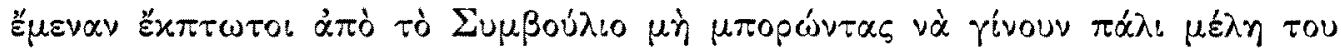

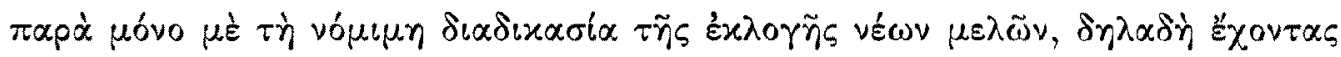

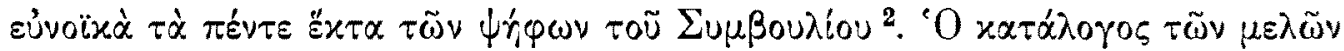

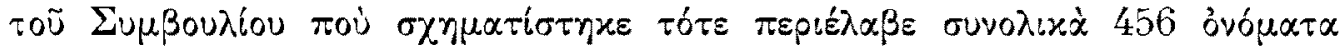

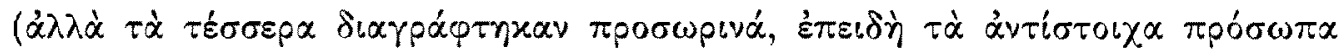

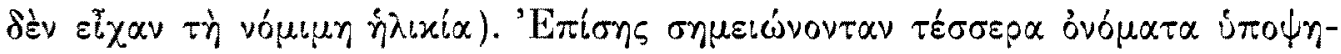
pi $\omega \nu \mu \varepsilon \lambda \tilde{\omega} \nu^{3}$.

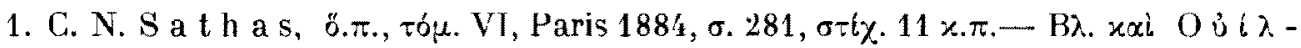
$\lambda \iota \propto \mu \mathrm{M} i \lambda \lambda \varepsilon \rho$, ö. $\pi . \sigma .314$.

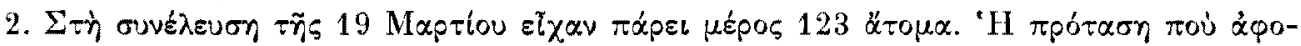

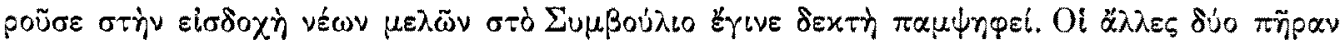

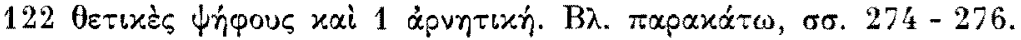

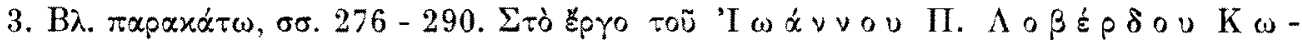

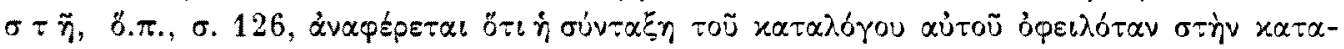

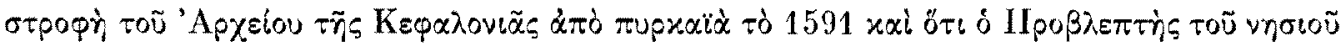

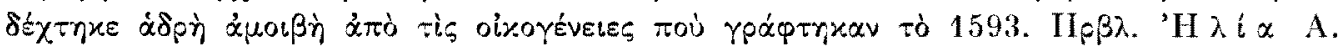

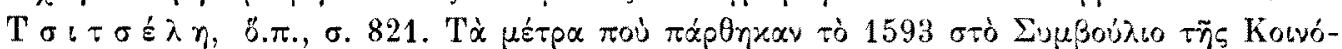

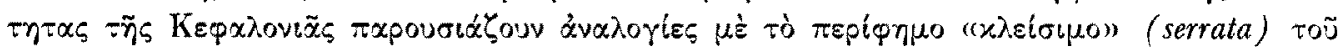




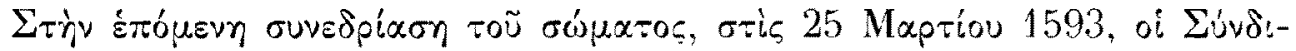

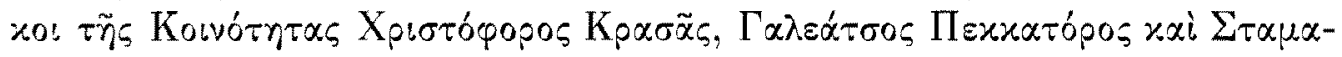

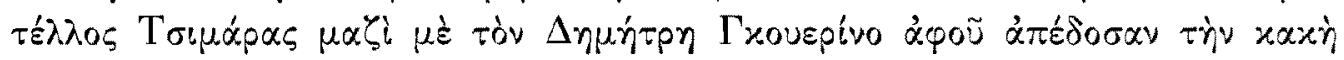

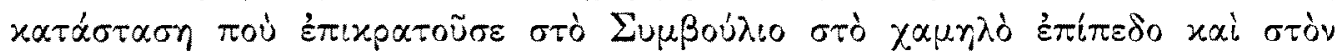

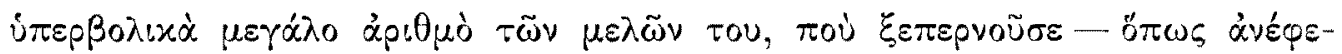

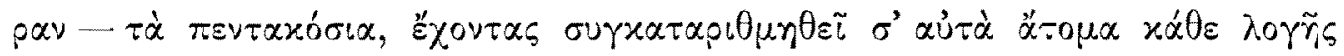

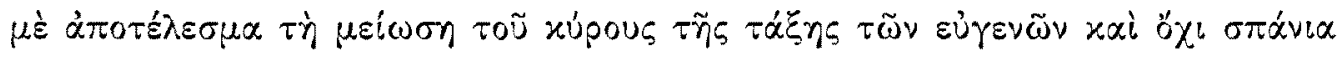

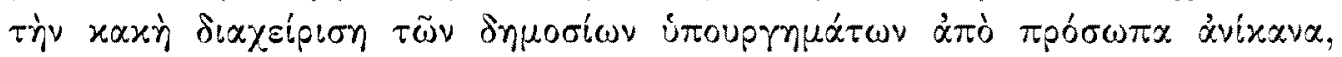

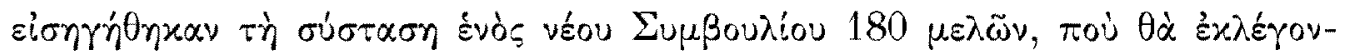

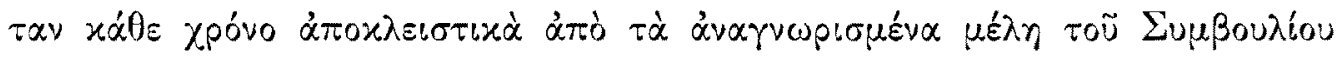

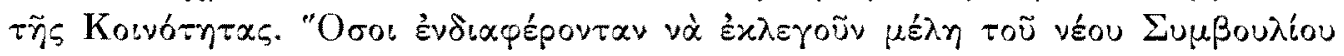

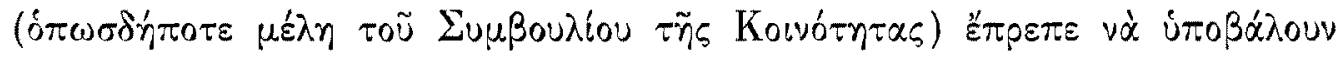

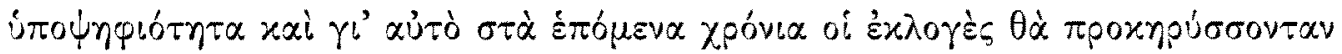

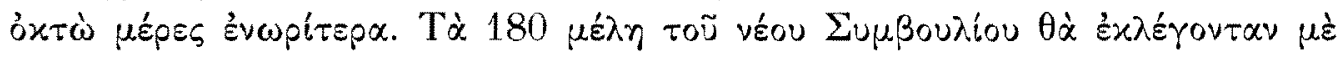

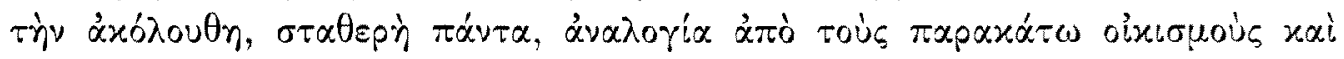

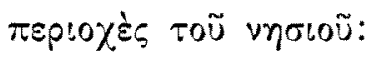

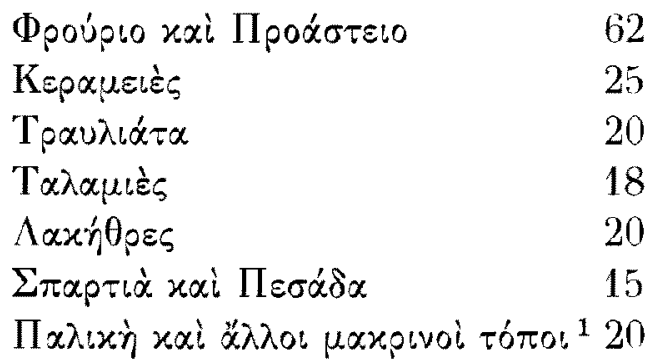

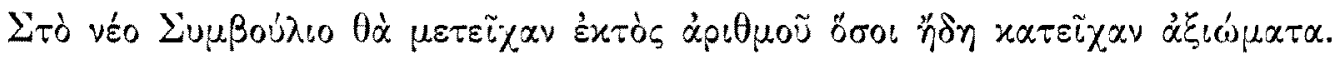

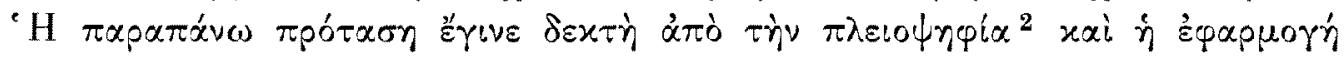

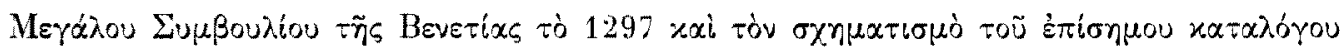

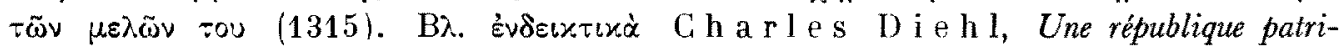

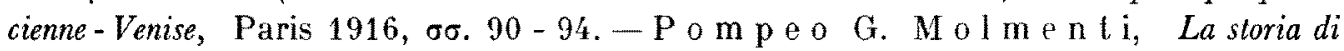

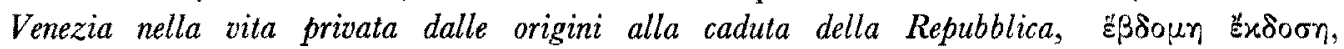

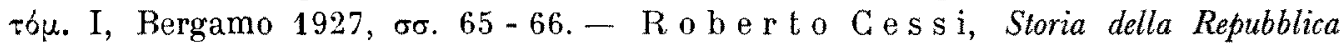

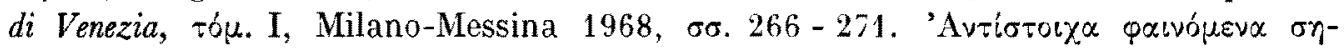

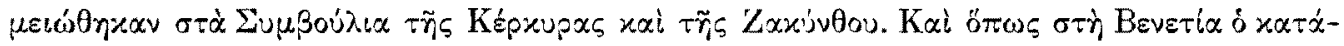

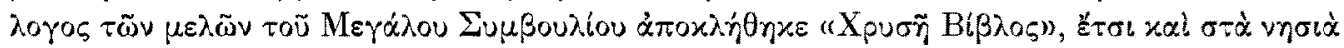

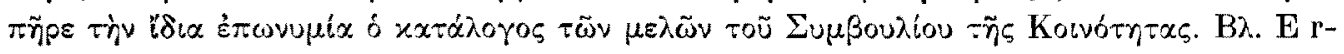

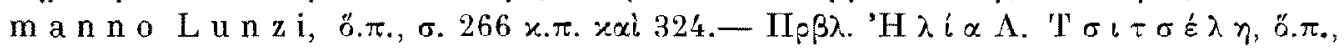
б. $820 x . \pi$.

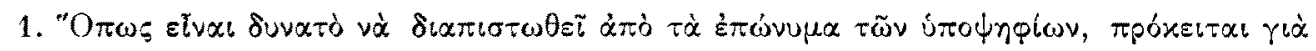

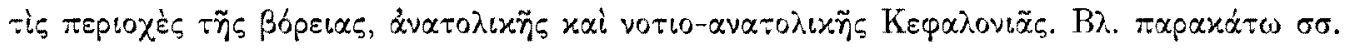
$298-299$.

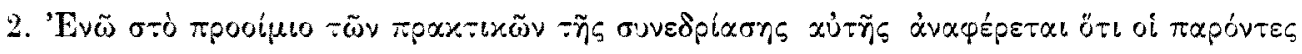




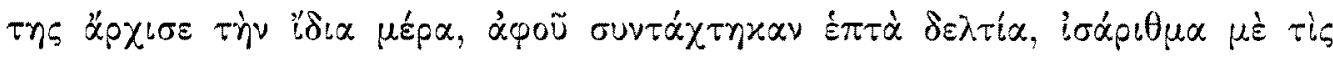

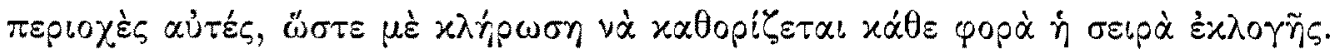

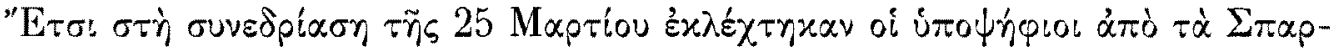

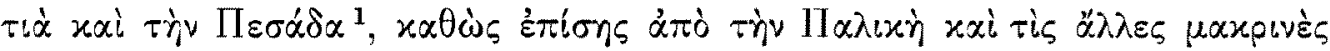

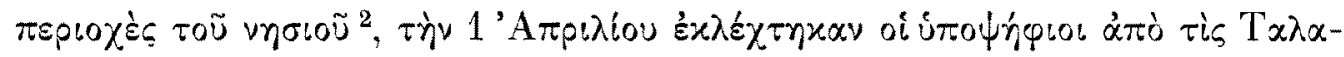

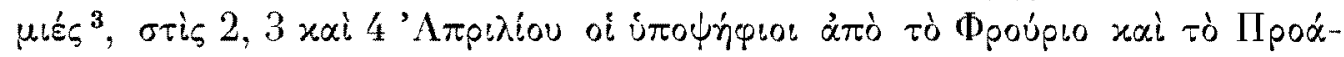

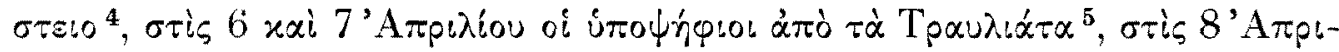

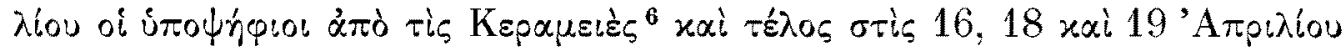

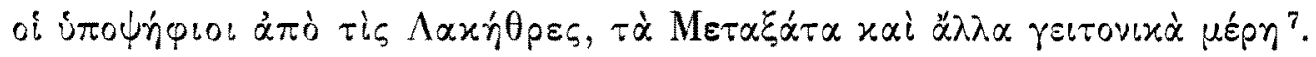

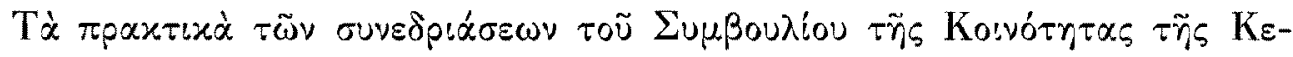

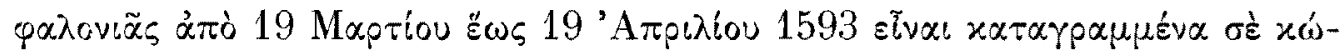

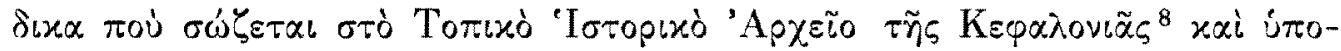

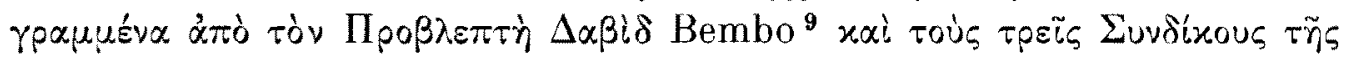

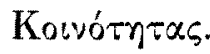

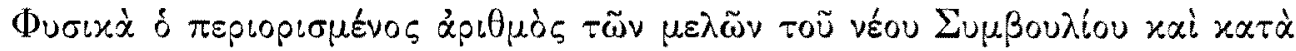

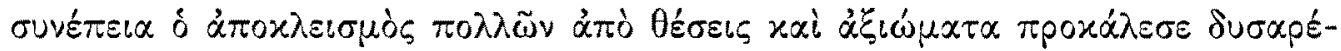

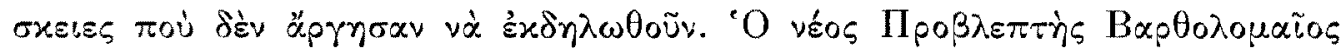

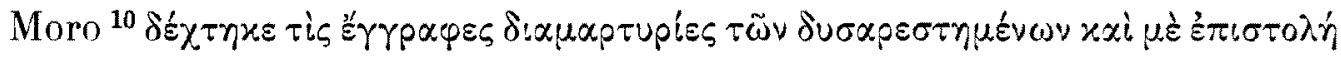

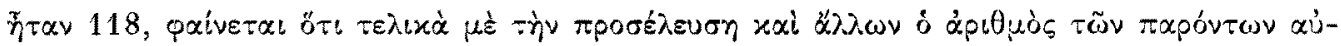

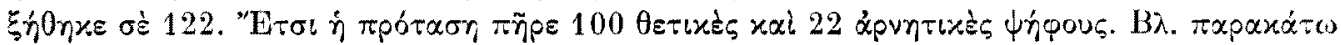

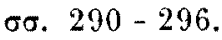

1. $B \lambda . \pi \alpha \rho \alpha x \dot{\alpha} \tau \omega \sigma \sigma .296-297$.

2. $\mathrm{B \lambda}$. $\pi \alpha \rho \alpha \alpha \alpha \dot{\alpha} \sigma \sigma \sigma .298-299$.

3. $\mathrm{B \lambda} . \pi \propto р \alpha x \alpha \dot{\alpha}=\omega \sigma \sigma .300-301$.

4. $B \lambda . \pi \propto р \alpha \alpha \alpha<\omega \sigma \sigma .301-306$.

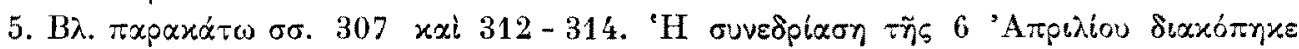

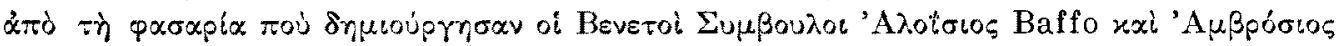

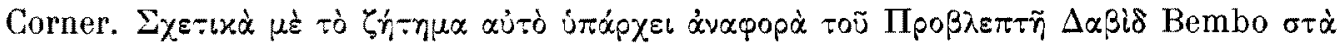

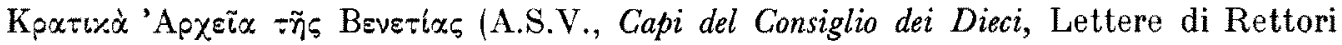

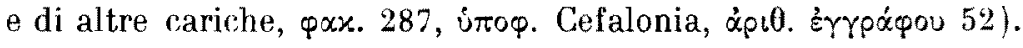

6. $B \lambda . \pi x p \alpha x \alpha \dot{\tau} \tau \sigma \sigma .314-315$.

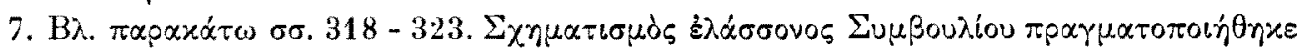

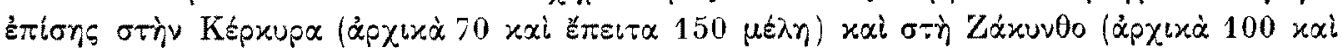

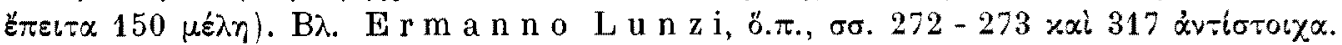

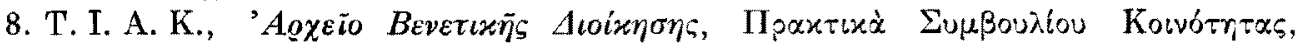
Bıß入io 1 (Consiglio della Communità, 1 ).

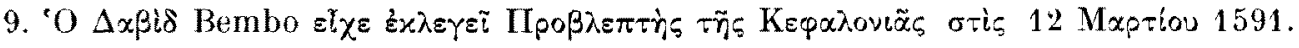

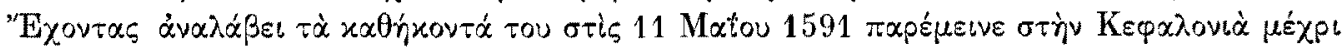
tic 10 Matou 1593 (A.S.V.. Segretario alle voci, Maggior Consiglio-Elezioni, reg. 7,

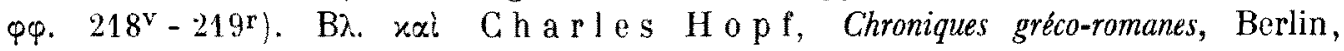
1873, , 400 .

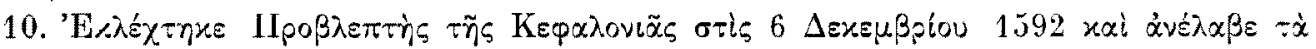

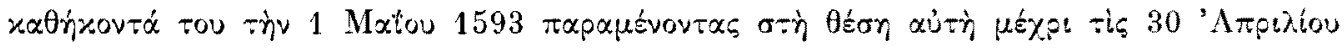




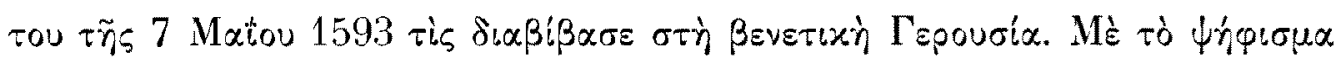

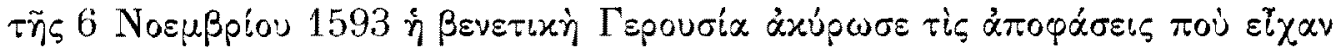

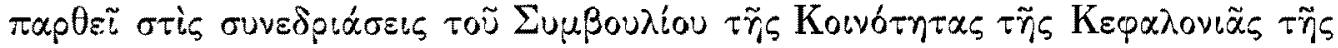

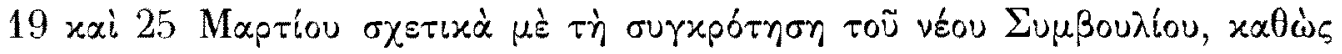

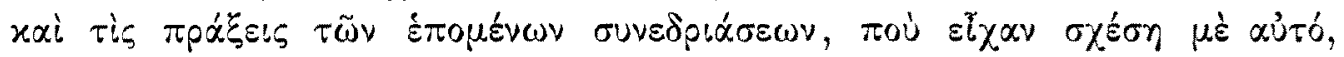

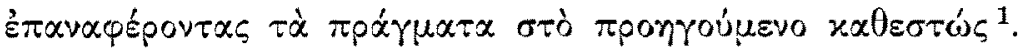

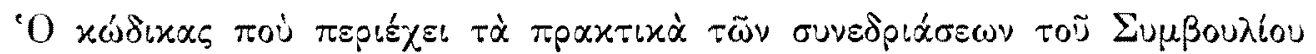

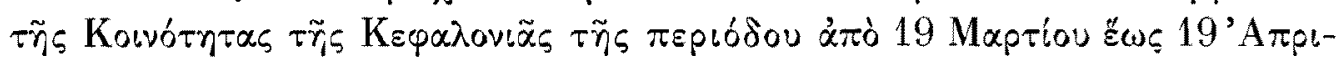

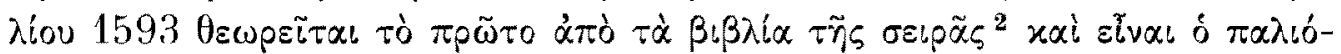

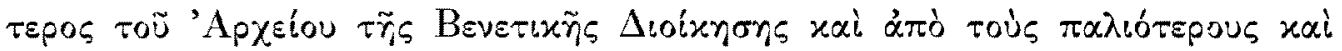

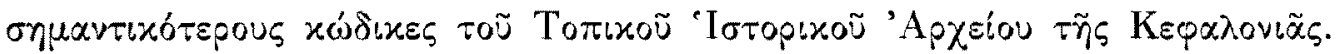

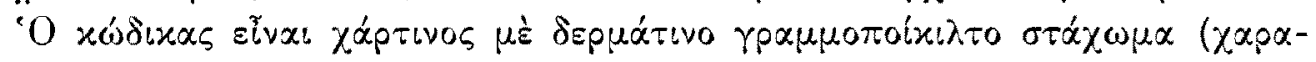

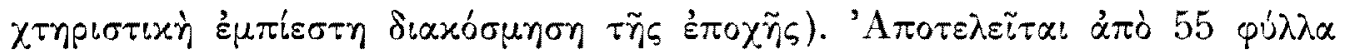

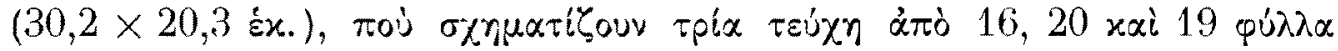

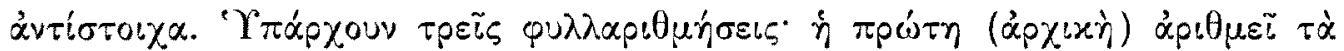

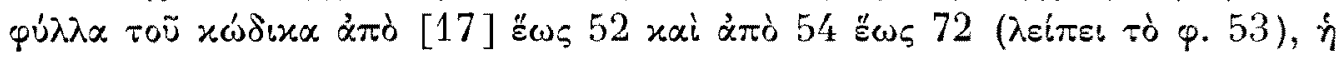

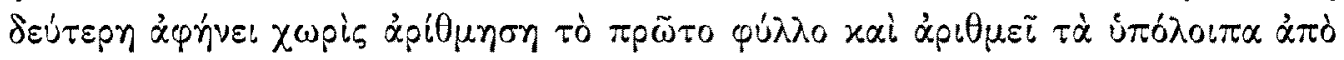

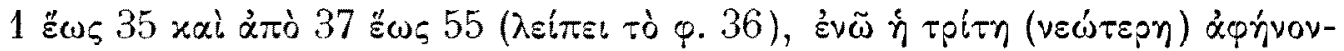

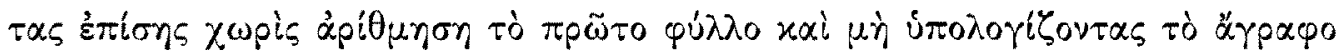

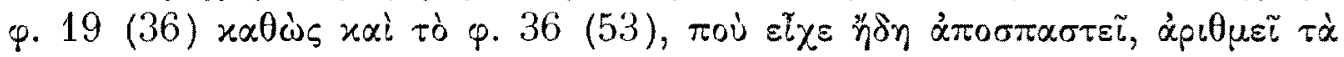

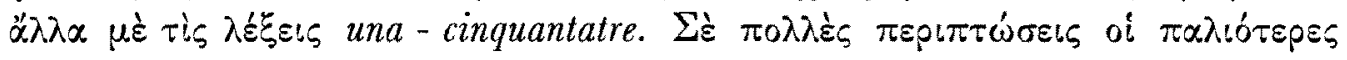

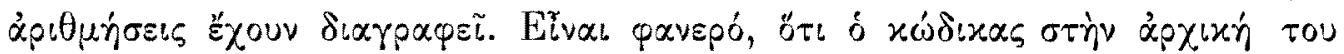

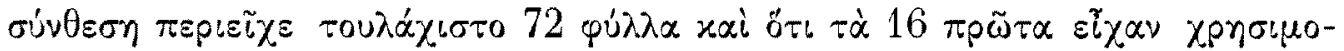

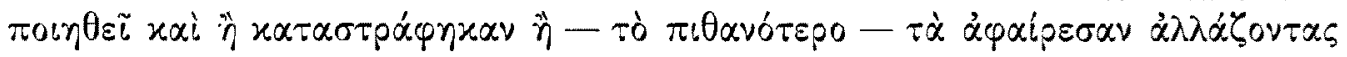

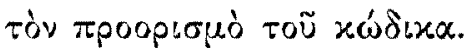

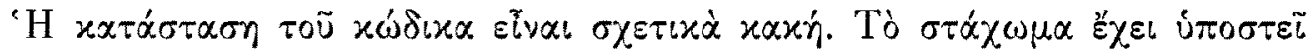

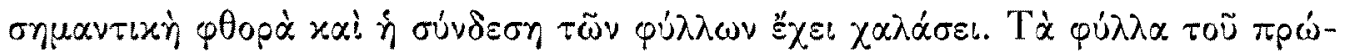

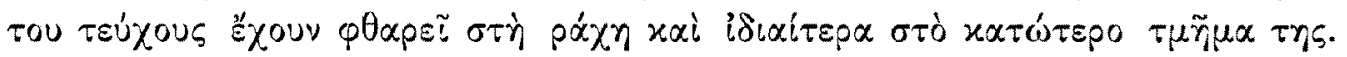

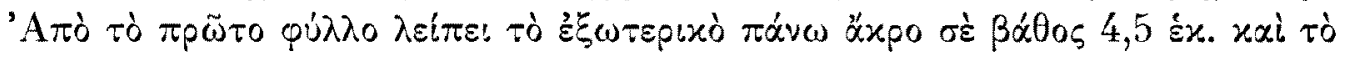

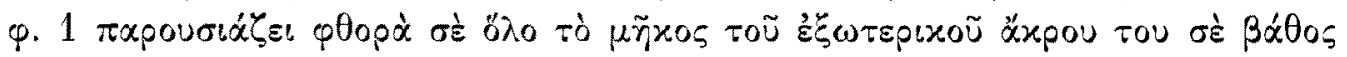

1595 (A.S.V., Segretario alle voci, Maggior Consighio-Elezioni, reg. 7, $\varphi \varphi .218^{\mathrm{v}}-$

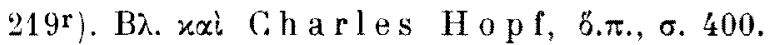

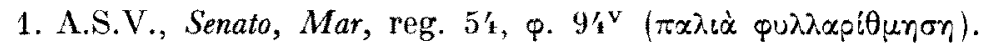

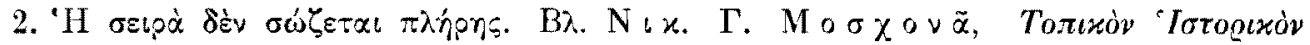

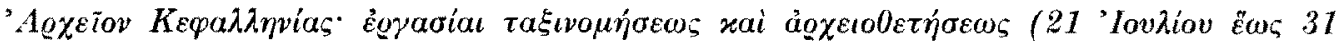

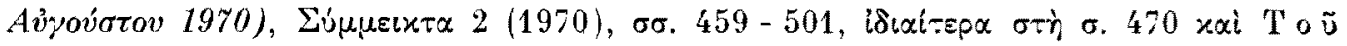

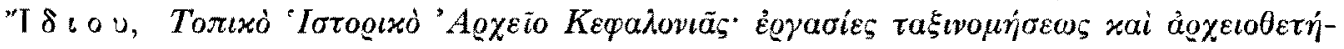

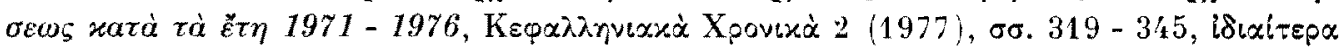
бтทे $\sigma .325$. 


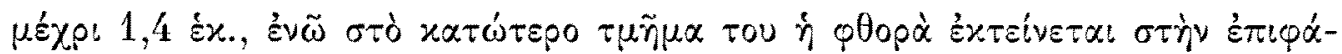

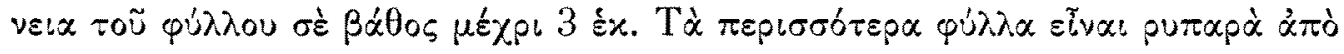

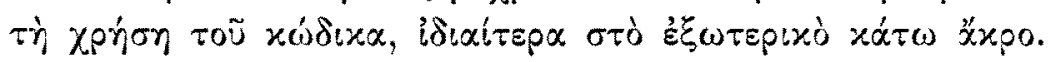

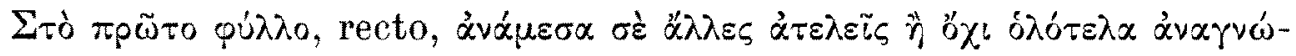

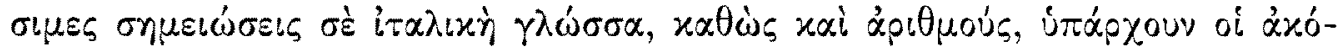

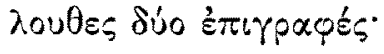

$\alpha^{\prime}$

Libro primo anticho di Cons[egli]o della Spetabile Magnifica Communità, nel tempo che era il Clarissimo signor David Bembo 15[93].

$\beta^{\prime}$

Libro di Consigli d $[$ ella] Spetabil Communità nel tempo che era il Clarissimo signor David Bembo Proveditor.

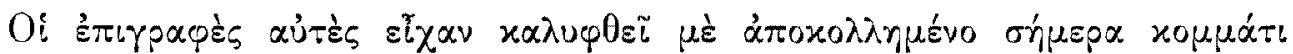

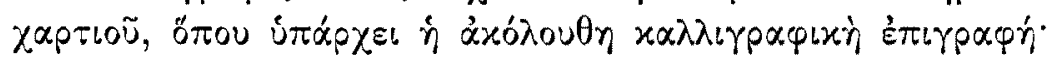

Libro $1^{\circ}$ Consigli et altro del ex-Governo Veneto. Principia li 19 marzo 1593 e termina li primo luglio 1604. Nicolas D. Troyanos.

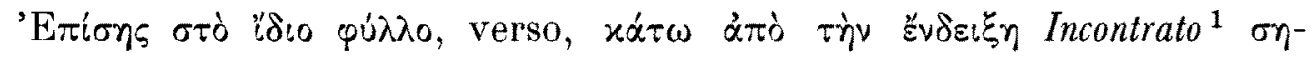
$\mu \varepsilon เ \omega^{\prime} \vee \tau \tau$ :

Libro 1. primo Consigli et altra dell'ex Governo Veneto. Principia li 19, marzo 1593 e termina li primo luglio 1604. Sono carte scritte cinquanta tre.

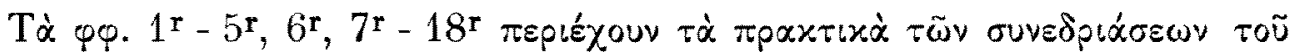

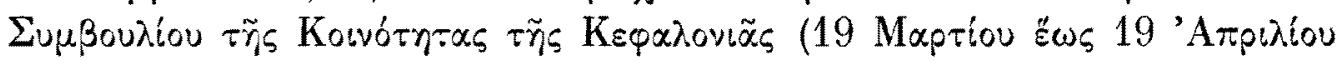

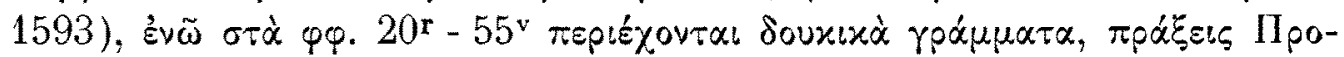

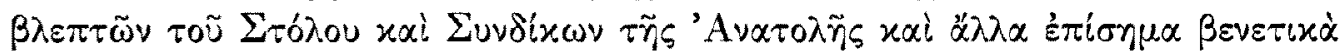

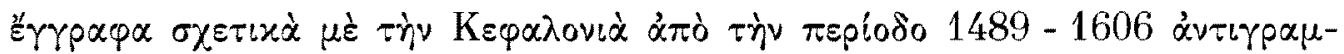

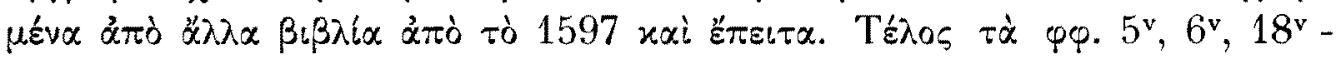

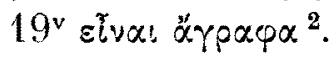

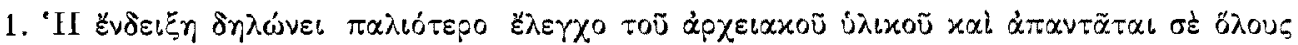

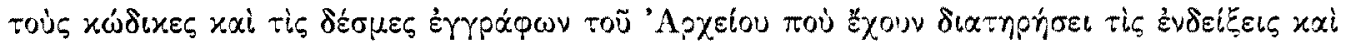

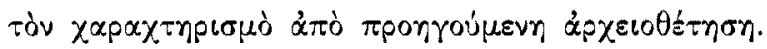

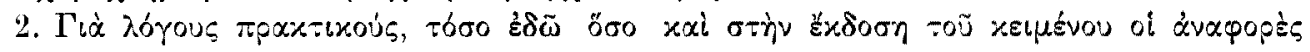

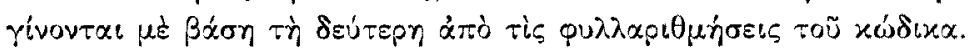




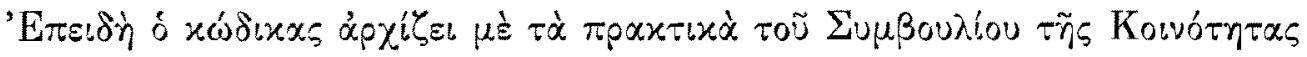

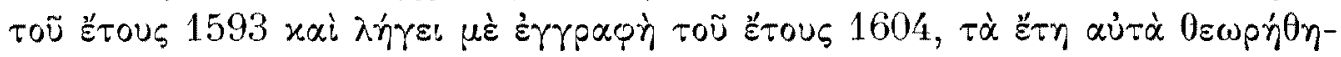

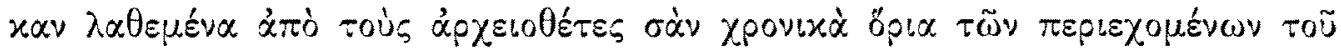

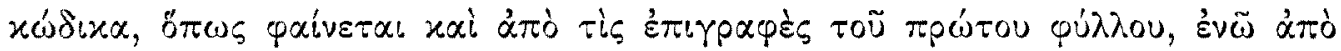

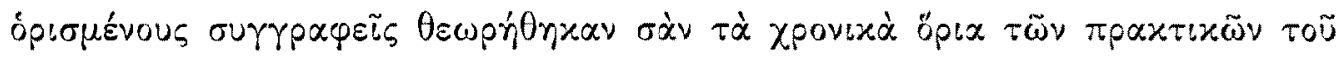

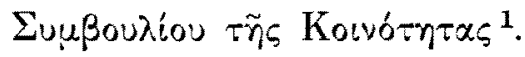

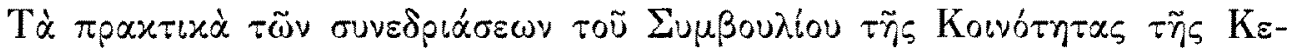

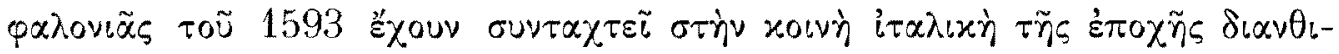

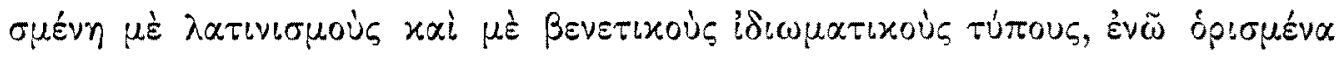

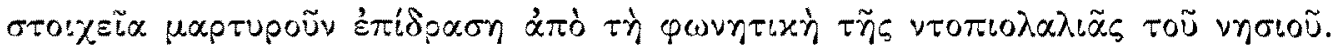

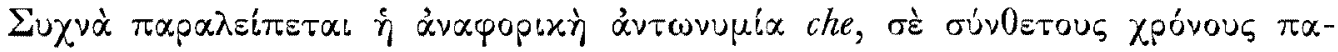

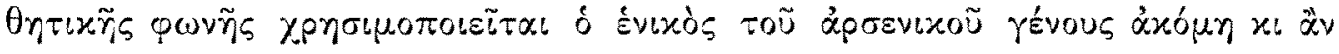

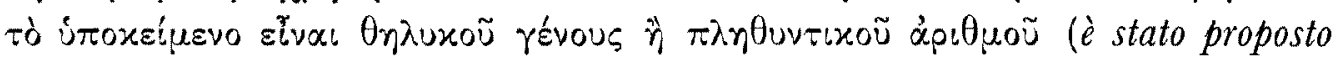
l' infrascritta deliberatione, fu rappresentato et letto l' infrascritti capitoli $\left.x . \dot{\alpha}_{.}\right)$,

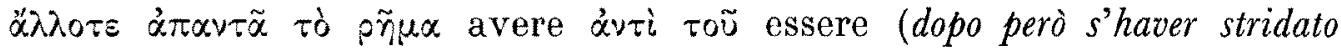

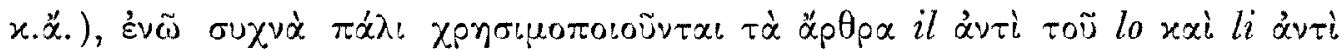

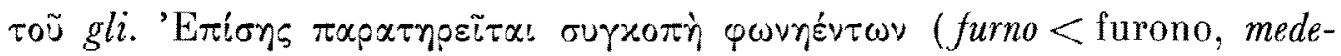

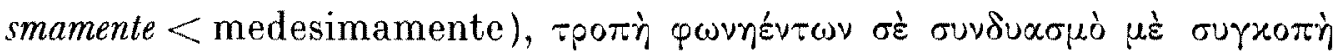

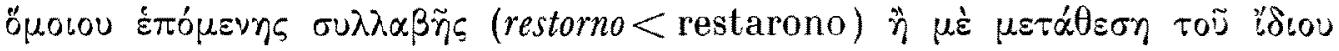

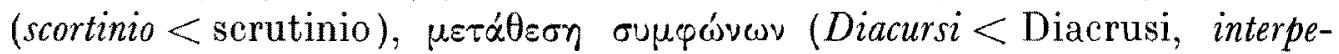
tre <interprete) $x \alpha i$ $\not \lambda \lambda \lambda \alpha \varphi \theta 0 \gamma \gamma\llcorner x \dot{\alpha}$ $\varphi \alpha\llcorner v o ́ \mu \varepsilon v \alpha$.

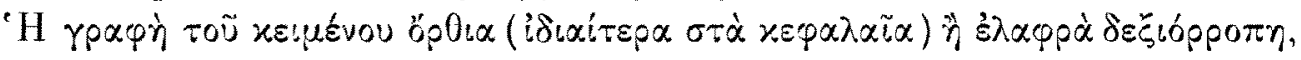

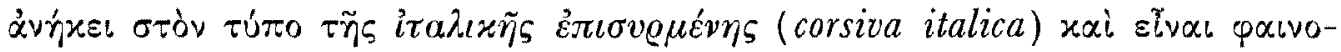

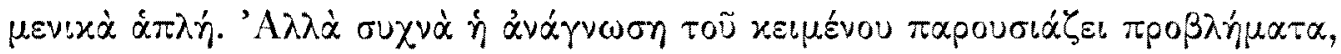

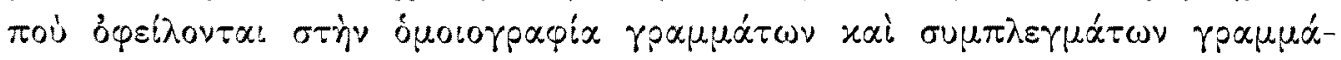

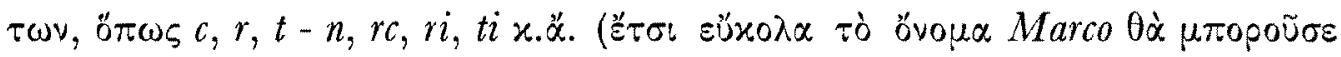

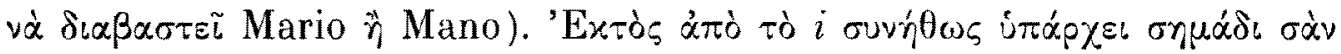

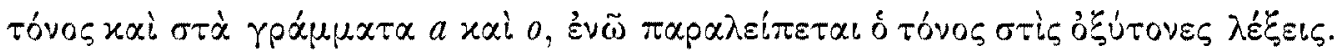

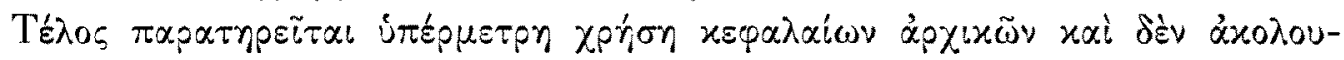

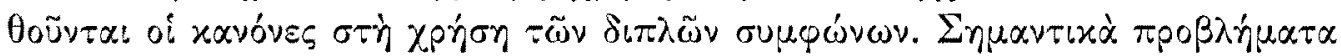

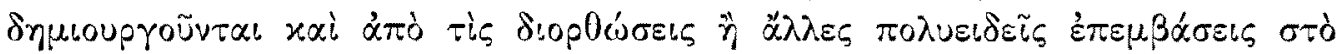

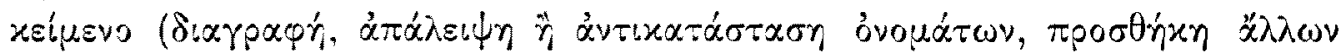

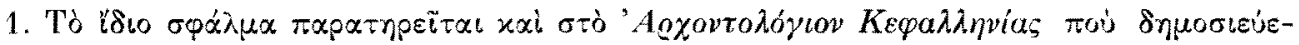

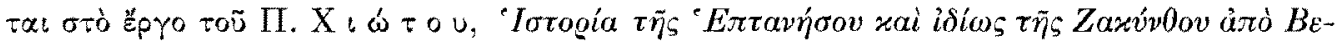

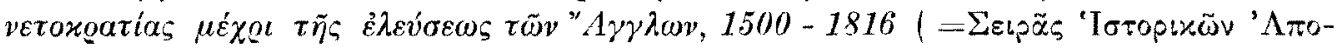

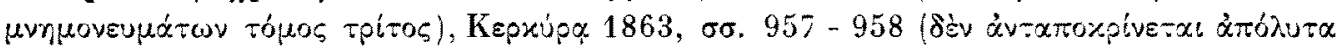

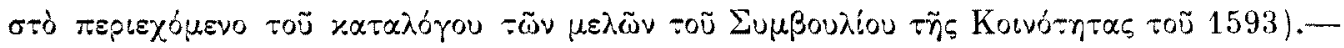

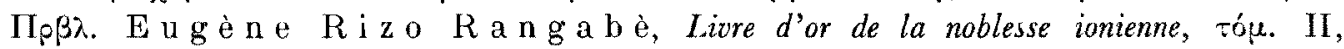

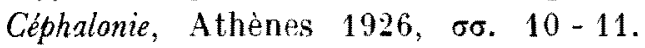




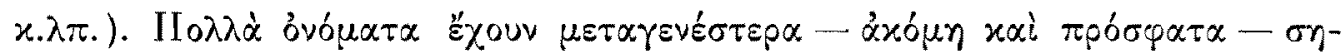
$\mu \varepsilon เ \omega \theta \varepsilon \tilde{\imath} \mu \varepsilon \dot{\varepsilon}$ ó

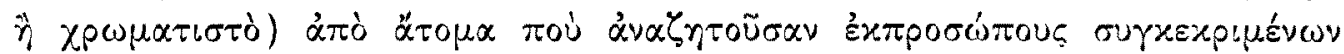

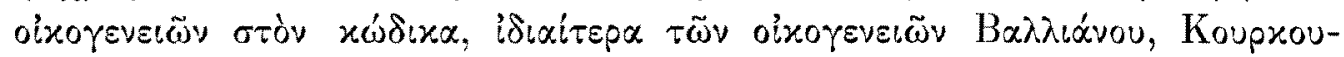

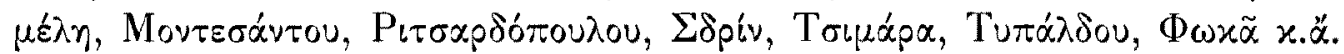

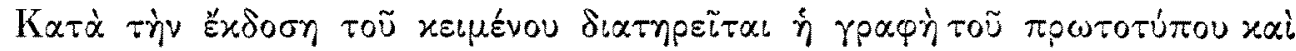

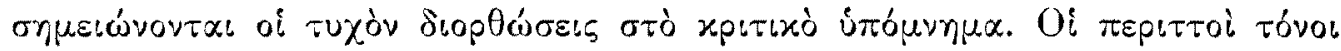

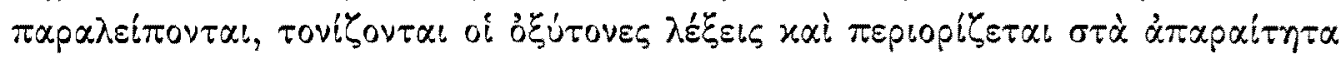

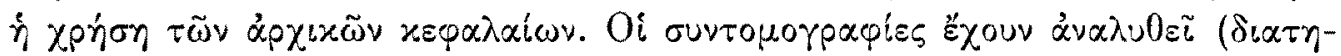

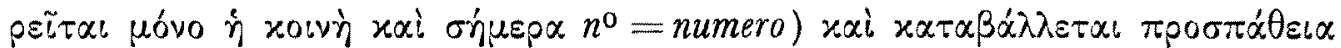

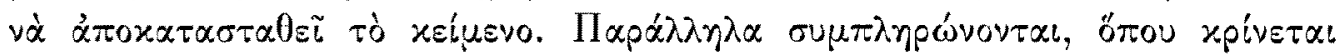

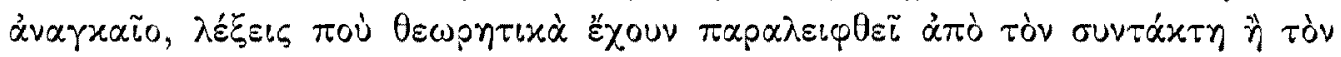

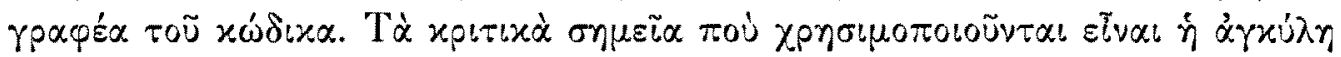

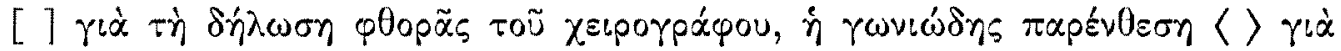

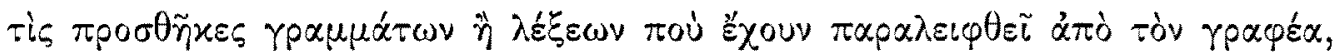

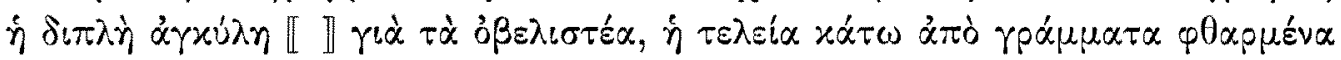

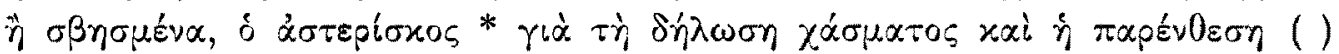

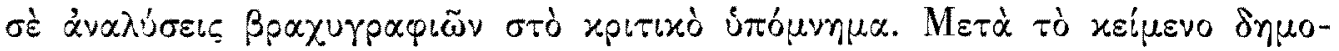

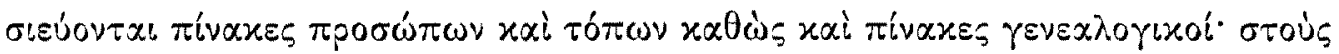

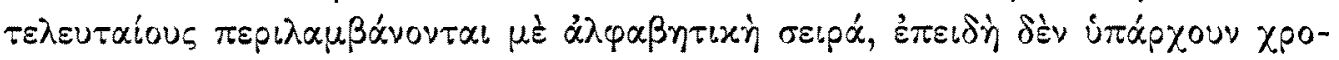

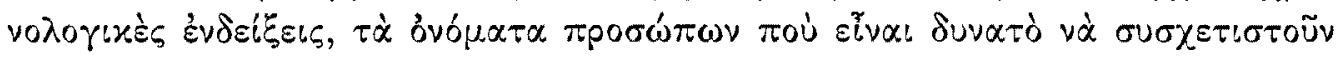

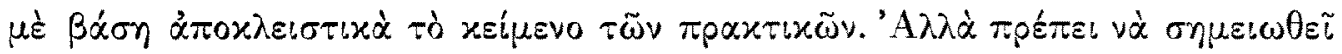

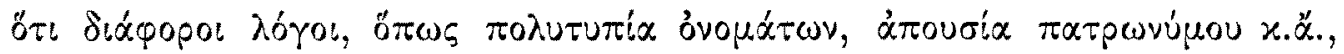

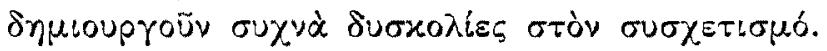

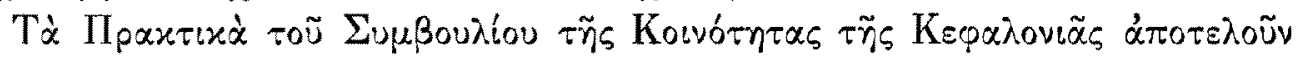

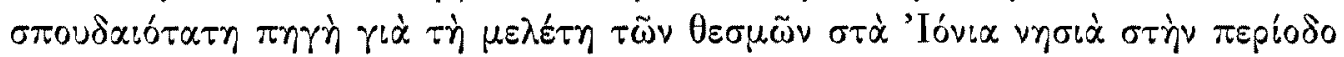

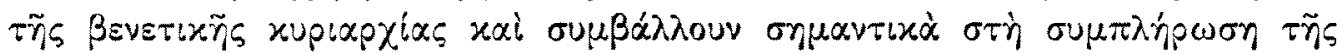

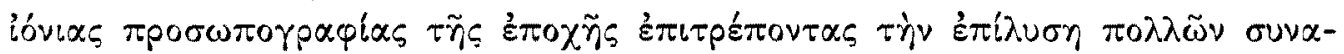

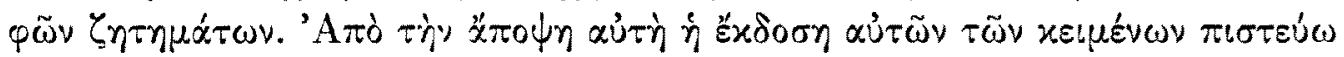

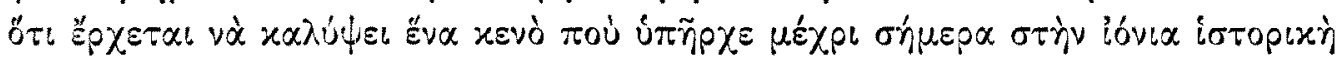
$\beta ! \beta \lambda \alpha \circ \gamma p x \varphi l \alpha$.

N. T. MOSXONAE 


\section{IN NOMINE DOMINI NOSTRI JESU CHRISTI}

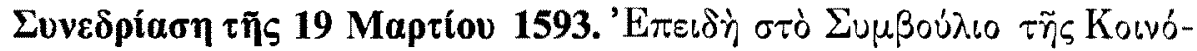

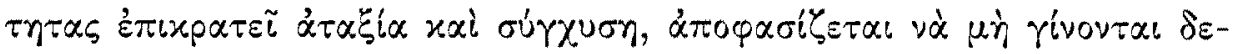

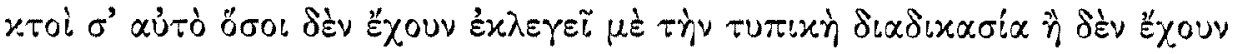

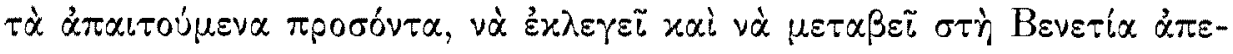

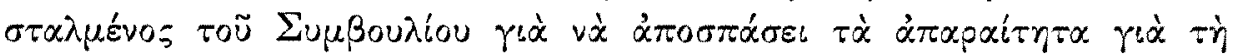

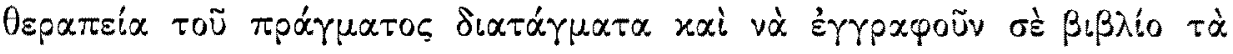

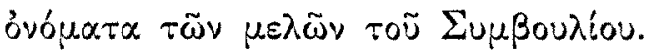

\section{Adi 19 marzo 1593}

$\varphi .1^{\mathrm{r}} \quad$ Congregato il Conseglio di questa Magnifica Communità alla presentia del Clarissimo signor Davit Bembo honorato Proveditore per venir all'in-

5 frascritta delibe[ratione] conforme all'auttorità concessa per Sua Serenità a questa Magnifica Commun[ità] proposta per li Censori ut infra:

Clarissimo signor Proveditore et Spettabile Conseglio

Mossi dal desiderio che tenimo $\langle d i\rangle$ grattifficar questo Spettabile Conseglio el Magnifica Co[mmunità], Noi Mathio Flocca de misser Andrea

10 et Dimitri Guerino cosí ricercati et a[.....] si può dire per il carico nel quale attualmente s'attroviamo di Censori d[alla] maggior parle di cittadini per li disordini ${ }^{1}\langle$ che $\rangle$ sono stati sin' hora introdu[tti] $\langle e t\rangle$ che si vanno introdducendo alla giornata in esso Conseglio, riddotto ogn'ordine [a] confusione, si come il tutto è benissimo noto, n'è parso giusto et raggi[one]-

15 vole acciò si dia compiuta essecutione a gl'ordini et a quant'è [voler] di Sua Serenità et per non manchar del debito nostro et esser imputati [di] manchamento, con licentia del Clarissimo Proveditore, consenso et parere delli sp[ettabili] Sinici, riddur il presente Conseglio et per carico di conscientia per oviar [li] disordini ${ }^{2}$ et tenir il Conseglio con quell' honorevolezza che si

20 conviene al $[$ ca]rico spetiale suo di Proveditore però:

$L^{\prime}$ anderà parte che de cetero non possa più alcuno nemine ese $[n t e]^{3}$ esser di questo Conseglio se prima fornito 〈non〉 haverà quanto riccercano $g l$ ' o[rdini], non si ponerà alla prova et fatossi ballottar, scodendo di questo Cons[eglio li] cinque sesti in tal caso s'intendi rimasto et non altrimenti;

25 alla qual [prova] non s'intendino però sottoposti li figliuoli ${ }^{4}$ de' cittadini et consiglieri origin[arii], ma fatta la leggittim' età d'anni vinti conform' a gl'ordini pos[sano] intrar senza contradittione n' obligo alcuno.

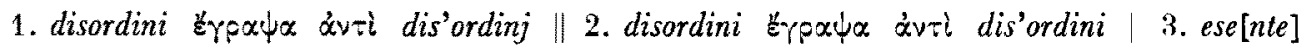

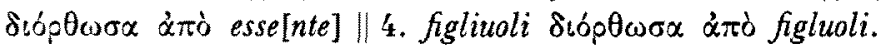




$$
\text { 1593, adi } 19 \text { marzo }
$$

Presentata alla presentia del Clarissimo signor Proveditore, ridducto il Spettabile [Conseglio della] Communità con la presentia et assenso delli magnifici signori Crist[oforo Crassà, Galeacio] Peccator et Stamaltello Ci-

5 mäa, honorati Sinici della Magnifica Com[munità], Mathio Flocca et Dimitri Guerino Censori.

$\varphi .1^{\mathrm{V}} \quad$ La qual parte balotata a bossoli et ballote tolse a pro balle numero cento $e$ vintitre et contra niuna el fu presa a tutto Conseglio.

In esso Conseglio fu medesmamente proposto per l'istessi spettabili Censori

10 l'infrascritta parte:

Che devendossi appresso quanto è stato terminato et preso per questo spettabile Conseglio proveder ad ogni incoveniente che potesse nascere, si come al più delle volte s' ha veduto succedere, sia però per ovviar ad ogni mala introduttione et $\mathrm{ch}^{\prime}$ il ${ }^{1}$ tutto passi regolatamente con le debite vie preso,

15 terminato et creato per questo Conseglio un nontio con auttorità di comparer alli piedi di Sua Serenità, o avanti qual si voglia Eccellentissimo Conseglio, Colleggio, Magistrato iusdicente et offitio d'essa Inclita Città a dimandar et elevar tutte quelle cose, lettere et altro che sono necessarie per rimediar a tali incovenienti et conservar in stato debito il Conseglio.

20 Balotata la soprascritta parte hebbe a pro balle cento et vintidue et contra una et fu presa.

Similmente in esso Conseglio per li prefatti Censori è stato proposto ${ }^{2}$, l'infrascritta deliberatione:

Che per conservar quel tanto è stato preso per l'oltrascritta parte in ma-

25 teria ch' alcuno non possa più esser di Conseglio $s[e]$ non scoderà li cinque sesti et come in essa sia preso, statuito et terminato, accioché alcuno non possi aleggar esser del numero d'esso, che tutti quelli sono del Conseglio veramente et effettualmente conosciuti et non altrimenti, però debbano in termine di giorni otto ${ }^{3}$ darsi in notta alli Censori, accioché siano descritti

30 in questo libro intitolatto numerario, non si potendo spirato il termine nottar più alcuno, ne por mano in questo libro, se non ${ }^{\mathbf{4}}$ uno di Cancelieri della Communità sottoscritto dalli tre Sindici et dui Censori; altrimenti ogni nota che fosse fatta s'intenda de niun valor; et questo spirato che sarà il presente Reggimento nel quale habbia Marco Crasso canceliero attuale

35 il carico et non altri, però il tutto sottoscritto dal Clarissimo signor Prove$\varphi .2^{\mathrm{r}}$ ditore et tre Sinici attuali. Et spirato $\|$ il soprascritto termine et non si dando

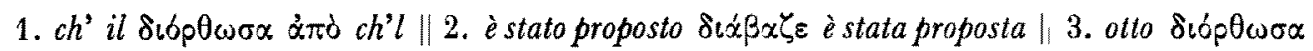

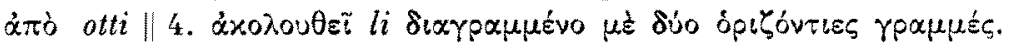


in notta come di sopra s'intendino caduti dal Conseglio et mai per alcun tempo non possano piiu esser, se non con li cinque sesti.

Balotata ${ }^{1}$ la soprascritta parte tolse a pro balle cento et vintidò et contra una, et fu presa.

5 Davit Bembo Proveditor

Christophoro Crassà Sindico

Galeacio Peccator Sindico

Stamati Cymera Scindico della Comunità

\section{Marco Crasso Canceliero}

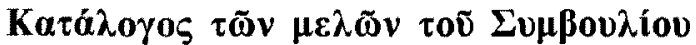

$$
\text { Numerario del Conseglio tenuto per il spettabile }
$$
misser Demetrio Guerino Censor giusta la parte et da quello copiato.

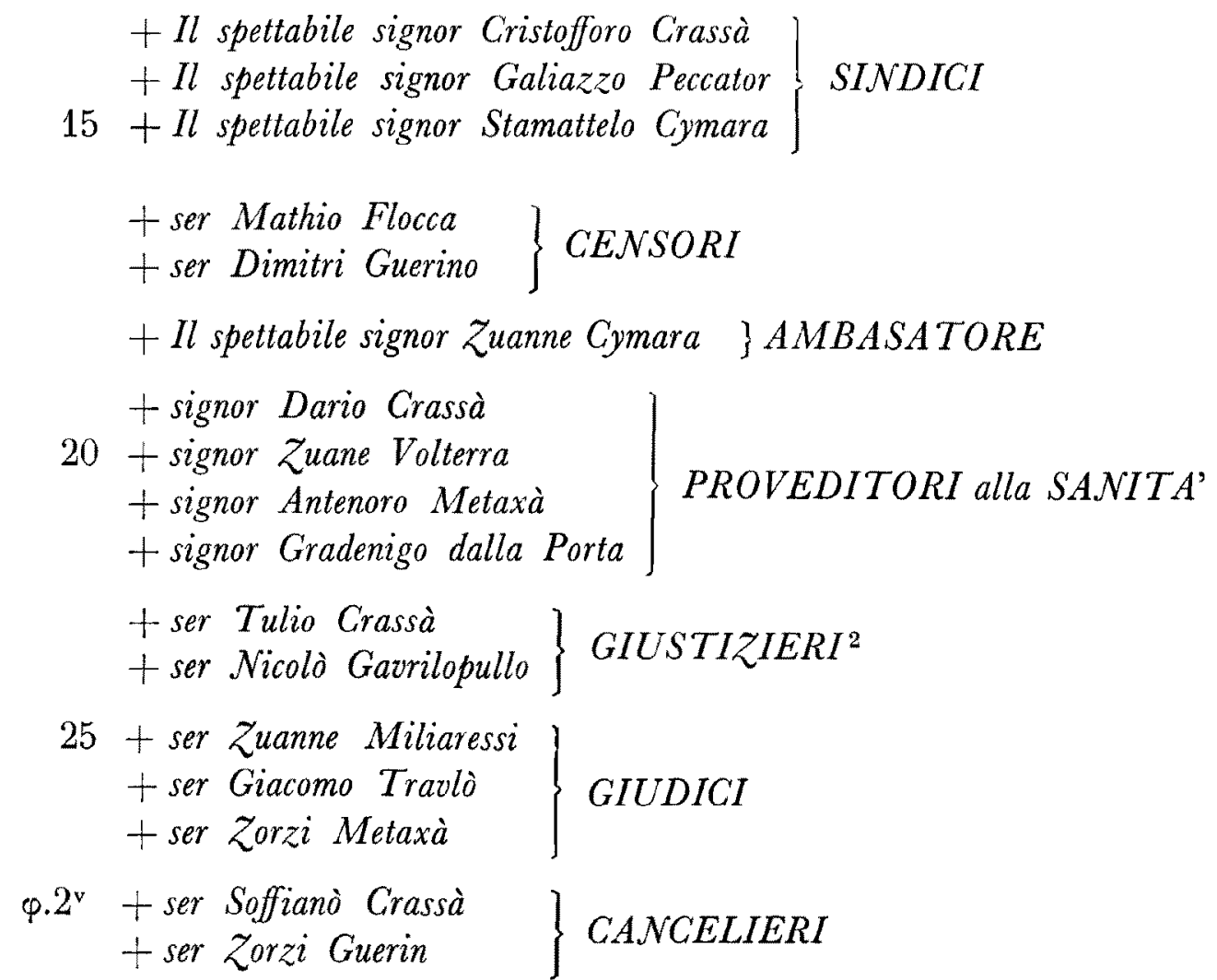




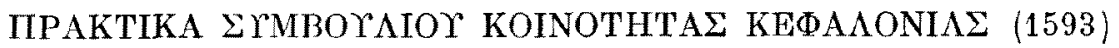

+ ser Nicolò Travlò \} CAPITANO al THEACHI

+ ser Gasparo Mingardo

+ ser Marin di Zentilini

SIGNORI alle STRADE

+ ser Zuanne Dimissiano \} COADIUTORE alli COMANDAMENTI

$5+$ Il magnifico domino Teodoro Cladà Governatore.

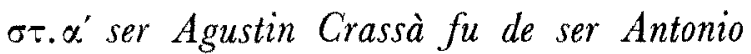

ser Zuane Crassà fu de ser Francesco

ser Alessandro Crassà fu de ser Giacomo

10 ser Steffanin Crassà $\}$ fu de ser Francesco

$\left.\begin{array}{l}\text { ser Fioravanti Crassà } \\ \text { ser Stamattello Crassà }\end{array}\right\}$ de ser Agustin

ser Draco Crassà

ser Francesco Crassà $\}$ de ser Dario

15 ser Antonio Crassà fu de ser Fulio

ser Francesco Crassà fu de ser Florio

ser Dimitri Crassà fu de ser Lucca

ser Nicolò Peccator

ser Antonio Peccator $\}$ de ser Galeazzo

20 ser Draco Comi fu de ser*

ser Antonio Valier quondam ser Zuane

ser Andrea Flocca quondam ser Mathio

ser Stamatti Volterra quondam ser Arseni

$\left.\begin{array}{l}\text { ser Zorzi } \\ \text { ser Nadal }\end{array}\right\}$ Volterra de ser Stamatti

ser Francesco Volterra fu de ser Todorin

$\left.\begin{array}{l}\text { ser Gulielmo } \\ \text { ser Nicolò }\end{array}\right\}$ Volterra quondam ser Cesare

ser Agustin Palazzuol quondam ser Piero

30 ser Francesco Palazzuo

ser Marc'Antonio Palazzuolo $\}$ quondam ser Zam' Battista

ser Mathio Salamon fu del nobilhomo ser Giacomo Antonio

ser Giacomo Strambali 


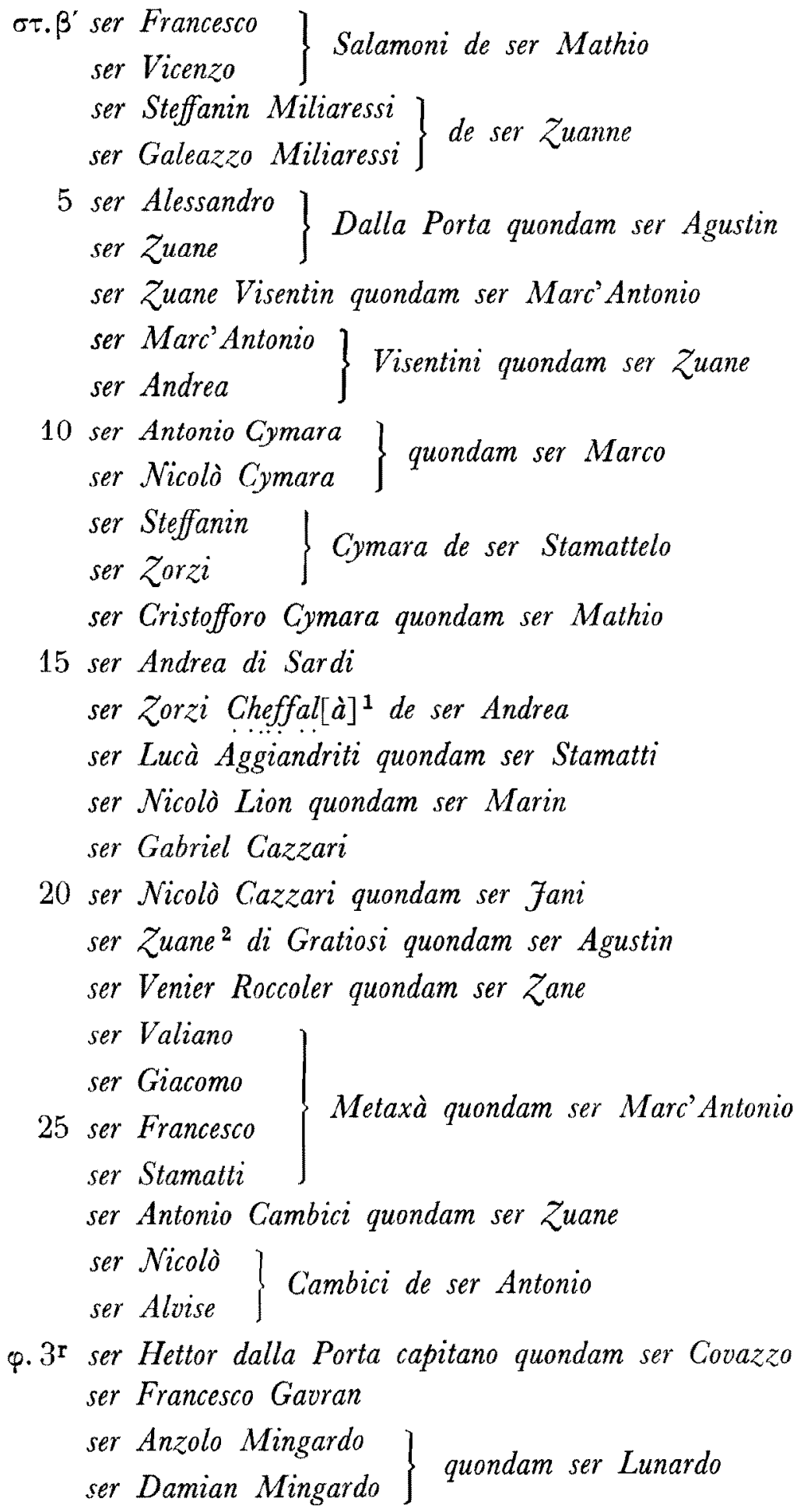

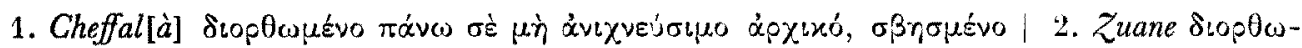

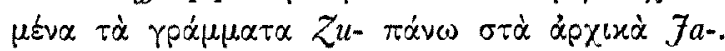




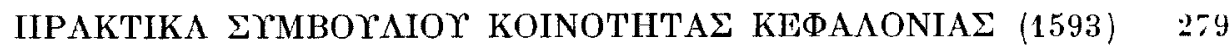

ser Manoli Fuccà fu del capitano

$\left.\begin{array}{ll}\text { ser Marcello Metaxà } \\ \text { ser Gerolimo Metaxà }\end{array}\right\}$ quondam ser Costantin

ser Zuane Sdrigna

$\left.\begin{array}{l}5 \text { ser Battista Bianco } \\ \text { ser Piero Bianco }\end{array}\right\}$ quondam ser Cosmo

ser Zorzi Asani ${ }^{1}$

ser Todorin Mingardo quondam ser Lunardo

$\left.\begin{array}{l}\text { ser Gasparo Capassà } \\ \text { ser Pavlin Capassà }\end{array}\right\}$ quondam ser Giorgilà

ser Marco

ser Hettor

Coraffà quondam ser Beno

ser Zanetto

ser Francesco Dandolo quondam ser Orlando

15 ser Orlando ${ }^{2}$ Dandolo quondam ser Andrea

ser Dimo de Nicolò scrivan delle fabriche

ser Piero Anino quondam ser Andrea

ser Ventura

ser Gerolimo $\}$ Anino de ser Piero

20 ser Nicolò

ser Francesso Milonà quondam ser Andrea

ser Costantin Milonà

ser Fabritio

ser Marco $\}$ Manessi

25 ser Giacomo Suriano

ser Odoardo ${ }^{3}$ Sdrigna quondam ser Luca

ser Lunardo Servò quondam ser Teofflo

ser Marco Cloni

ser Dome〈ne〉go da Napoli sargente delle cernide

30 ser Bortholamio Fiscardi

$\left.\begin{array}{l}\text { ser Francesco } \\ \text { ser Draco }\end{array}\right\}$ de Montesanto de papà Leo ${ }^{4}$

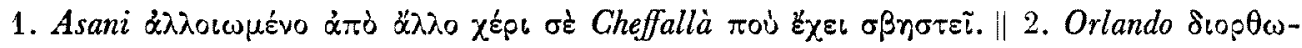

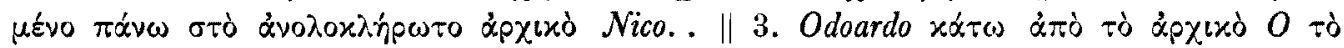

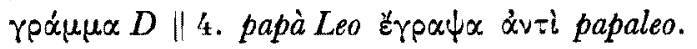




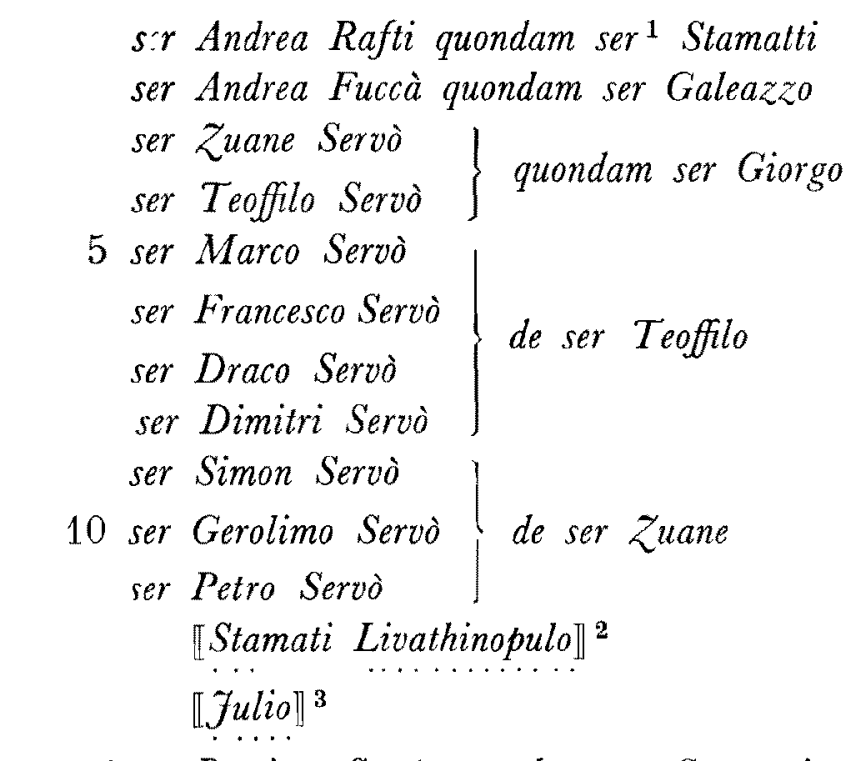

$\sigma \tau . \beta^{\prime}$ ser Battista Servò quondam ser Stamatti

15 Domino Nicolò Barbati governatore ser Pavlo Barbati capitano ser Andrea Barbati capitano ser Dimitri Lusi capitano ser Nicolò Lusi capitano

20 ser Marco Fucà capitano ser Demetrio Vinari capitano ser Apostolo Vinari capitano ser Andrea Combotecra capitano ser Benetto Combotecra capitano

25 ser Stamatti Combotecra capitano ser Petruzzo Combotecra capitano ser Frangià Lusi de ser Stamatti ser Marc'Antonio Lusi del capitano ser Lazaro Chelmi capitano

30 ser Alessandro Chelmi ser Andrea Chelmi del capitano ser Zorzi Vinari ${ }^{4}$ del capitano ${ }^{5}$

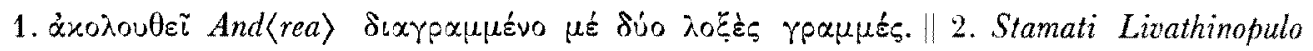

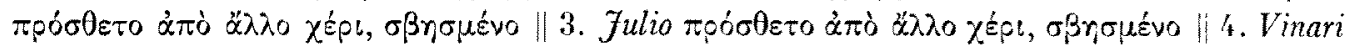

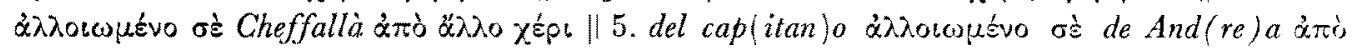
$\not 2 \lambda \lambda 0 \chi \varepsilon \hat{p}$ 
ser Pavlo Tipaldo quondam ser Nicolò

$\left.\begin{array}{l}\text { ser Lentulo } \\ \text { ser Draco }\end{array}\right\}$ Tipaldo de ser Pavlo

ser Dimitri

$\left.\begin{array}{l}5 \text { ser Costantin } \\ \text { ser Zor } \approx i\end{array}\right\}$ Tipaldi quondam ser Draco

ser Frangià Tipaldo quondam ser Andrea

ser Leono

ser Piero

Tipaldo de ser Lambrinò

10 ser Nicolò Tipaldo

ser Frangià Tipaldo

ser Dimitri Tipaldo quondam ser Marco

ser Zorzi Tipaldo

ser Stamatti Tipaldo

15 ser Vardaramo Tipaldo

ser Manoli Tipaldo

ser Alessandro Tipaldo

ser Tomà Tipaldo

ser Costantin Tipaldo

20 ser Andrea Tipaldo

ser Jani Tipaldo

ser Marco Tipaldo

quondam ser Arseni

ser Nicolò Tipaldo

ser Antonio Tipaldo $\}$ quondam ser Pavlo

25 ser Apostoli Tipaldo quondam ser Costantin

ser Lunardo Tipaldo quondam ser Carlo

ser Stamattelo Tipaldo quondam ser Velissario

ser Stamatti Melissinò quondam ser Giacoms

sor Francesco Lombardo cittadin

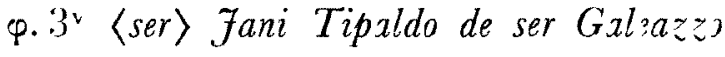

$\left.\begin{array}{c}\text { ser Lazaro } \\ \text { ser Giorgo }\end{array}\right\}$ Cambici de ser Alessandro

ser Stamati Cambici quondam papà Giorgo

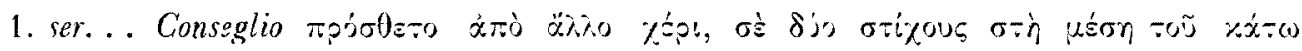
$\pi \varepsilon p \iota n$ ćplos. 
ser Zaccaria Valiano de papà

ser Apostoli Travlò quondam Federigo

ser Nicolò Cibo quondam ser Lazaro

ser Metaxà Metaxà

5 ser Francesco Rizzardopulo quondam Antonio quondam papà

ser Achilea Magulà quondam ser Franco

ser Demetrio Panà quondam ser Alvise

ser Cristofforo Carandinò quondam ser Spilioti

ser Zuane Carandinò

10 ser Stamattelo Carandinò de ser Zuane

ser Cesare Vinari quondam ser Fani $^{1}$

ser Giorgo Combotecra quondam ser Nicolò

ser Andrea Combotecra

ser Todoro Combotecra quondam ser Martin

15 ser Jani Cangadi quondam ser Sgurò

ser Hettor Combotecra del capitano

ser Draco Combotecra del capitano

ser Timothio Pandogalo quondam Manoli

ser Antonio Fuccà quondam ser Frangià

20 ser Steffanin Fuccà

ser Cristodulo Fuccà

ser Frangià Fuccà

de ser Antonio

ser Todossi Fuccà

ser Steffanin

25 ser Hettor

ser Gabriel

Fucà del capitano Lunardo

ser Anastassi

ser Orlando

ser Zuane

30 ser Steffanin

Fuccà quondam ser Hettor

ser Andrea Fuccà del protopapa

ser Galeazzo Fuccà del capitano Marco

ser Galeazzo Fuccà de ser Andrea

ser Mathio Marcopulo

35 ser Petro Metaxà

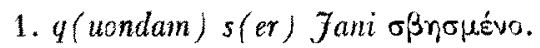




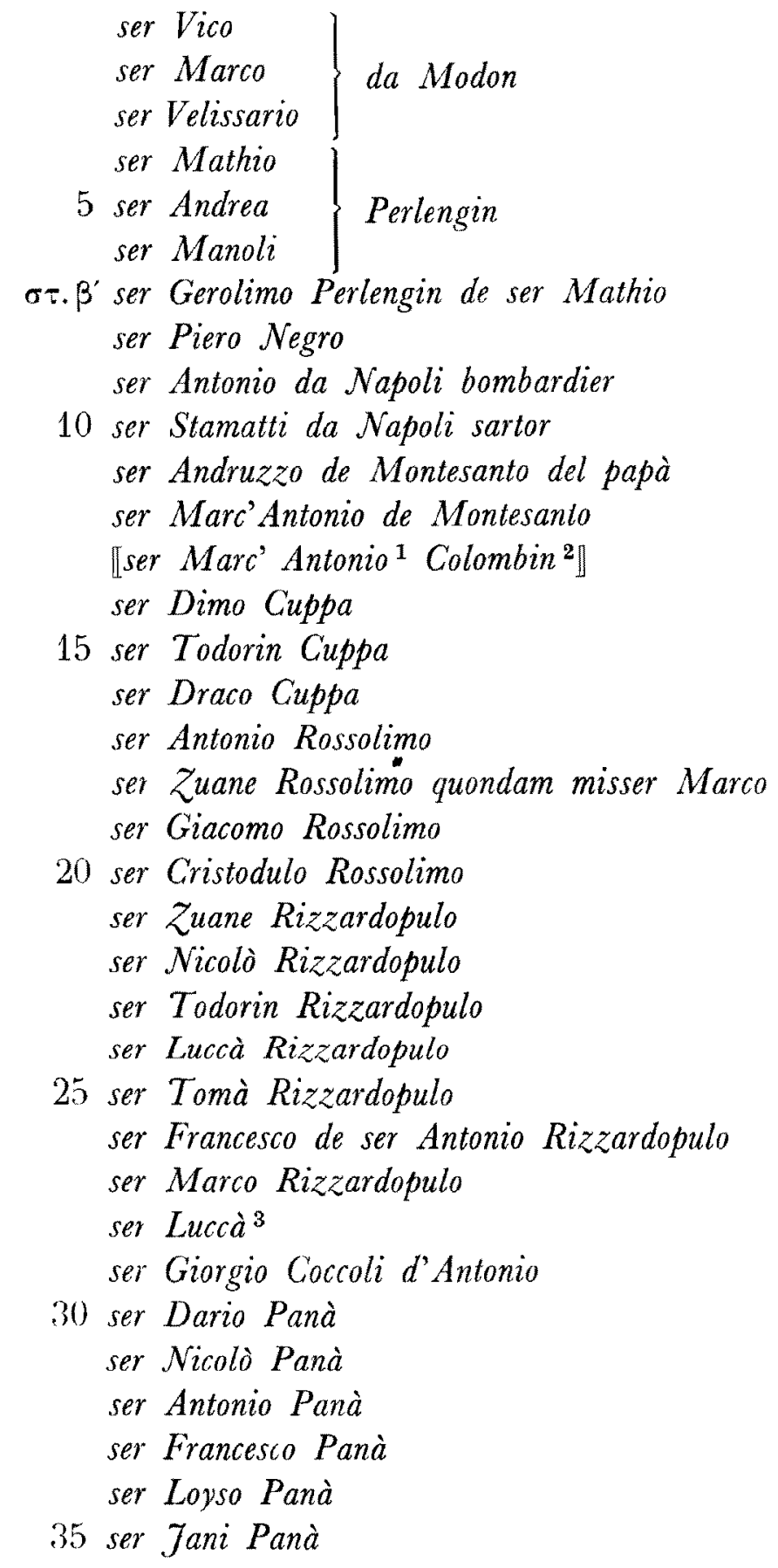

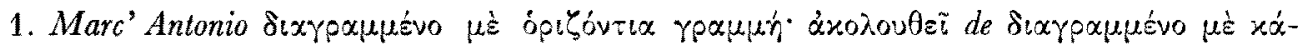

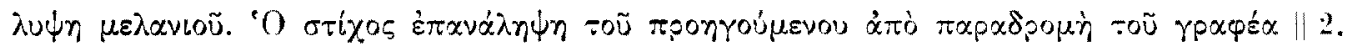

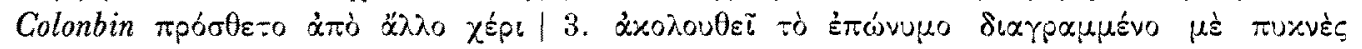

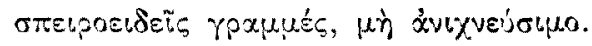




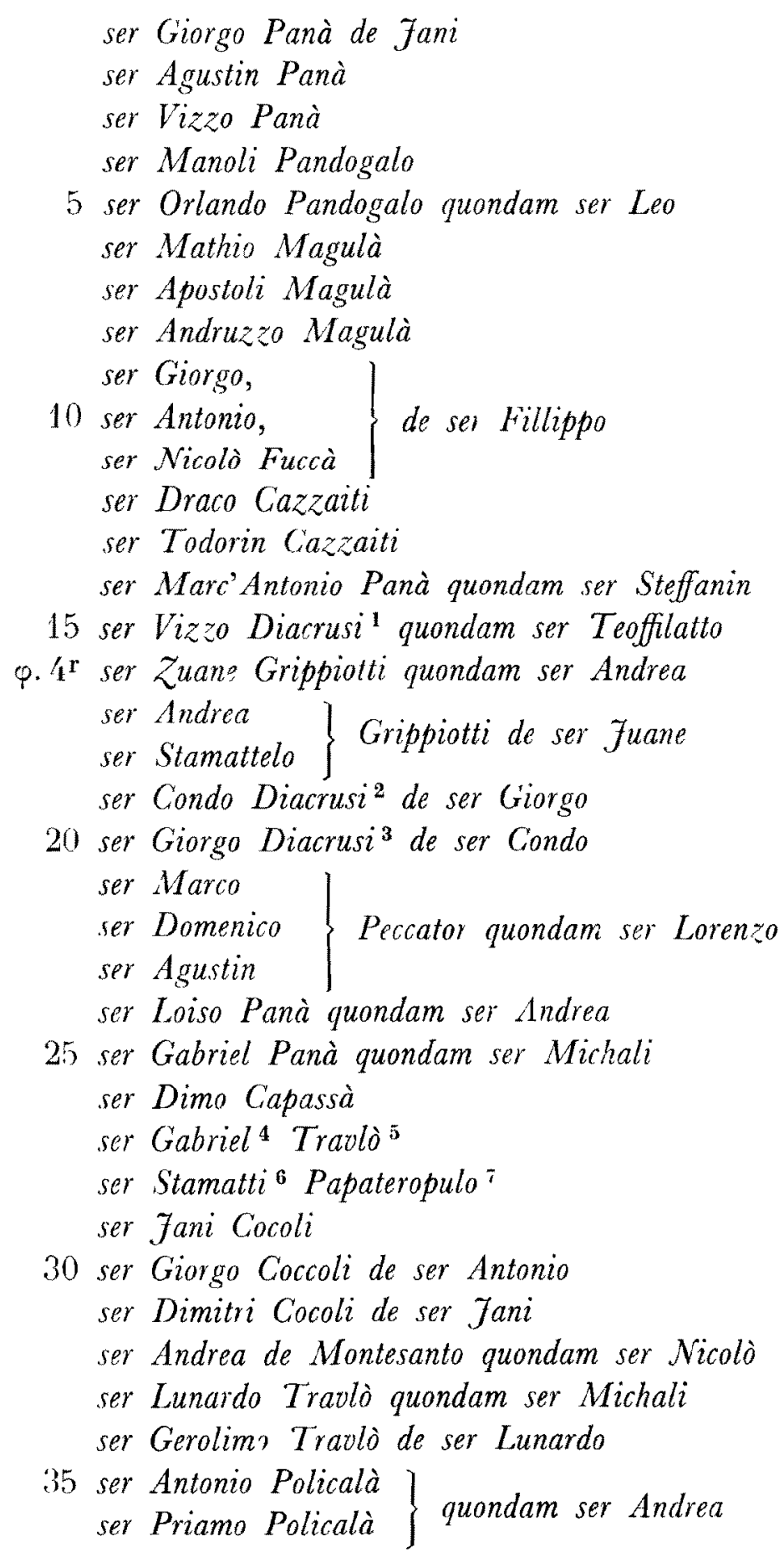

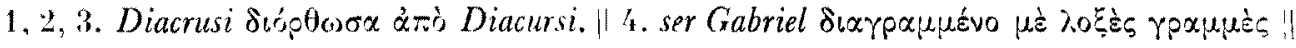

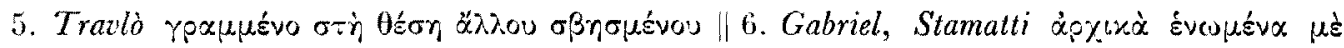

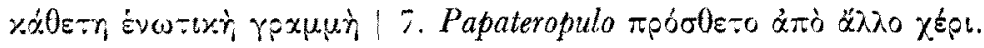


ser Giorgo Policalà

ser Nicolò Policalà de ser Giorgo

ser Zanetto de Montesanto

ser Alessandro Marcopulo quondam ser Januzzo

5 ser Zuare Marchetto de papà ser Filippo

ser Zorzi Travlò de ser Lucà

ser Jani de Montesanto de protopapa

ser Steffanin de Montesanto quondam misser Stamatti

ser Tomaso Travlò

10 ser Andruzzo Travlò de ser Stamatti

ser Zuane de Montesanto quondam ser Nicolò

ser Michali Marchetto de ser Giacomo

ser Nicolò Travlò ${ }^{1}$ de ser Leo

ser Nicolò Fuccà quondam ser Pasco

15 ser Alessandro Marcopulo ${ }^{2}$ de ser Manoli

ser Gerolimo de Montesanto del protopapa

$\left.\begin{array}{l}\text { ser Nicolò de Montesanto } \\ \text { ser Antonio de Montesanto }\end{array}\right\}$ quondam ser Apostoli

ser Zaccaria de Montesanto quondam ser Vicenzo

20 ser Michali Marcopulo quondam ser Giorgi ${ }^{3}$

ser Andrea Capassà quondam ser Michali

ser Giorgo Suli quondam ser Jani

ser Zorzi Metaxà quondam ser Lucà

ser Oratio Travlò del papà

25 ser Nicolò Valiano quondam ser Zaccaria

ser Bernardin Trechi capitano

$\sigma \tau . \beta^{\prime}$ ser Colela

ser Anzolo

30 ser Zorzi

Valsamachi quondam ser Piero

ser Marc'Antonio

ser Zanico

ser Zorzi

ser Zuane Schiadà de ser Petro

35 ser Costantin Vergotin quondam ser Vergotin

ser Antonio Janulato

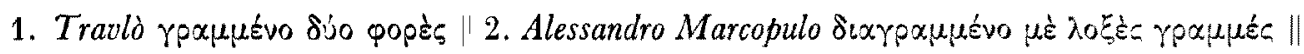

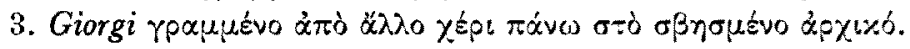


ser Nadal Rizzardopulo quondam ser Costantin

ser Antonelo de Montesanto

ser Giacomo Travlò quondam ser Michali

ser Alessandro Traulò quondam ser Manoli

5 ser Andruzzo Valiano quondam papà ser Giorgo

ser Marco Travlò de ser Zuane

ser Andruzzo Travlò de ser Nicolò

ser Barbarigo Travlò quondam ${ }^{1}$ ser Manoli

ser Zaccaria Valians de ser Antonio

10 ser Pavllo Rizzardopulo ${ }^{2}$ quondam ser Janu₹o ${ }^{3}$

ser Giorgo Travlò quondam ser Apostoli

ser Zuane de Montesanto quondam ser Stamati

ser Relo Rizzardopulo

ser Francesco Rizzardopulo $\}$ de papà ser Dominico

15 ser Andrea Pandogalo quondam ser Gabriel

ser Gabriel Pandogalo de ser Andrea

ser Bello Pandogalo quondam ser Gabriel

ser Lazarin Grippiolti de ser Zuane

ser Marc' Antonio Mavrichi quondam ser Stamatti

20 ser Stamati ${ }^{\mathbf{4}}$ Marrichi ${ }^{\mathbf{5}}$ quondam ser Dimo

ser Manoli Metaxà quondam ser Lucà

ser Draco Fuccà quondam ser Alessandro

ser Piero Coccoli quondam ser Nicolò

ser Jani

25 ser Zuane $\}$ Cocoli quondam ser Nicolò

ser Nicolò Cocoli quondam ser Stamatti

ser Battista de Montesants quondam ser Marco

ser Alessandro Peccator quondam ser Nicolò

ser Teoffilo Michalitiano

30 ser Anastassi Michalitiano

ser Massiminiano Coraffà quondam ser Todorin

ser Todorin Castellano quondam ser Antonio

ser Antonio Castellano de ser Nicolò

$\varphi .4^{\mathrm{v}}$ ser Dimo Lefchochilo quondam ser Piero

35 ser Antonio Moro

ser Pavlo Abramo

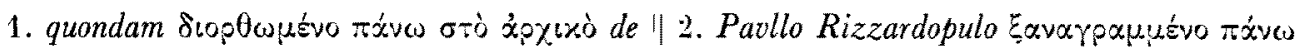

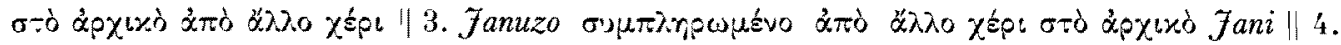

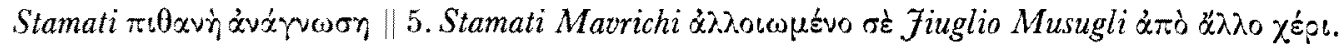




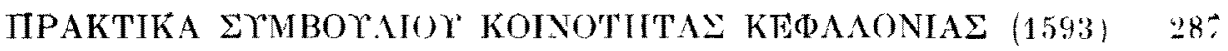

ser Zorzi Cochini

ser Costantin Caruso

ser Marc'Antonio Caruso

ser Teochari Caruso

5 ser Zuane Caruso

ser Anzolo Caruso

ser Francesco Caruso

ser Nicolò Caruso

ser Todorin Caruso

10 ser Gerolimo Caruso

ser Polo Cibo

ser Teofflo Cibo

ser Scipion Miliaressi quondam ser Nicolò

ser Mathio della Bionda quondam ser Antonello ${ }^{1}$

15 ser Zuane Marcopullo quondam ser Steffanin

ser Dimo Valiano quondam papà ser Marco

ser Benetto Stoppa bombardier

$\left.\begin{array}{l}\text { ser Nicolò } \\ \text { ser Marco }\end{array}\right\}$ Marchetto de ser Giacomo

20 ser Zaccaria

ser Dimo

ser Costantin

ser Apostoli

ser Giorgo Cambici de ser Alvise

25 ser Battista

ser Elia

Valiani quondam papà ser Marco

ser Marino

ser Zuane Policalà de ser Giorgo

ser Marc'Antonio de ser Giorgo Antippa

30 ser Simon

ser Nicolò

ser Piero

ser Michel

ser Jani Rizzardopulo de ${ }^{2}$ ser Andrea

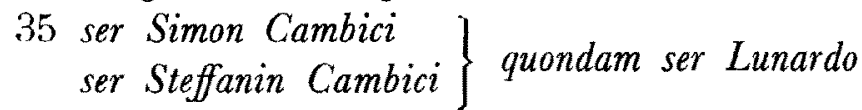

Rizzardopuli quondam Jani

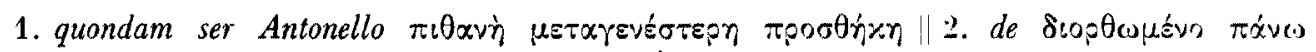
rávw oтò ג̇pxเxò $q$ (uondam). 


\author{
ser Anastassi Rizzardopulo quondam papà \\ ser Dimo Rizzardopulo quondam ser Stamatti \\ ser Anastassi Magulà 2 \\ ser Nicolò Magulà quondam ser Franco \\ 5 ser Zuane Marcopulo ${ }^{3}$ quondam papà \\ ser Alvise Flatro \\ $\llbracket$ ser Stamatti $\|^{4}$ \\ бт. $\beta^{\prime}$ ser Calogero Marcopulo quondam ser Steffanin \\ ser Nicolò Englesi quondam ser Jani \\ 10 ser Cristofforo Valiano quondam ser Antonio \\ ser Lorenzo Salamon \\ ser Zorzi \\ ser Velissario \\ ser Tiberio \\ Salamoni \\ 15 ser Sebastian \\ ser Alvise \\ ser Benetto Sicilian \\ ser Gerolimo Sicilian 5 \\ ser Dimitri da Paris bombardier et spader \\ 20 ser Zorzi Panà de ser Alvise \\ ser Giorgo Panà de ser Marco \\ ser Lunardo Panà de ser Francesco \\ ser Piero Panà de ser Todorin \\ ser Stati Metaxà de papà Alvise non è in età \\ 25 ser Lorenzo \\ ser Giorgo \\ ser Agnandio ${ }^{6}$ \\ $\langle$ Metaxà?〉 in pupilar età \\ ser Achile Rizzardopulo de ser Costantin \\ ser Stamattelo Zombo \\ 30 ser Pavlo Marcopulo quondam papà ser Jani \\ ser Dimn Cardachi
}

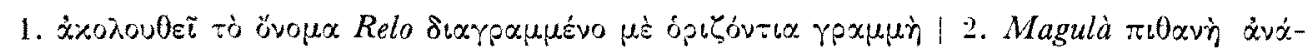

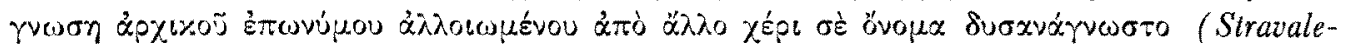

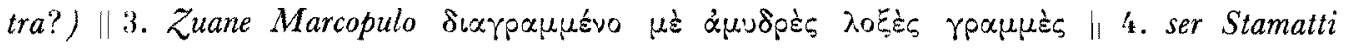

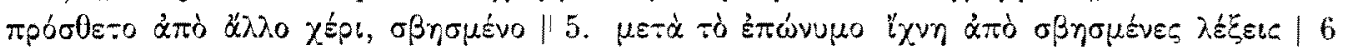

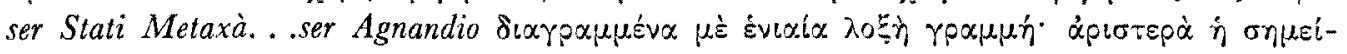
$\omega \sigma \eta$ De numero senza pregiuditio a tempo debito. 


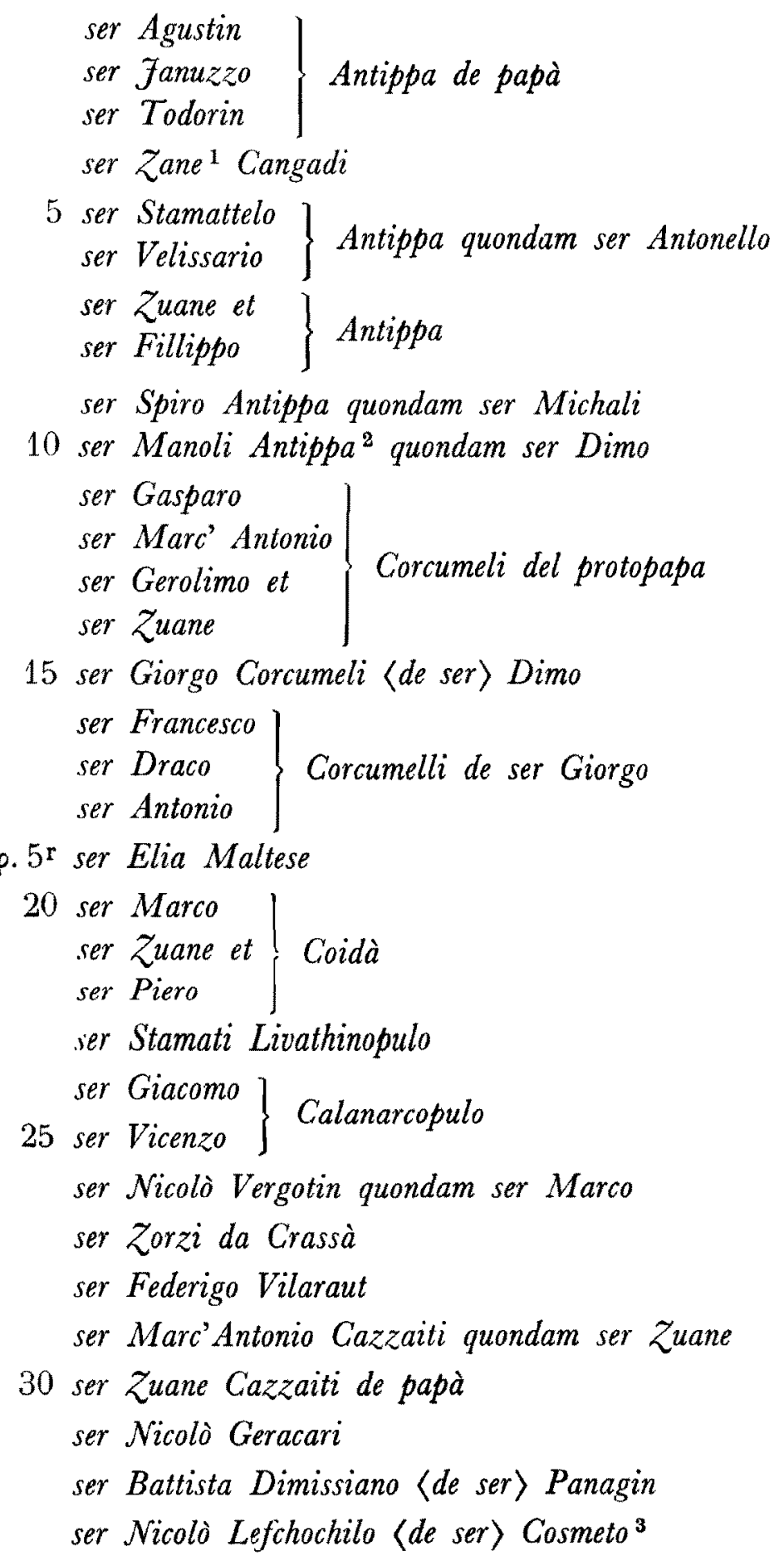

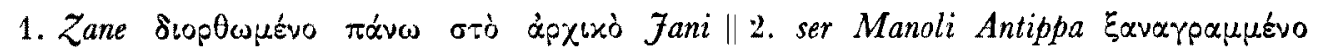

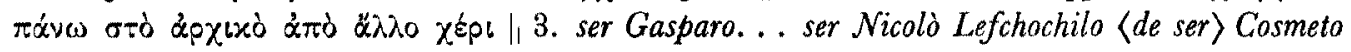

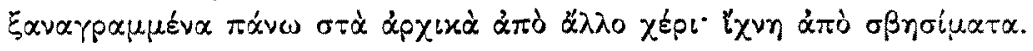


ser Steffanin Tipaldo quondam ser Giacomo

ser Velissario Tipaldo quondam Petro ${ }^{1}$ deto Bellissario ${ }^{2}$

$\llbracket s e r \rrbracket^{3}$

$\llbracket s e r \rrbracket^{4}$

$5 \llbracket \llbracket \operatorname{ser} \rrbracket^{5}$

$\llbracket \operatorname{ser} \rrbracket^{6}$

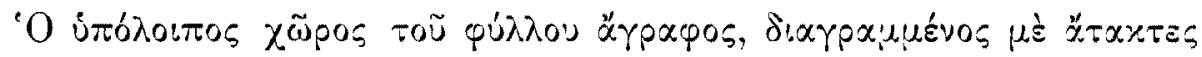

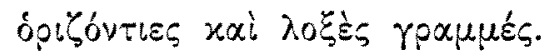

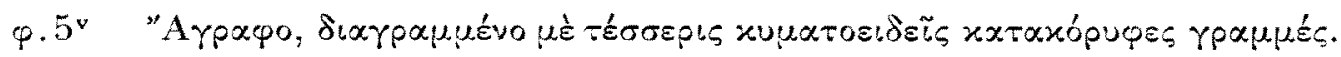

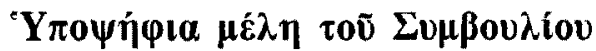

$\varphi \cdot 6^{\mathbf{r}}$

NOMI DI QUELLI

$\langle$ che $\rangle$ deveno esser balotati con li cinque sesti giusta la parte

$10\langle$ ser $\rangle$ Stathi $\langle$ di ? $\rangle$ Lefteri

$\langle$ ser $\rangle$ Jani Crisovergi

$\langle$ ser Nuzzo Mistro Callò

〈ser〉 Giorgo Mistro Callò de ser Nuzzo

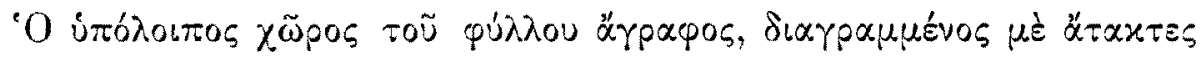

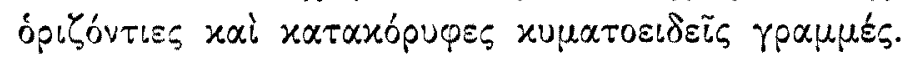

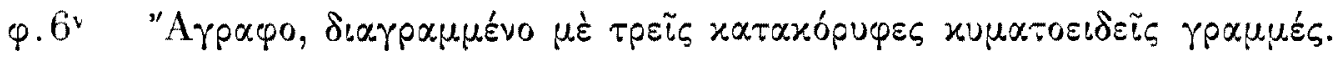

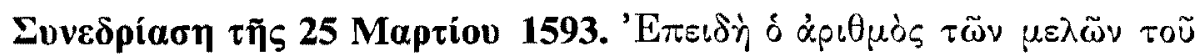

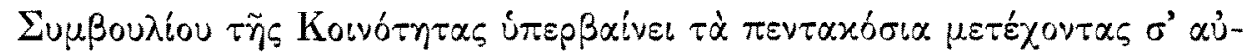

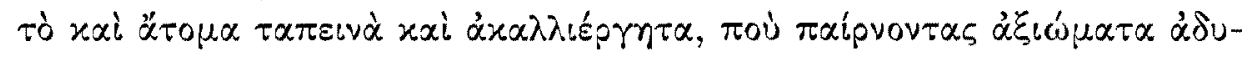

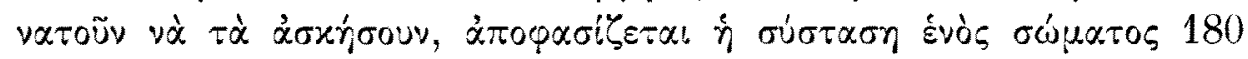

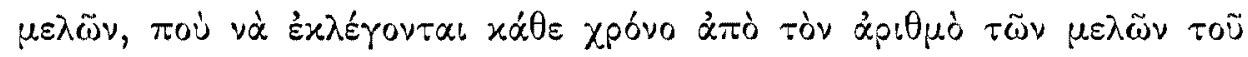

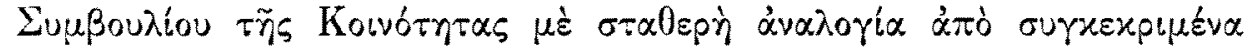

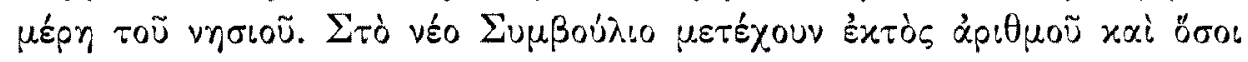

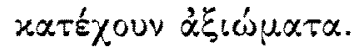

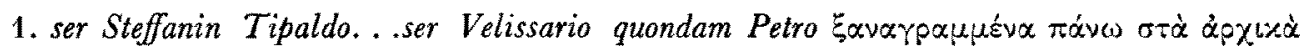

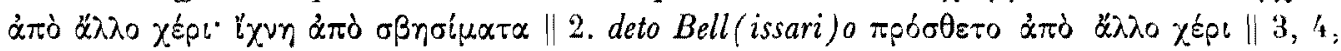

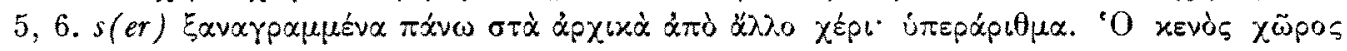

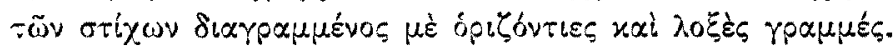


Congregato il Conseglio di questa Magnifica Communità al numero di cento e disdotto alla presentia del Clarissimo signor Davit Bembo dignissimo Proveditore, premesso il suon della campanna ordinaria per venir all'in-

5 frascritta deliberatione proposta per li spettabili Sinici et misser Demetrio Guerrino Censor ut infra, videlicet:

Havendo noi Cristofforo Crassà, Galeazzo Peccator et Stamattelo Cymara, Sinici, et Dimitri Guerino Censor, considerato di quant' importantia sia il bell' ${ }^{1}$ ordine et honorevolezza apporti a quelli che l'osservano, come

10 all'incontro il disordine ${ }^{2}$ partorisce confussione, mal effetti et pessime introduttioni, et veduto il molto disordine ${ }^{3}$ nel qual s'attrova questo Conseglio et di quanta importantia sia il rimediarvi, portando irrissione a chi s'attrova presente, si per la qualità $\langle$ di $\rangle$ persone, come per il numero tanto grande ch'ascende a cinquecento et più, essendovi stati introddutti molti, contra la

15 parte dell'Eccellentissimo Senato, d'ogni qualità, artisti, mecanici et angarizzati, di modo ch'havendo Sua Serenità concesso poter riddur consiglio di primarii, sono per questa via oltre il contravenir per diretto ad essa, conumerati fra cittadini gente bassa et che sono tenuti a fattioni personali et per questa via indiretta vengono a fuggir le dirette et giuste fattioni ${ }^{4}$ debite

20 al Principe et alla giornata va accresendo di maniera che la cittadinanza et nobiltà che in ogni locco è tanto stimata, si per la civiltà di costumi, come per il goder et fruir quell'honorevolezze che vengono dal Principe acciò datte, è vilipesa et mal stimata, per il che molte fiate occorre con pocco decoro publico, disonor di questa Magnifica Communità et danno de

25 poveri, che gente infime et del tutto rozze per favori de pari loro troppo accresciuti conseguiscono offitii et altro che non sano né puono in modo alcuno administrare con danno et malissimo essempio; però devendo per il carico che tenimo invigilar continuamente al bene, all' honorevolezza et buona administratione d'offitii et rimediar ad $u^{5}{ }^{5}$ tal disordine ridducendo le cose $\varphi .7^{\mathrm{v}}$ in buon stato $\|$ et compiuta ordinatione conoscendo massime cosi esser universal oppinione come tanti $n^{\prime}$ hanno ricercato ${ }^{6}$ più fiate, et però:

L'anderà parte che del numero descritto in essecutione della parte presa in questo Consiglio niuna discrepante de dí 19 Marzo'93 sia per questo Conseglio preso, statuito et terminato che per li descritti ut supra si possa

35 reddur il conseglio et elegger d'esso numero cento e ottanta et non più quali siano dal Castello, Borgo et locci qui sotto dichiariti, tanti per locco come

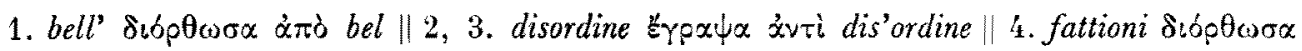

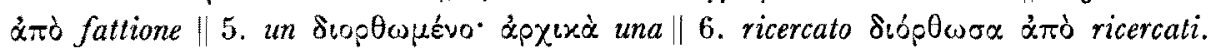


qui sotto sarano annotati, qual compartimento di numero sia per sempre dovendo tutti d'essi locci che sono delli descritli et attuali del Conseglio porsi a piacer loro alla prova et quelli che scoderano maggior numero di balle al supplimento d' esso numero s'intendano rimasti, non potendo però

5 esser delli rimasi se non tanti per ogni locco ut infra; et fatti li cento $e$ ottanta come di sopra per tempo venendo a morte, partendossi o absentandossi alcuno, la Domenica immediate seguente, doppo però s' haver stridato ${ }^{1}$ ''habbia ad elegger in locco suo dal corpo di tutti quelli che sono annotati nel presente libro, intitolato Numerario del Conseglio, di modo che continuamente resti

10 il numero di cento e ottanta et ogn'anno siano fatti altri cento e ottanta del corpo d'esso numero di quelli però che sarano eletti per balotarsi da quelli del corpo delli cento et ottanta et non altri; non patendo però gl'eletti contumatia alcuna, ma possa ogn'uno d'essi esser balotati et eletti ${ }^{2}$ ogn'anno, né possi esser nominato alcuno n'hora né giamai per esser balotatto, se non

15 sarà delli descritti che venivano in questo Conseglio, ma in caso $\mathrm{ch}^{3}{ }^{3}$ alcuno havesse oppinione di farsi nominare, debba prima farsi balottare per il Conseglio et scodendo li cinque sesti conforme alla parte de di 19 Marzo '93; scassi che gl'haverà all'hora si possa por alla prova come di sopra. Delli quali cento e ottanta non s'intendano quelli che sono attuali in offitii,

20 ma siano conumerati nel Conseglio extra 4 il corpo preditto intendendossi sempre quelli che serano in offitii rimasti del Consegtio oltr' il numero.

Dichiarando però che si debba ogn'anno avanti ogn' altro venir ad elellione de gl'offiti, nelli quali si possano balotare si gl'attuali del Conseglio com' etiam gl'altri non rimasti, però di quelli che sarano notati nel preffatto

25 numerario et siano eletti per quelli del Conseglio et poi divenir al numero $\|$

p.8r hora terminato; nelle quali balotattioni di cento e ottanta non si possa per questa volta tanto cacciar da capello se non quelli istessi che sarano balotati nelle sole loro balotationi; et gl'altr'anni s'osservi in ciò quello che si fa nell'alma cyttà di Venetia.

30 Devendo inanzi l'aprir del Conseglio, presa che sia la parte, far quell' elettione $c h$ ' $i l^{5}$ tempo presterà commodilà et susequentemente sin al compiuto numero. Oltre di ciò sia dichiarito ch' ogn' anno per l'avenire otto giorni avanti a tal elettione del Conseglio sia stridatto per inteligentia di quelli del Conseglio che si vorrano far nominare et pore alla prova accioché tutti ${ }^{6} n^{\prime}$ habbiano no-

35 tilia, intendendossi però ogn' anno et ordinariamente quelli del... ${ }^{7}$ et quelli del Castello un'istessa cosa quanto al Conseglio et non della compartita.

1. s' haver stridato $\delta\llcorner\alpha \beta \alpha \zeta \varepsilon$ essersi stridato $\mid 2$. balotati et eletti $\delta\llcorner\alpha \beta \alpha \zeta \varepsilon$ balotato el eletlo

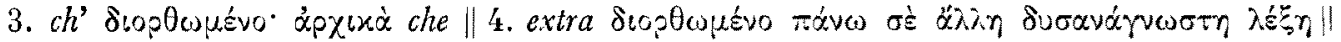

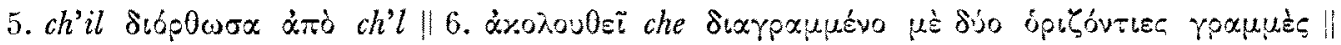

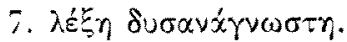




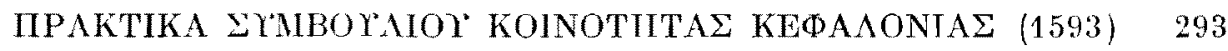

\section{Compartita}

Castello et Borgo $n^{\circ}$ sessanta dui Chieramiés $n^{0}$ vinticinque Travlata $n^{0}$ vinti

5 Talamiés $n^{0}$ disdotto

Lachitres $n^{0}$ vinti

$\left.\begin{array}{l}\text { Spartea et } \\ \text { Pesada }\end{array}\right\} n^{\circ}$ quindici

Palich et altri luoghi lontani $n^{\circ}$ vinti

$\left\{\begin{array}{l}\text { Della parte } \\ \text { dell'Eccel- } \\ \text { lentissimo } \\ \text { Senato }\end{array}\left\{\begin{array}{l}\text { Non comprenden- } \\ \text { dossi in esso } \\ \text { numero gl'attuali } \\ \text { in offittio }\end{array}\right.\right.$

10 Balotata la soprascritta parte tolse di sí cento et ${ }^{1}$ di no vintidò et fu presa.

Nomi di quelli ch' intervenero alla soprascritta balotatione

$\sigma \tau . \alpha^{\prime}$ Il Cilarissimo signor Proveditone

Il spettabile misser Cristofforo Crassà

15 Il spettabile misser Galeazzo Peccator Il spettabile misser Stamattelo Cymara

Sinici

Marco Crasso Canceliero

$$
\left.\begin{array}{rl}
\sigma \tau . \beta^{\prime} & \text { ser Mathio Flocca } \\
& \text { ser Dimitri Guerino }
\end{array}\right\} \text { Censori }
$$

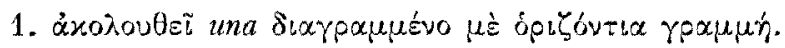




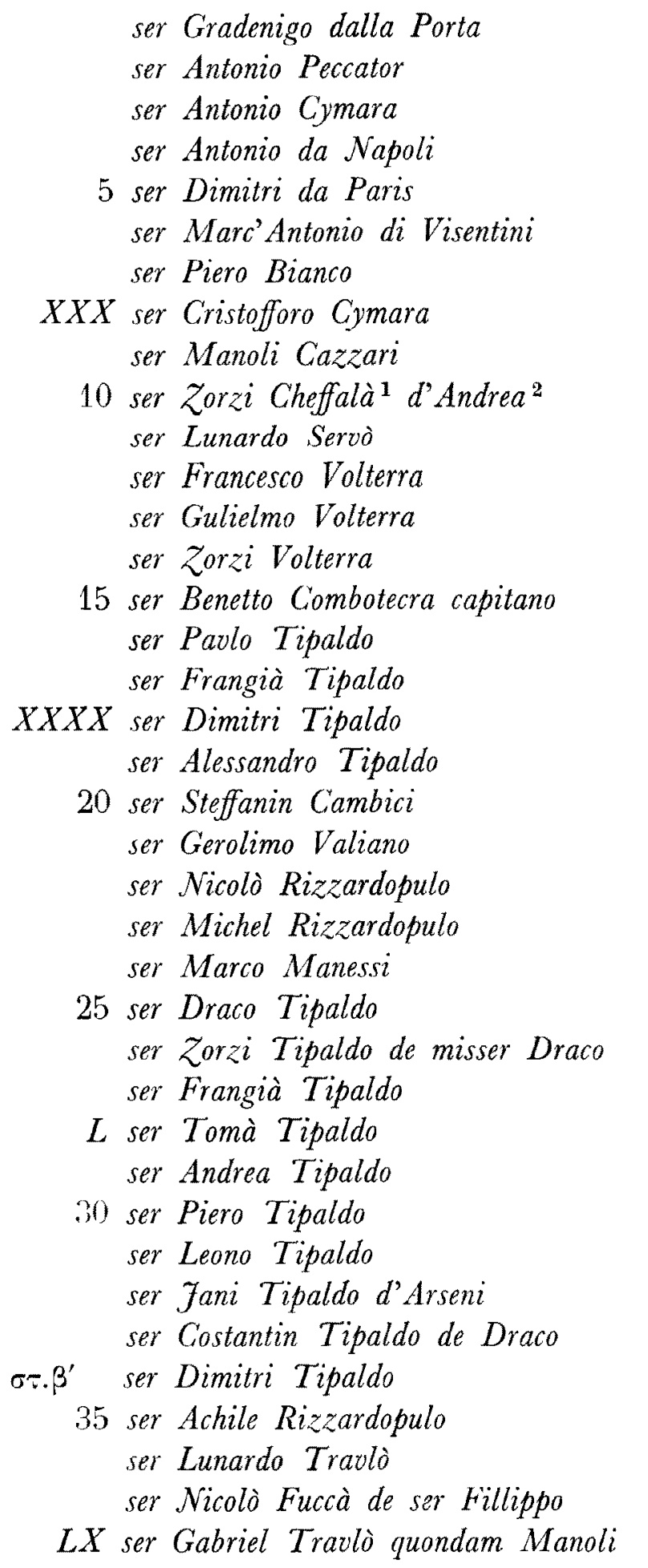

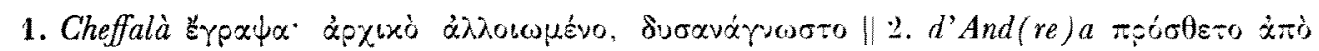

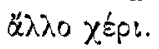




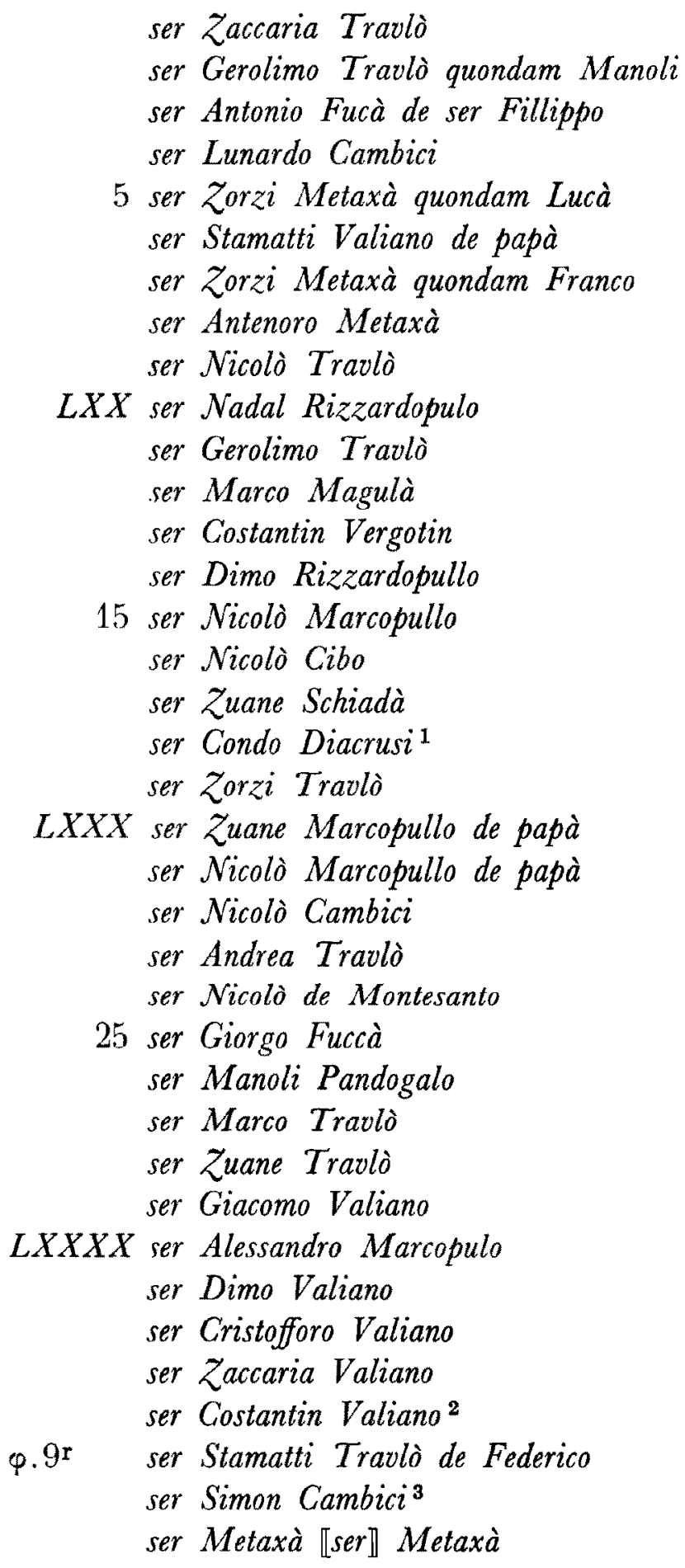

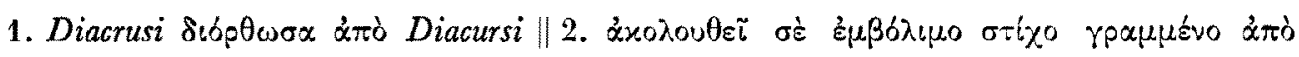

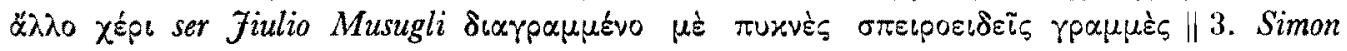

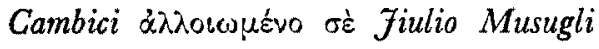




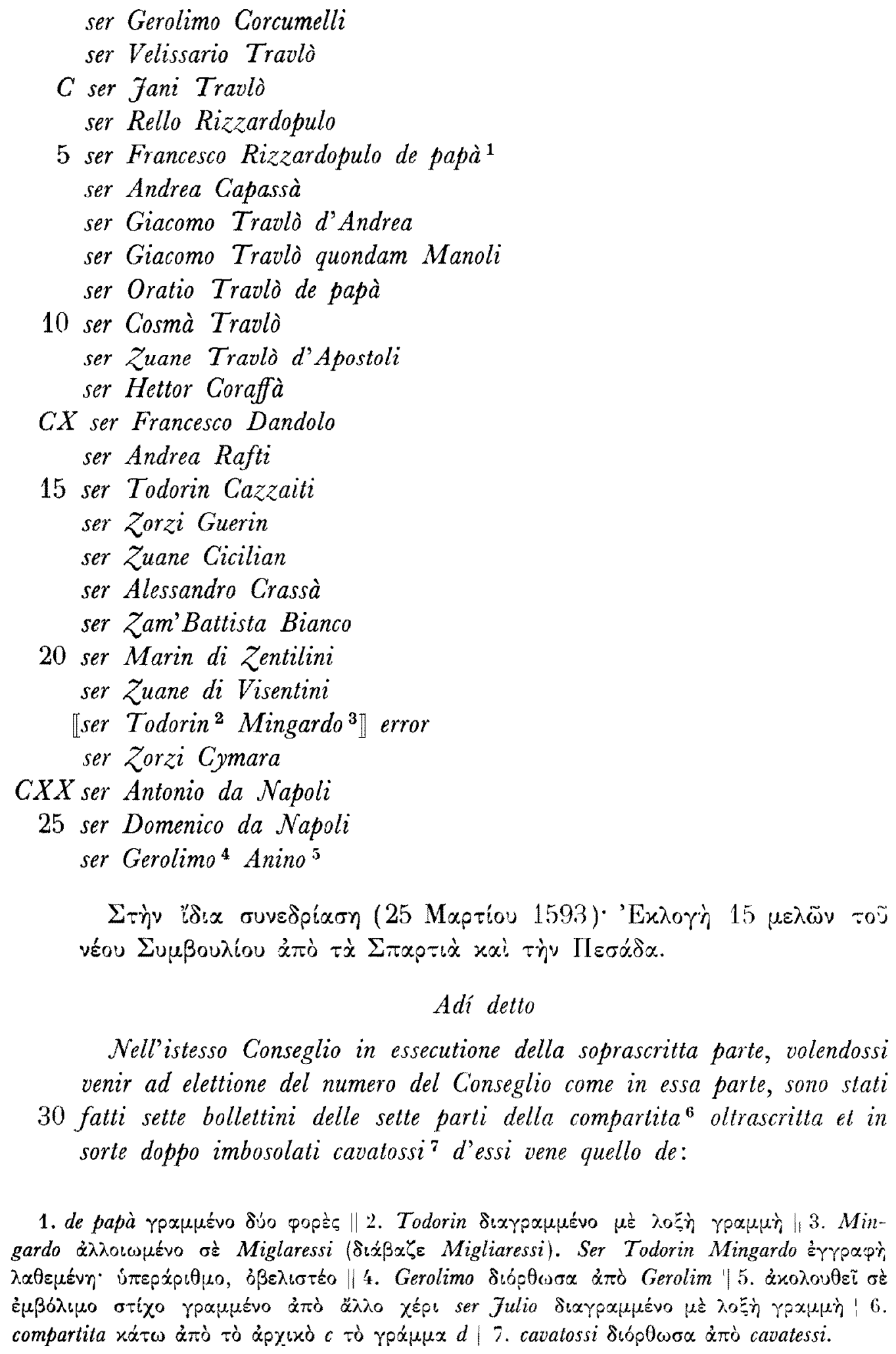

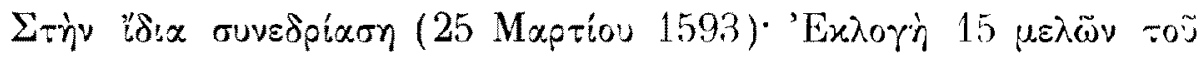

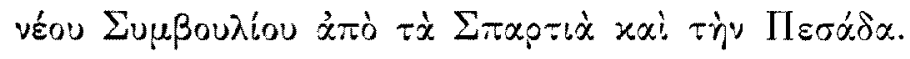

Adi detto

$\mathcal{N}$ ell'istesso Conseglio in essecutione della soprascritta parte, volendossi venir ad elettione del numero del Conseglio come in essa parte, sono stati 30 fatti sette bollettini delle sette parti della compartita ${ }^{6}$ oltrascritta et in

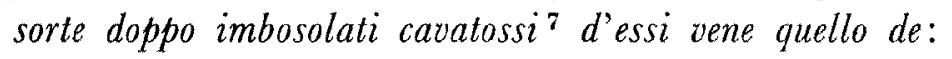

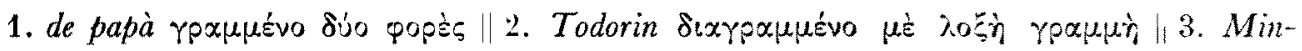

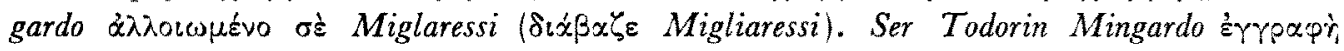

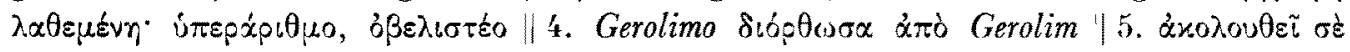

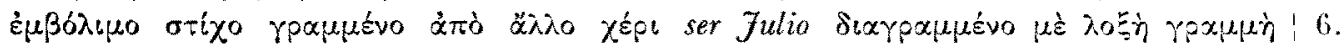

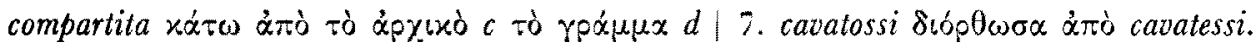




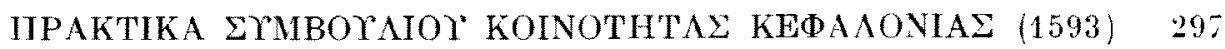

\section{SPARTEA et PESADA}

Fatto il scrotinio ${ }^{1}$ per il Conseglio sono stati dalli consiglieri eletti et o. $9^{\vee}$ nominati $\| l i$ qui sotto annotati per esser balotati nel numero; quali sono stati balotati:

5 DE SI DE NO

$\begin{array}{lll}38 & 84 & \text { ser Zorzi Valsamachi } \\ 83+ & 39 & \text { ser Anastassi Panà } \\ 80+ & 42 & \text { ser Francesco Rizzardopulo quondam Antonio quondam } \\ & & \text { Tomà }\end{array}$

$1062+60$ ser Francesco Rizzardoprlo quondam Antonio quondam papà

$6.3+59 \quad$ ser Vicenzo Calanarcopulo

$73+49$ ser Todorin Cuppa

$75+47$ ser Alessandro Chelmi capitano

$1567+55 \quad$ ser Antonio Lachanà Rossolimo

$67+55$ ser Colela Valsamachi

$70+52$ ser Zuane Rossolimo quondam misser Marco

$38 \quad 84$ ser Giacomo Conduri

$31 \quad 73$ ser Anzolo Valsamachi

$2964+58$ ser Lazaro Chelmi

3587 ser Dimitri Panà

$84+\quad 38$ ser Dimo Cuppa

2.5 $\quad 9.5$ ser Dario Panà

19103 ser Giorgo Panà

$2563+.37$ ser Nicolò Rizzardopulo quondam Andrea

$69+53$ ser Nicolò Panà

$65+5 y$ ser Francesco Panà

$96+26$ ser Zuane Rizzardopulo quondam Andrea ${ }^{3}$

29 9:3 ser Jani Panà

30 Furno publicati ad alta voce per me Marco Crasso Cancelliero li soprascritti segnati con croce come restati per li quindici di Spartea et Pessada com: qualli ch' hanno scosso maggior numsro di balle.

Davit Bembo Proveditor

Christophoro Crassà Sindico

35 Galeacio Peccator Sindico

Stamati Cymera Scindico della Comuniti.

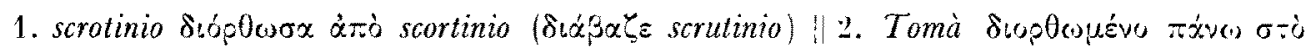

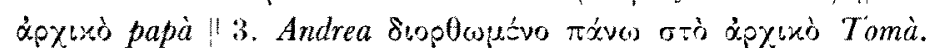




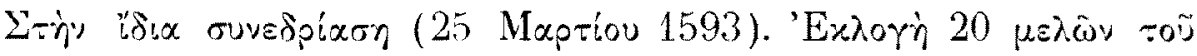

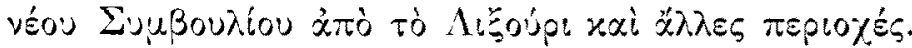

Q.10 Nell' istesso Conseglio fu estrato un'altro delli bolottini che sono rimasti et tocè in sort":

\section{LIXURI ET ALTRI LLOGI LONTANI}

Fatto il scrotinio ${ }^{1}$ per il Conseglio sono stati dalli consiglieri eletti el no5 minati li qui sotto annotati per esser balotatti nel numero; quali sono stati balotatti:

$\sigma \tau . \alpha^{\prime} D E$ SI $D E$ NO

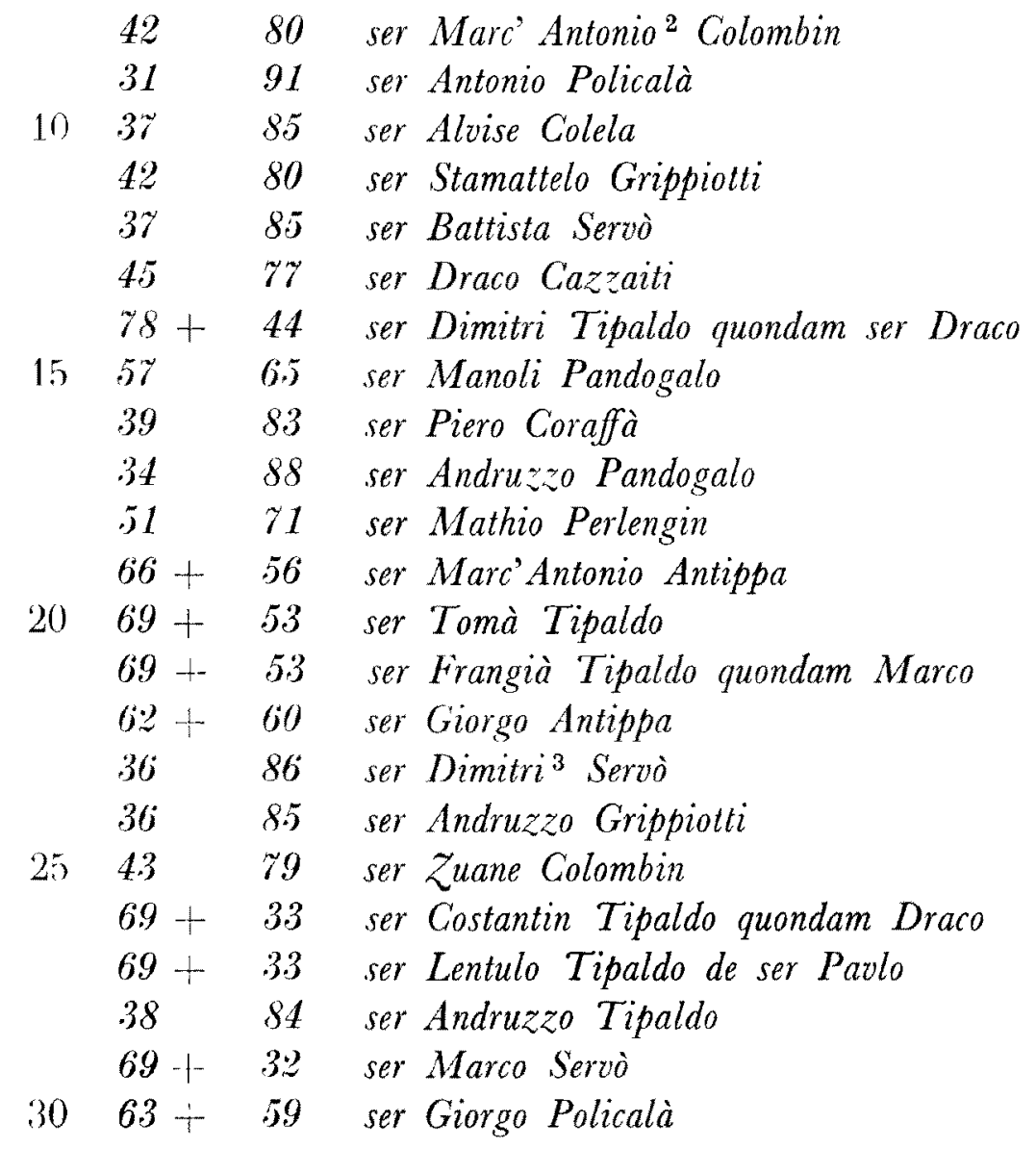

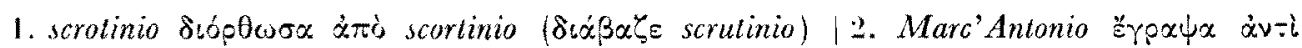

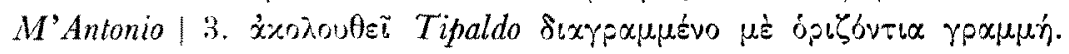


IIPAKTIKA EYMBOY $\sigma \tau \cdot \beta^{\prime} D E S I I N E$ NO

\begin{tabular}{|c|c|c|c|}
\hline & $75+$ & 47 & ser Alessandro Tipaldo \\
\hline & $64+$ & 58 & ser Franco Servò \\
\hline & 35 & 87 & ser Priamo Policalà \\
\hline 5 & 50 & $\gamma 2$ & ser Marc'Antonio Lusi del capitano \\
\hline & $67+$ & 55 & ser Zorzi Tipaldo quondam ser Draco \\
\hline & $83+$ & 39 & ser Alessandro Peccator \\
\hline & 48 & 74 & ser Frangià Lusi \\
\hline & 51 & $\pi 1$ & ser Timotheo Pandogalo \\
\hline 10 & 44 & 78 & ser Dimitri Rossolimo \\
\hline & 60 & 62 & ser Dimitri Tipaldo \\
\hline & $66+$ & 54 & ser Janicachi Tipaldo \\
\hline & $69+$ & 53 & ser Dimitri Lusi capitano \\
\hline & $62+$ & 60 & ser Teoffilo Servò \\
\hline 15 & 22 & 95 & ser Comino Rossolimo \\
\hline & $67+$ & 55 & ser Draco Tipaldo de ser Pavlo \\
\hline & 45 & 77 & ser Andrea Perlengin \\
\hline & 5.3 & 69 & ser Costantin Tipaldo quondam Antonio \\
\hline & 2.3 & 99 & ser Zuane Grippiotti \\
\hline 20 & $68+$ & 54 & ser Steffano Antippa \\
\hline & 45 & 77 & ser Battista Policalà \\
\hline & $\gamma 2+$ & 50 & ser Pavlo Tipaldo \\
\hline & $74+$ & 18 & ser Piero Tipaldo \\
\hline & 60 & 62 & ser Jorio Tipaldo \\
\hline
\end{tabular}

25 Furno publicati ad alta voce per me Cancelliero Crasso li sopranominati et segnati con croce come restati per li vinti di Lixuri et altri luoghi lontani havendo scosso maggior numero di balle.

Davit Bembo Proveditor

Christophoro Crassà Sindicho

30 Galeacio Peccator Sindico

Stamati Cymera Scindico della Comunità 


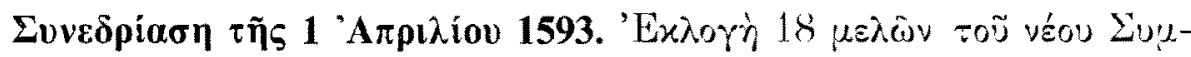

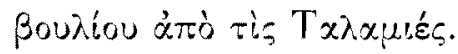

Congregato il Conseglio di questa Magnifica Communilà al numero di cento et settanta sie alla presentia del Clarissimo signor Davit Bembo honorato Proveditore premmsso il suon della campanna ordinaria per se-

5 guitar l'elettione delibrata nella parle de di 25 marzo 1593, nel qual Conseglio fu estrato in sorte uno delli cinque bolettini restati et toccò:

\section{TALAMIÉS}

Fatto scrotinio ${ }^{1}$ per il Consiglio sono stati dalli consiglieri eletti et nominati li qui sotto anotalti per esser balotatti nel numero; quali sono 10) stati balotatti:

$\sigma \approx \alpha^{\prime} D E$ SI $D E N O$

\begin{tabular}{|c|c|c|c|}
\hline & $171+$ & 6 & ser Zaccaria de Montesanto capitano \\
\hline & $162+$ & 14 & ser Nicolò de Montesanto \\
\hline \multirow{3}{*}{15} & $160+$ & 16 & ser Zuane de Montesanlo quondam Nicolò \\
\hline & 7.5 & 94 & ser Nicolò Marchetto \\
\hline & 90 & 86 & ser Alessandro Marcopulo quondam fanuzzo \\
\hline \multirow{7}{*}{20} & $164+$ & $1 \%$ & ser Zuane de Montesanto do Nicolò \\
\hline & 81 & 95 & ser Vardaramo de Montesanto \\
\hline & $1.36+$ & 40 & ser Andruzzo de Montesanto de papà \\
\hline & $136+$ & .38 & ser Zuane de Montesanto quondam Stamatti \\
\hline & 60 & 112 & ser Andrea Marchetto \\
\hline & $1+0+$ & 36 & ser Jani de Montesanto de papà \\
\hline & 94 & 82 & ser Marc'Antonio de Montesanto \\
\hline \multirow{3}{*}{25} & 50 & 125 & ser Zuane Marchetto \\
\hline & $126 \quad 1$ & 50 & ser Antonio de Montesanto quondam Vardaramo \\
\hline & $126-+-$ & 48 & ser Francesco de Montesanto \\
\hline
\end{tabular}

$142+28$ ser Antonello de Montesanto quondam Vicenzo

$144+.30$ ser Antonio de Montesanto quondam Apostoli

$\begin{array}{lll}30 & 81 \quad 94 \quad \text { ser Andrea de Montesanto }\end{array}$

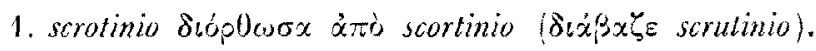




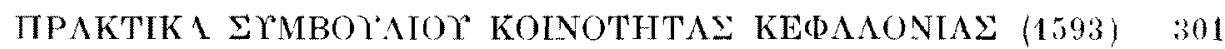

\begin{tabular}{|c|c|c|c|}
\hline \multicolumn{4}{|r|}{ in $d e$} \\
\hline & 34 & 142 & ser Fillippo Marchetto \\
\hline & 102 & 74 & ser Giorgo de Montesanto \\
\hline & $148+$ & 28 & ser Nicolò de Montesanto quondam Apostoli \\
\hline 5 & $134+$ & 32 & ser Ferrando de Montesanto \\
\hline & $146+$ & 39 & ser Michali Marcopulo ${ }^{1}$ \\
\hline & 31 & 145 & ser Giacomo Marchetto \\
\hline & 53 & 122 & ser Fani Marchetto \\
\hline & 60 & 113 & ser Petro de Montesanto \\
\hline 10 & $136+$ & 40 & ser Alessandro Marcopulo quondam Manoli \\
\hline & $140+$ & 34 & ser Antonio Marcopulo \\
\hline & 20 & 153 & ser Petro Marcopulo \\
\hline & $140-$ & 34 & de Montesanto de papà \\
\hline
\end{tabular}

Furno publicati ad alta voce per me Marco Crasso Cancelliero li soprascrit15 ti segnati con croce come restati per li disdotto di Talamiés come quelli ch' hanno scosso maggior numero di balle.

Davit Bembo Proveditor

Christophoro Crassà Sindico

Galeacio Peccator Sindico

20 Stamati Cymera Scindico della Communità

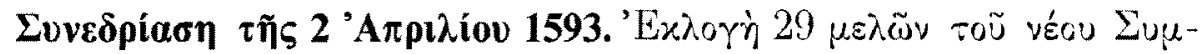

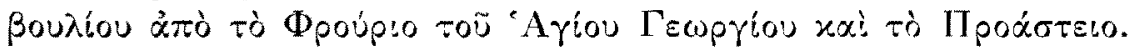

$0.11^{\mathrm{r}}$

1593 adi 2 Aprile

Congregato il Conseglio di questa Magnifica Communità al numero di cento e ottanta uno alla presentia del Clarissimo signor Davil Bembo honorato Proveditore premesso il suon della campanna ordinaria per seguitar

25 l'elettione deliberata nella parte de di 25 Marzo 1593, nel qual Conseglio fu estrato in sorte uno delli quattro bollettini restati et toccò:

\section{CASTELLO ET BORGO}

Falto scrotinio ${ }^{2}$ per il Conseglio sono stati dalli conseglieri eletti et nominati li qui sotto anotatti per esser balotatti nel numero; quali sono 30 stati balotatti ${ }^{3}$ :

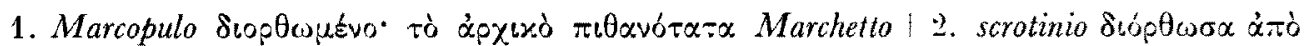

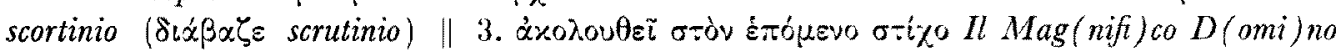

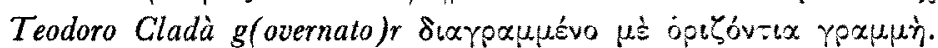




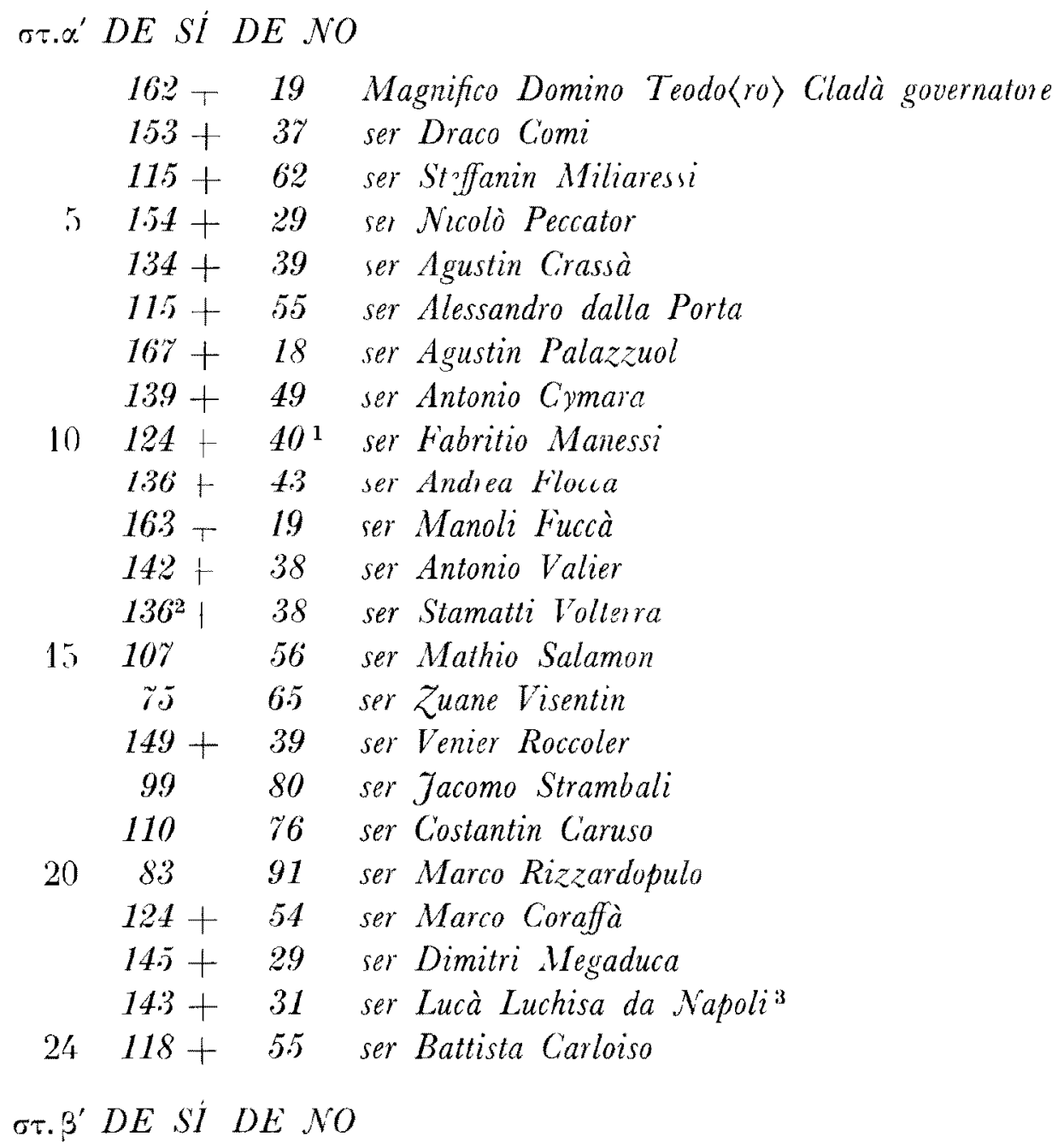

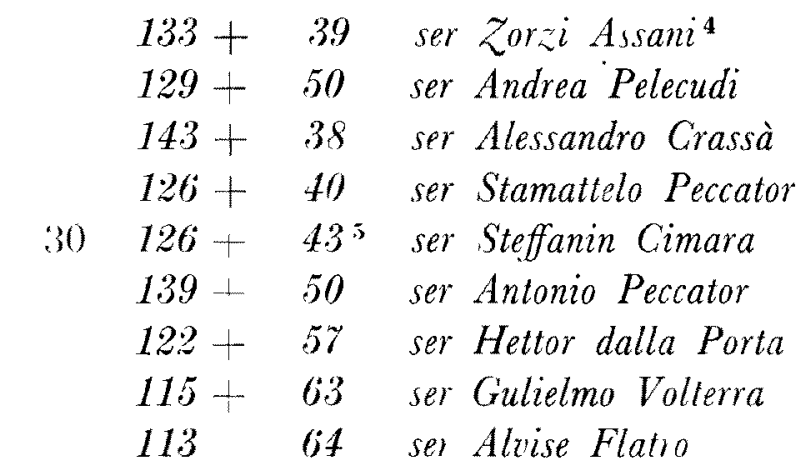

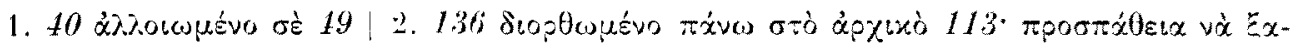

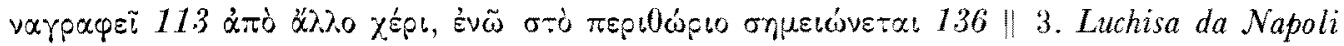

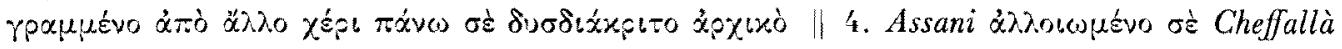

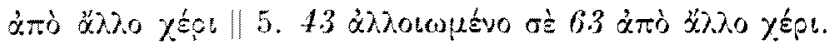


IIPAKTIKA ETMBOYION KOINOTITAZ KEФA NONIAE (1593) 303

$\begin{array}{lll}108 & 69 & \text { ser Piero Anino } \\ 156+ & 26 & \text { ser Zuane Crassà } \\ 115+ & 55 & \text { ser Zorzi Volterra } \\ 136+ & 41 & \text { ser Antonio Crassà }\end{array}$

$5 \quad$ Per l'esser l'hora tarda fu licentiato il Conseglio el stridatto per dimani.

Davil Bembo Proveditor

Christophoro Crassà Sindico

Galeacio Peccator Sindico

Stamati Cymera Scindico della Comunita

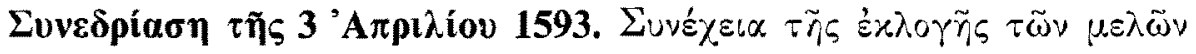

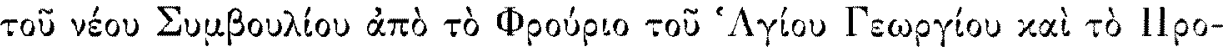

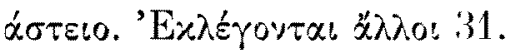

p. 11

1593 adi 3 aprile

Congregato il Conseglio di questa Magnifica Communità al numero di cento et settanta sei alla presentia del Clarissimo signor Proveditore premesso il suon della campanna ordinaria per seguitar la ballolatione principiata hieri di Castello et Borgo.

$$
\sigma \tau . \alpha^{\prime} D E S I ́ D E N O
$$

$138+38$ ser Damian Mingardo

$128+55$ ser Zorzi Cymara

$122+61$ ser Draco Crassà

$120+58$ ser Nadal Volterra

$20143+30$ ser Marc'Antonio Palazzuol

$118+5.3$ ser Nicolò Lion

$137+43$ ser Vangeli Crassà

$125+41$ ser Vicenzo Solomon

$123+55$ ser Francesco Volterra

$25142+41$ ser Andrea Fuccà

$134+34$ ser Marco Peccator

$116+60$ ser Francesco Gavran

$118+56$ ser Zuane dalla Porta

11368 ser Ventura Anino

$301.32+47$ ser Stamattello Crassà

$133+38$ ser Marcello Metaxà 


$$
\begin{array}{lll}
126+ & 51 & \text { ser Cecho Crassà } \\
126+ & 45 & \text { ser Lunardo Servò } \\
124+ & 52 & \text { ser Cristofforo Cymara } \\
150+ & 26 & \text { ser Steffanin Crassà }
\end{array}
$$

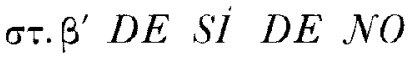

$$
\begin{aligned}
& 116+56 \text { ser Nicolò Volterra } \\
& 166+13 \text { ser Todorin Mingardo } \\
& 10183 \text { ser Zuane Caruso } \\
& 114+61 \text { ser Marco Manessi }{ }^{1} \\
& 10 \quad 113 \quad 53 \text { ser Galeazzo Miliaressi } \\
& 10265 \text { ser Hettor Coraffà } \\
& 122+32 \text { ser Francesco Dandolo } \\
& 1.54+26 \text { ser Dimitri Crassà } \\
& \text { 10.5 } 61 \text { ser Gerolimo Anino } \\
& 15129+46 \text { ser Mathio dalla Bionda } \\
& 175+4 \mathrm{ser}^{2} \\
& \text { - }-\operatorname{ser}^{3} \\
& 110 \quad 47 \text { ser Agustin Peccator } \\
& 143+27 \text { ser Fioravanti Crassà } \\
& \text { 20) } 89 \quad 56 \text { ser Paulin Capassà } \\
& \text { 121 + } 60 \text { ser Anzolo Mingardo } \\
& 118+60 \text { ser Todorin Cazzaiti } \\
& 125 \text { t- } 38 \text { ser Andrea Rafti } \\
& 125+34 \text { ser Draco Fuccà } \\
& 258590 \text { ser Francesco Salamon } \\
& 8588 \text { ser Gabriel Cazzari }
\end{aligned}
$$

Fu licentialo il Conseglio essendo l'hora tarda et stridatto per il giorno seguente.

Davit Bembo Proveditor

30 Christophoro Crassà Sindico

Galeacio Peccator Sindico

Stamati Cymera Scindico della Comunità.

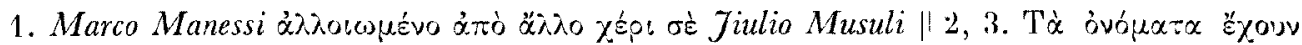

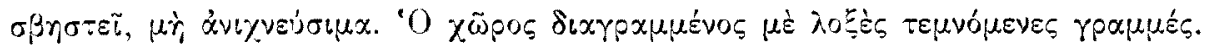




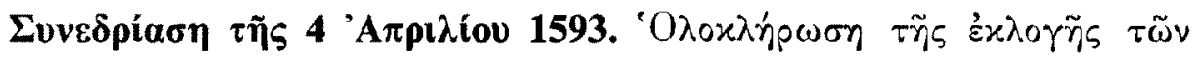

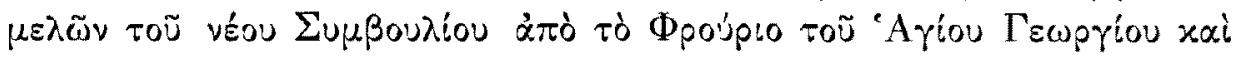

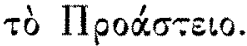

Congregato il Conseglio di questa Magnifica Communità al numero di cento quaranta otto ${ }^{1}$ alla presentia del Clarissimo signor Proveditore premesso il suon della campanna ordinaria per seguitar la ballotatione princi5 piata li giorni inanzi di Castello et Borgo.

$\sigma \tau . \alpha^{\prime} D E S I D E \mathcal{N O}$

\begin{tabular}{|c|c|c|c|}
\hline \multicolumn{2}{|c|}{70} & 78 & ser Domenico da Napoli \\
\hline \multirow{4}{*}{10} & 70 & 81 & ser Costantin Milonà \\
\hline & $118+$ & 30 & ser Zuane di Gratiosi \\
\hline & 97 & 64 & ser Lunardo Cambici \\
\hline & 50 & 93 & ser Nicolò Capiano \\
\hline \multirow{7}{*}{15} & 113 & 35 & ser Nicolò Cimera \\
\hline & 76 & 66 & ser Zuane Coraffà \\
\hline & 78 & 63 & ser Vico da Modon \\
\hline & 57 & 81 & ser Zan' Francesco Sarachinopulo \\
\hline & 93 & 38 & ser Andrea di Sardi \\
\hline & 85 & 49 & ser Bernardin Trech \\
\hline & 87 & 51 & ser Dardo Sdrigna \\
\hline \multirow{3}{*}{20} & 74 & 69 & ser Antonio Janulato \\
\hline & 88 & 51 & ser Nicolò Geracari \\
\hline & 31 & 109 & ser Nicolò Anino \\
\hline \multirow{8}{*}{25} & 94 & 48 & ser Zuane di Visentini rebalotado per error di balle \\
\hline & 30 & 108 & ser Francesco Sicilian \\
\hline & 68 & 71 & ser Battista Dimissiano \\
\hline & 80 & 57 & ser Federigo Vilaraut \\
\hline & 93 & 49 & ser Gerolimo Perlengin \\
\hline & 88 & 54 & ser Marco Crasso ${ }^{2}$ agionto $^{3}$ \\
\hline & 68 & 71 & ser Piero Carloiso \\
\hline & 54 & 88 & ser Anzolo Caruso \\
\hline
\end{tabular}

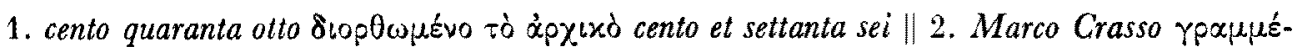

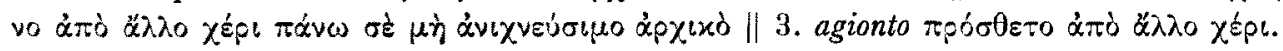




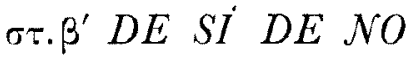

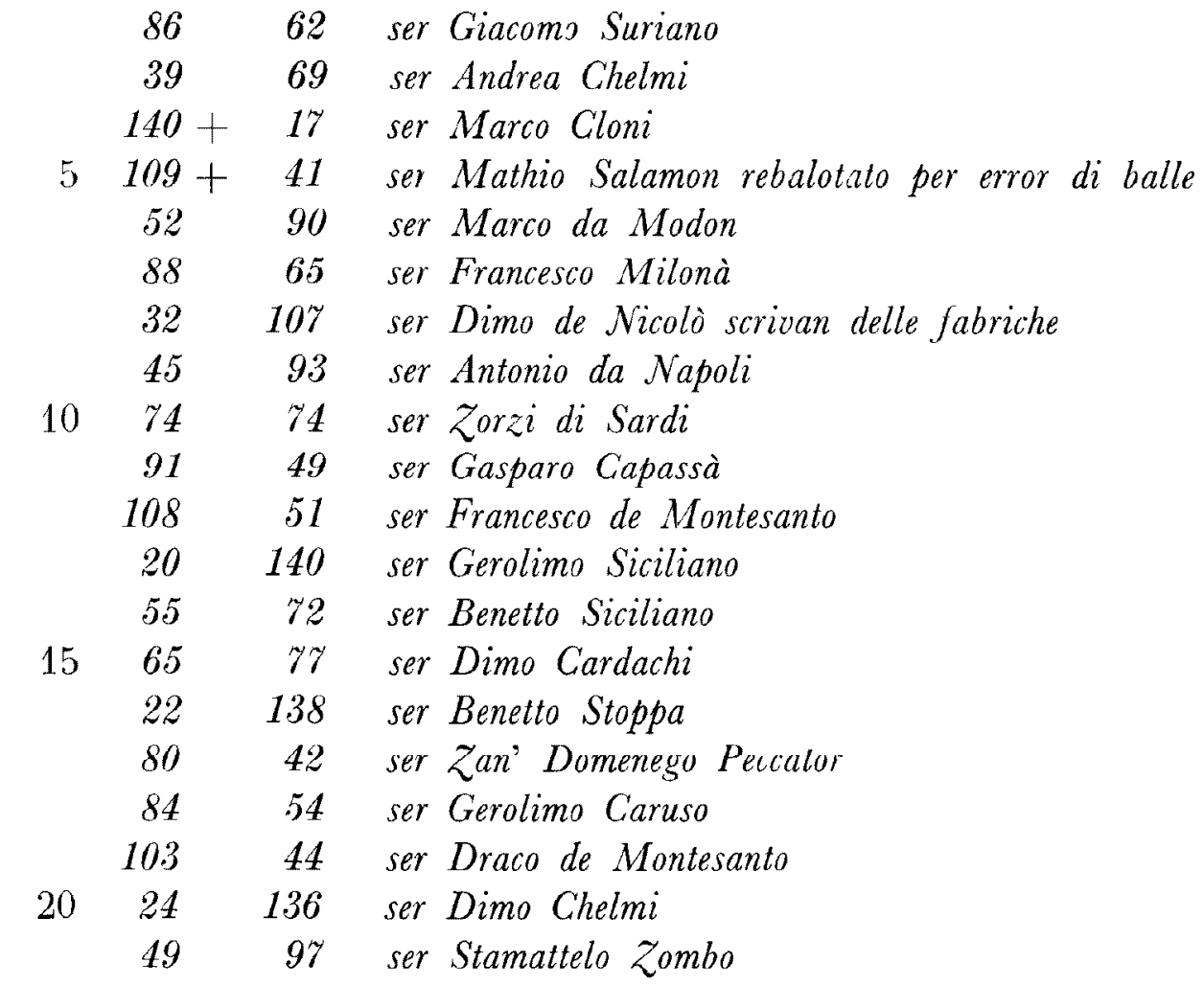

E perché fra ser Galeazzo Miliaressi, ser Nicolò Cymara, ser Alvise Flatro et ser Ventura Anino v'è successo parità di balle ciò̀ cento et tredici per uno, furno fatti quattro bollettini delli loro nomi, imbossolati et estrati 25 per sorte, venero et furno balotati ut infra:

$\begin{array}{rll}108 & 40 & \text { ser Ventura Anino } \\ 75 & 76 & \text { ser Alvise Flatro } \\ 103 & 57 & \text { ser Galeazzo Miliaressi } \\ 112+ & 36 & \text { ser Nicolo Cymara }\end{array}$

30 Li quali tutti segnati con croce di 2,3, e 4 d'aprile di Castello et Borgo al numero di sessandui furno ad alta voce per me Marco Crasso stridati per rimasi come quelli ch' hanno scosso magior numero di balle ${ }^{1}$.

Davit Bembo Proveditor

Christophoro Crassà Sindico

35 Galeacio Peccator Sindico

Stamati Cymera Scindico della Comunità

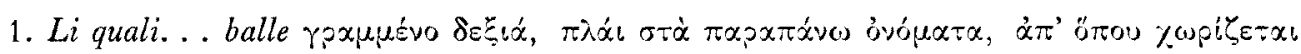

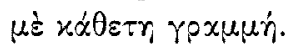




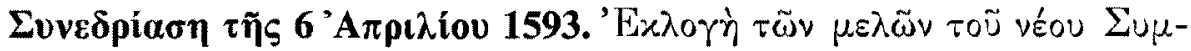

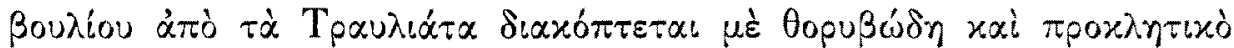

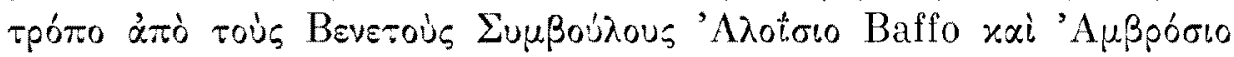
Corner. 'A

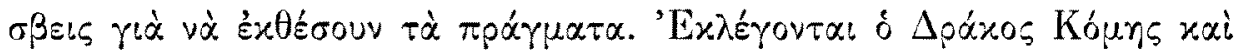
ó Nixó̀xoos II

$0.12^{v}$

Copia tratta da un foglio

datto a ms Cancelliero dal Censor

sottoscritto dal Clarissimo Proveditore et

Spettabili Sinici

5

Adi 6 aprile 1593

Congregato il Conseglio di questa Magnifica Communità al numero di cento e sesanta nove alla presentia del Clarissimo signor Proveditore premesso il suon della campana ordinaria per seguitar la balotattione et elettione conforme alla parte 25 marzo'93, fu estrato un bolettino di quelli che re-

10 storno et toccò in sorte come qui sotto:

DE SI DE NO

\section{TRAVLATA}

Per l'accidente tumulto grandissimo et strepito fatto per li Clarissimi signori Consiglieri Alvise Baffo et Ambroso Cornaro al presente in questo 20 Conseglio di Cefalonia, essendo venuti in tempo 〈che $\rangle$ era riddutto in grande numero per deliberar quel tanto per parte era stato in esso preso conforme alla deliberation dell' Eccellentissimo Senato de di $\langle 19 \text { gennaio } 1505\rangle^{1}$ et quanto è stato concesso a questa Spettabile Communità come nel capitolo octavo et proveder il buon stato d'esso, mentre si balotava hanno batuto alle

25 porte et con rumor entrati dentro, benché havessero recusato alla richiesta fatlali più volte d'intervenire, introdutti che furno, hanno con ignominiose parole di dishonore ${ }^{2}$ invilito il Conseglio, Sinici et tutti li cittadini, si come proposto havevano et ${ }^{3}$ insieme offeso uno d'essi cittadini con pugni nella

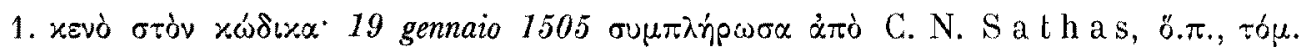

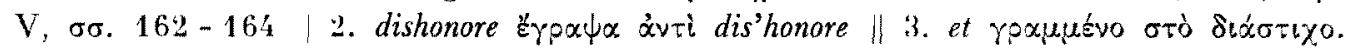


faccia, con il procurar confussione in esso usando diversi prottesti contra il Clarissimo Proveditore, il tutto mentre s'attrovasse al solito suo tribunale, il che richiedendo provisione per li Spettabili Sinici è stato proposto ut infra;

L'anderà parte che per questo Conseglio sia preso et statuito che si de-

5 vengi ad elettione di duoi ambasciatori per ricorer et comparer alli piedi di Sua Serenità per questo spetial effetto a ${ }^{1}$ rapresentar il pericolo importantissimo che poteva nascer et tutte quell'altre cose che sarano neccessarie si come particolarmente nella supplicatione sarano anotate et tectenus appre$.1 .3^{\mathrm{r}}$ sentate, devendossi partire $\|$ con prima occasione di passaggio, devendossi

10 impegnar l'istessi offitii della Communità per la spesa et pagamento loro.

Balotalta la soprascrilta parte, aperti li bossoli et numerate le balle in presentia di tutto il Conseglio sono state trovate a pro balle $n^{\circ}$ ottanta nove et contra quaranta, et fu presa.

Fatto immediate scrotinio ${ }^{2}$ sono stati proposti per ambasciatori l'infrascritti:

бг. $\alpha^{\prime} \quad$ ser Zorzi Volterra

ser Gradenigo dalla Porta

ser Venier Roccoler

ser Nicolò Peccator

$\sigma \tau . \beta^{\prime} \quad$ ser Cristofforo Crassà il qual non fu balotatto stante l'iscusa

20 ser Draco Comi

ser Antonio Valier

ser Alessandro dalla Porta

Et balotatti furno nel modo qui sotto annotato venuti per sorte:

$\sigma \tau . \alpha^{\prime} D E S I D E \mathcal{N O}$

$25 \quad 72 \quad 56$ ser Gradenigo dalla Porta

13109 ser Venier Roccoler

6464 ser Anlonio Valier

$108+18$ ser Nicolò Peccator

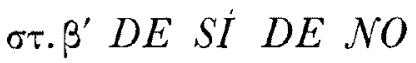

$\begin{array}{llll}30 & 51 & 7 \% & \text { ser Alessandro dalla Porta }\end{array}$

$48 \quad 74$ ser Zorzi Volterra

$115+10$ ser Draco Comi

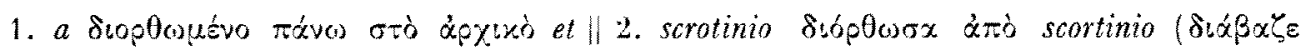
scrutinio). 
Rimasero per ambasciatori, com: quelli che scossero maggior numero di balle, ser DRACO COMI et ser NICOLO' PECATOR, a quali fu datto giuramento in debita forma di ricercar quel tanto è stato preso per la parte et ogni beneffitio della Communità senza rispetto d'alcuna sorte et qualità 5 di persona.

Davit Bembo Proveditor

Christophoro Crassà Sindico

Galeacio Peccator Sindico

Stamati Cymera Scindico della Comunità

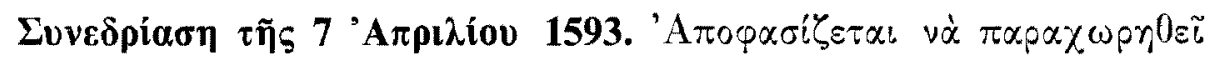

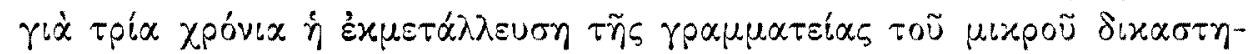

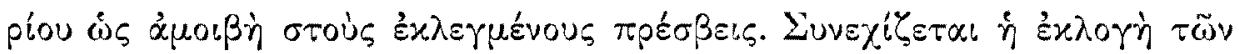

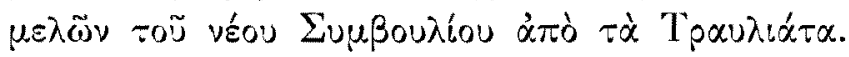

$\varphi .13^{\mathrm{*}}$

\section{3 adi 7 aprile}

Congregato il Conseglio di questa Magnifica Communità al numero di cento e otto alla presentia del Clarissimo signor Davit Bembo honorato Proveditore por continuar all'elettione et balotattione di quelli da Travlata, toccatoli in sorte et datto principio come nell'avanti Conseglio, nel quale

15 avant' ogn' altro per li spettabili Sinici è stato proposto ut infra:

Che conforme alla parte presa il giorno d'heri in questo Conseglio per pagamento delli signori Ambasciatori li sia assignato et deputato ${ }^{1}$ la Cancellaria della Corte Minor libera, senza tansa, per anni tre continui principiando però doppo che fornito haverano gl'attuali cancellieri; la qual Can-

20 cellaria possano far per nomo loro et far far ad altri come meglio li parerà non potendo ${ }^{2}$ però essercitarla altri che di quelli $\langle$ che $\rangle$ serano rimasti del Conseglio.

Balotata fu la soprascritta parte et tolse a pro balle cento et sei et contra due et fu presa.

25

Nomi di quelli ch'intervenero nel Conseglio

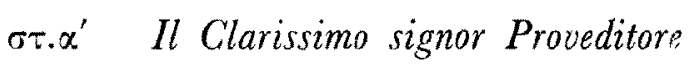

Il Spettabile signor Cristofforo Crassà

Il Spettabile signor Galeazzo Peccator

Il Spettabile signor Stamatti Cymara

Sinici

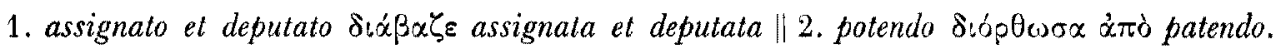




\author{
ser Zuane Visentin coadiutore \\ ser Stamatti Volterra \\ ser Agustin Crassà \\ ser Zorzi Volterra \\ 5 ser Andrea Capassà \\ $X$ ser Marc'Antonio Antippa \\ ser Zaccaria de Montesanto \\ ser Antonio Cambici \\ ser Nicolò Cambici \\ 10 ser Michel Rizzardopulo \\ ser Dimo Valiano \\ ser Giorgo Antippa \\ ser Lunardo Travlò \\ ser Stamatti Travlò de Federico \\ 15 ser Apostoli Travlò \\ $X X$ ser Francesco Rizzardopulo \\ $\sigma \tau . \beta^{\prime} \quad$ ser Francesco de Montesanto \\ ser Achile Rizzardopulo \\ ser Giorgo Combotecra \\ 20 ser Mathio Marcopulo \\ ser Agustin Marcopulo \\ ser Nicolò Travlò de Leo \\ ser Elia Maltese \\ ser Cristofforo Valiano \\ 25 ser Stamatti Metaxà \\ $X X X$ ser Stamattelo Carandinò \\ ser Alessandro Rizzardopulo \\ ser Tomaso Travlò \\ ser Petro Schiadà \\ 30 ser Jani Travlò \\ ser Giacomo Travlò quondam Andrea \\ ser Steffanin Cambici \\ ser Anastassi Michalitiano \\ ser Cristofforo Carandinò \\ 35 ser Gerolimo Carandinò \\ XXXX ser Nicolò de Montesanto \\ $\varphi .14^{\mathrm{r}}$ ser Marco Travlò \\ ser Draco Fuccà \\ ser Steffanin Miliaressi \\ 40 ser Gerolimo de Montesanto
}




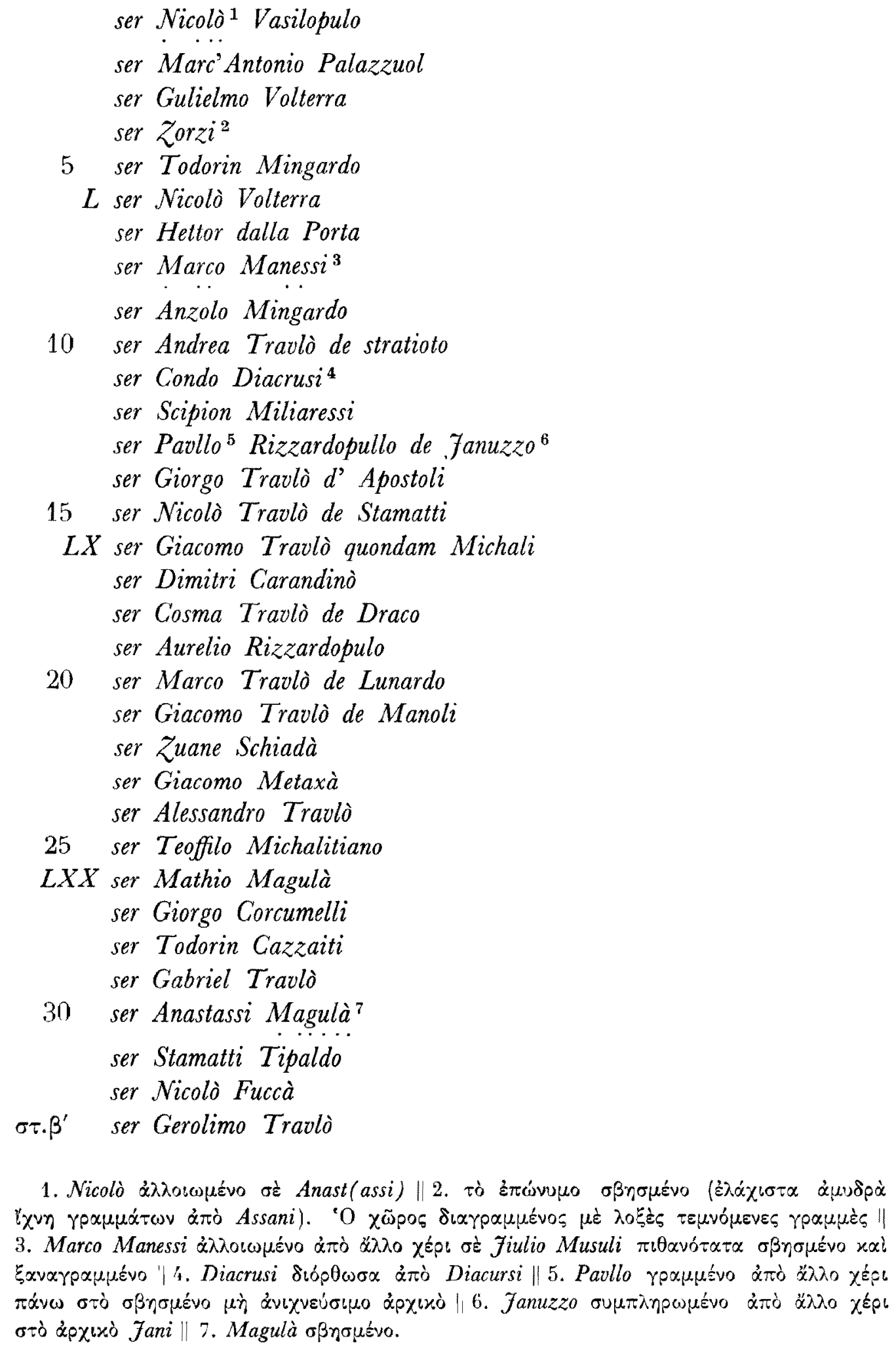




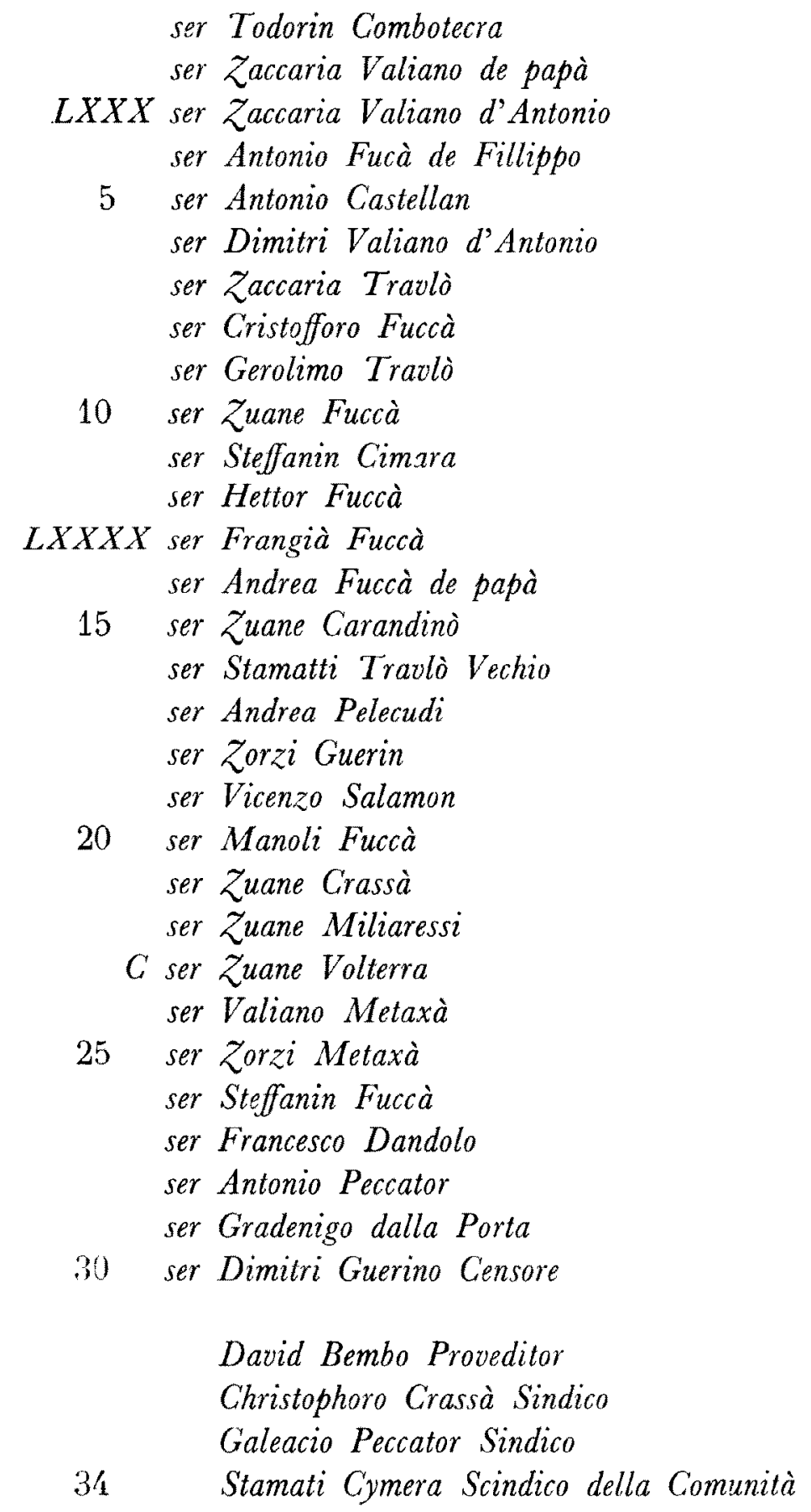

५.14 ${ }^{\mathrm{v}}$ Nell'istesso Conseglio fu principiato da novo alla balotattione interrotta il giorno sei aprile da quanto successe che $f u$ di: 


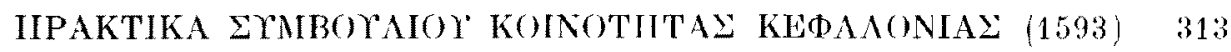

\section{TRAVLATA}

\begin{tabular}{|c|c|c|c|}
\hline \multirow{6}{*}{5} & $85+$ & 22 & ser Stamatti Travlò de Federico rebalotato \\
\hline & 50 & 58 & ser Gorolims Travlò de Manoli \\
\hline & $82+$ & 26 & ser Zuane Travlò de ${ }^{1}$ Tomaso \\
\hline & $94+$ & 15 & ser Stamatti Travlò Vechio \\
\hline & 54 & .56 & ser Leo Travlò \\
\hline & $93+$ & $1 . \tilde{\jmath}$ & ser Lunardo Travlò \\
\hline \multirow{3}{*}{10} & 41 & 67 & ser Zuan? Travlò d'Apsstoli \\
\hline & $87+$ & 21 & ser Giacoms Travlò de Manoli \\
\hline & 21 & 84 & ser Marco Travlò do Lucà \\
\hline \multirow{7}{*}{15} & 60 & 48 & ser Luccà Travlò \\
\hline & $87+$ & 18 & ser Tomaso Travlò \\
\hline & $79+$ & 27 & sor Zaccaria Travlò \\
\hline & $87+$ & 21 & ser Andruzzo Travlò de Stamalti \\
\hline & 7.3 & 34 & ser Marco Travlò de Zuane \\
\hline & $90+$ & 16 & ser Andruzzo Travlò de Nicolò \\
\hline & $99+$ & 9 & ser Giorgo Travlò quondam Apostoli \\
\hline
\end{tabular}

$\sigma \tau . \beta^{\prime} D E$ SI DE NO

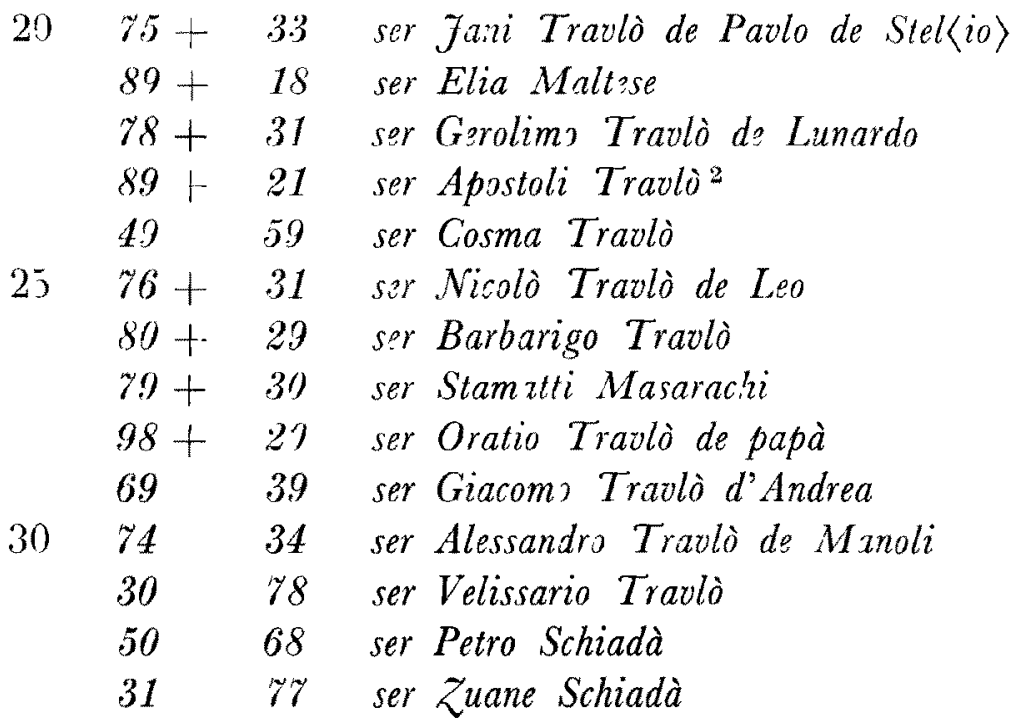

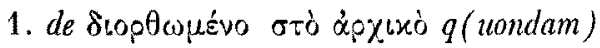

2. $\alpha \times 0 \lambda o v \theta \varepsilon i$ de Lunardo reb(alotato) $\cdot \delta\llcorner\alpha-$

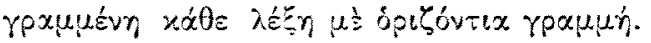


Furno li soprascritti segnati con croce con ser Zuane Masarachi capitano et ser Zorzi Travlò balotati alli 6 aprile publicati per rimassi al numero di vinti giusta la parte de dí 25 marzo '93.

Davit Bembo Proveditor

5 Christophoro Crassà Sindicho

Galeacio Peccator Sindico

Stamati Cymera Scindico della Comunità

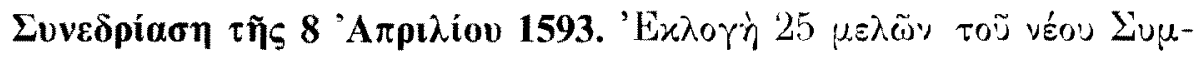

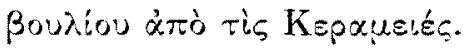

Congregato Conseglio di questa Magnifica Communità al numero di 10 cento e disdotto ${ }^{1}$ alla presentia del Clarissimo signor Davit Bembo honorato Provaditore per seguitar l'elettione el balottatione giusta la parte di 25 marzo 1593, fu cavato per sorte un bolettino delli restati et toccò:

\section{CHIERAMIÉS}

Onde scrotiniato ${ }^{2}$ il Conseglio furno eletti li sottoscritti et balotati ut 15 infra:

\begin{tabular}{|c|c|c|c|}
\hline \multicolumn{4}{|c|}{ 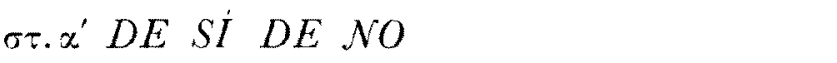 } \\
\hline & $113+$ & 5 & ser Valiano Metaxà \\
\hline & $112+$ & 6 & ser Giacomo Metaxà \\
\hline & $118+$ & 1 & ser Antonio Cambici \\
\hline 20 & $115+$ & .3 & ser Nicolò Cambici \\
\hline & $109+$ & 9 & ser Cristofforo Valiano \\
\hline & $109+$ & 9 & ser Nicolò Valiano \\
\hline & $99+$ & 19 & ser Mathio Marcopulo \\
\hline & $112+$ & $y$ & ser Michali Rizzardopulo \\
\hline 25 & $114+$ & 4 & ser Andrea Capassà \\
\hline & $112+$ & 6 & ser Stamatti Metaxà \\
\hline & $110+$ & 8 & ser Francesco Metaxà \\
\hline & 25 & 8.3 & ser Marco Valiano \\
\hline & $109+$ & $y$ & ser Achile Rizzardopulo \\
\hline
\end{tabular}

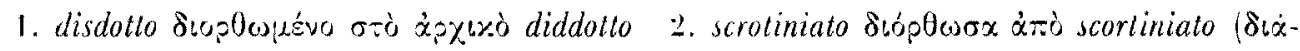

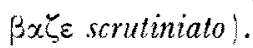




$\begin{array}{llll} & 84 & 34 & \text { ser Alvise Cambici } \\ & 93+ & 23 & \text { ser Stamatti Valiano } \\ 89^{1}+ & 27 & \text { ser Andruzzo Valiano } \\ 67 & 51 & \text { ser Steffano Cambici } \\ 5 \quad & 88+ & 30 & \text { ser Nicolò Marcopullo } \\ 89- & 29 & \text { ser Simo Cambici } \\ 93+ & 25 & \text { ser Nadal Rizzardopulo } \\ +25^{2} & 83 & \text { ser Giorgilà Dracopulo }\end{array}$

$\sigma \tau . \beta^{\prime} D E$ SI DE NO

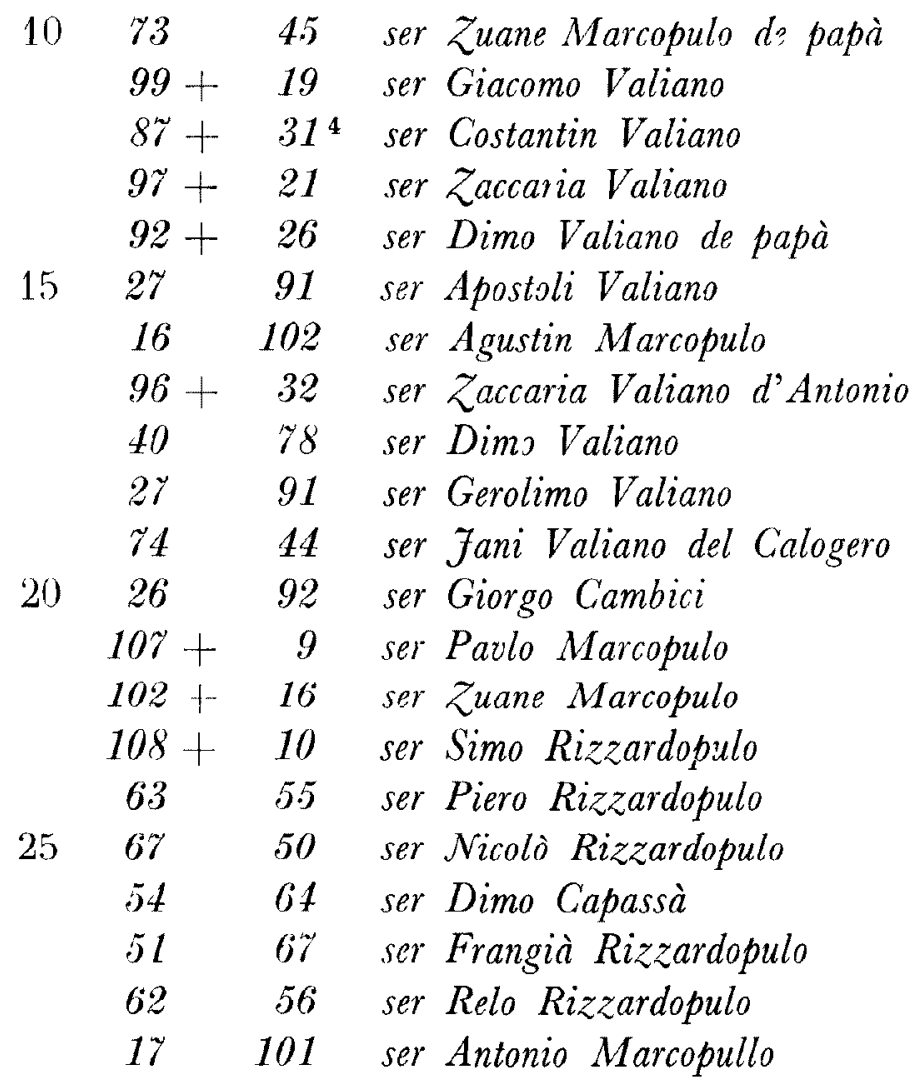

$30 \quad$ Furno publicati per m: Cancelliero ad alta voce li soprasritti segnati con croce come li vinti cinque restati in Chieramiés.

Davit Bembo Proveditor

Christophoro Crassà Sindico

Galeacio Peccator Sindico

35 Stamati Cymera Scindico della Comunità

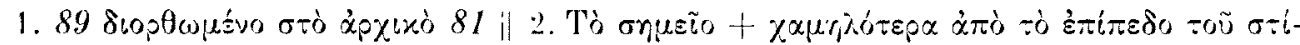

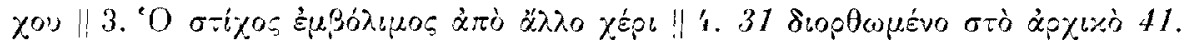




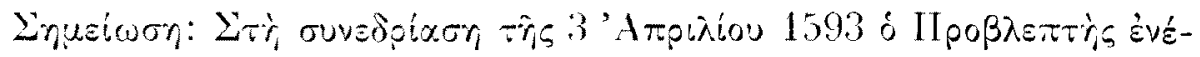

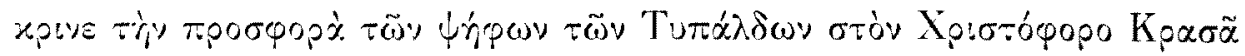

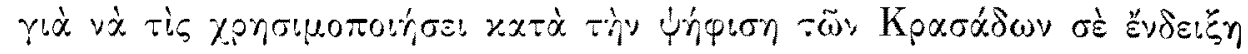

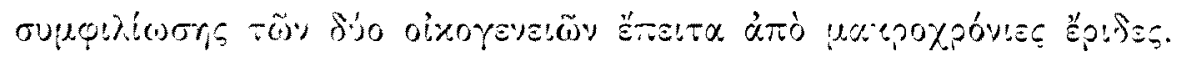

$\varphi .15^{\mathrm{v}} \quad$ Nota chs alli 3 aprile 1593, che furno balotatti quelli del Castello, comparse alla presentin del Clarissims signor Proveditore il signor Nicolo Peccator insiemo con misser Zorzi, Costantin, Alessandro, Costantin quondam ser Antonio, Andruzzo, Lontulo, Dracs, Stunatti, Zorzi, Piero el

5 Janachi Tipaldi et richiesero a Sua Signoria Clarissima ch' essendo stato per molt' anni questione importantissima fra la famiglia de magnifici Crassani et signori ${ }^{1}$ Tipaldi et seguito dall' una parte et dall' altra diverse risse, questione, ferrison et homicidii et havendo in essecution di parte dell' Eccelso Conseglio di X fatto pace et deventossi ballotlar ${ }^{2}$ li Crassani eletti,

10) acciò restino sicuri essi magnifici Crassani dell'amorevol proceder di loro Tipaldi, sincerità dell'anims loro et appaggatti compiutamente d'essi, sia per sua Signoria Clarissima concesso che le balle d'essi Tipaldi siano por il spettabile signor Cristofforo Crassà posti nel si nelle ${ }^{3}$ balotattioni ${ }^{4}$ sole d'essi magnifici Crassani.

15 Il che inteso per Sua Signoria Clarissima et essendo benissimo informato di quanto veramente hanno esposto parendoli dimanda giusta et che sii per portar bon affetto et amorevolezza tra esse famiglie et quiete conforme alla deliberata volontà di Sua Serenità in generale et in particolare dell'Eccelso Conseglio di $X$ che con tanta efficaria ha sopra ciò scritto, ha concesso che

20 sempre cho sarano balotatti li magnifici signori Crassani per questa volta tanto possino li Tipaldi dar le loro balle ad esso magnifico signor Crist?fforo acciò per essi balotati.

Illico fu per li signori Tipaldi ad un' ad uno alla presentia d'esso Clarissimo signor Proveditore datto ${ }^{5}$ le balle ad esso magnifico signor Cristofforo,

$25\langle$ il〉 quale con esse ha balotatto a nom. loro ogni fata che furno balotatti essi magnifici Crassani.

Davit Bembo Proveditor

Christophoro Crassa Sindico

Galeacio Peccator Sindico

3) Stamati Cimera Scindico della Comunità

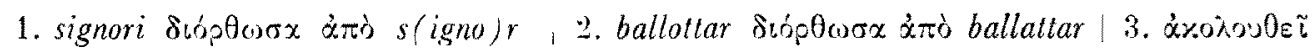

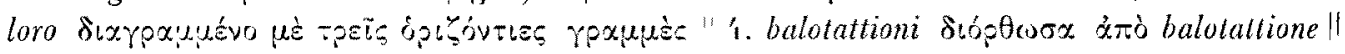
5. fu. . datio $\delta \iota \alpha \beta \alpha \varsigma$ furono datte. 


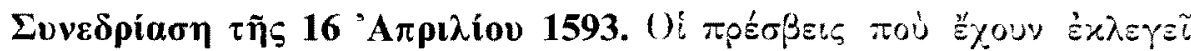

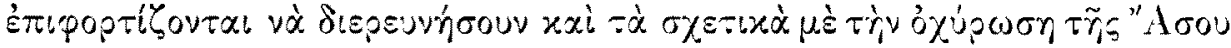

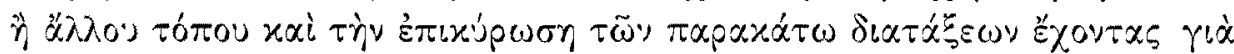

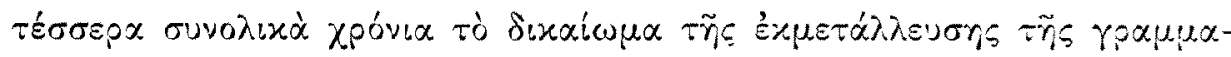

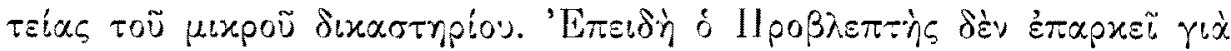

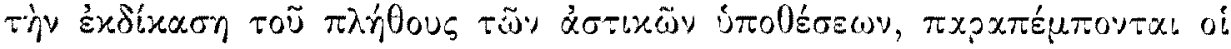

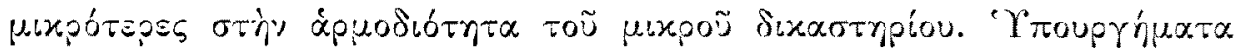

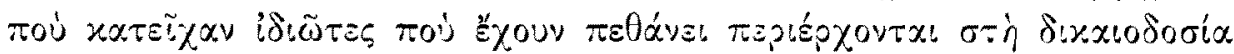

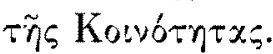

Q. $16^{r}$

Adi 16 aprile 1.593

Congregato Conseglio di questa Magnifica Communità alla presentia del Clarissimo signor Davit Bem[bo] honorato Proveditore al numero di cento et vinti sette per deliberar quanto qui sotto et continuar alle balotationi ${ }^{1}$ 5 giusta la parte 2.5 marzo ${ }^{2} 1593$, dove dall $\left.i\right\rceil$ spettabili signori Sinici di detta Magnifica Communità unanimi tutti tre fu rappresentato et letto ${ }^{3}$ l'infrascritti capitoli et balotatti ut infra:

Che conforme alla parte presa li 6 instante, che s' habbia a mandar ambasciatori a piedi di Sua Serenità il signor Draco Comi et il signor Nicolò

10 Peccator Visconte, eletti da questo Conseglio per la causa contenuta nella parte, li sia appresso detto carico di poter ricercar et dimandar l'ultima rissolutione et deliberatione in proposito della fortiffication d'Asso o altro locco giusta la forma della supplicatione mandata al signor Zuane Cymara et oltre ciò ricercar in nome di questa Magnifica Communità la confermatione

15 dell'infrascritti capitoli; alli quali ambasciatori sia datto per salario et pagamento un'altr'anno con gl'altri tre giusta la parte pres $[a]$ sotto 7 instante, siché in tutto sia per anni quattro la Cancellaria Inferiore libe [ra] potendo affittarla per li predett'anni quattro o disponer d'essa come li parerà, con espressa dichiaratione che non definiendo l'espeditione do tutte le predette

20 cose et non portando risposta cadano in pena essi signori Ambasciatori di restituir tutto il dinaro ch'havessero estratto o tolto da detta Cancellaria Inferiore et esser privi per anni dieci d'ogni offitio et beneffitio di questo Conseglio non potend'essi o alcuno di loro esser più del numero d'esso.

\section{Omessa la supplicatione}

25 Che essendo cresciute le differentie civili di questo populo in tal maniera che il Clarissimo Reggimento a pena può espedire delle cento una con molto

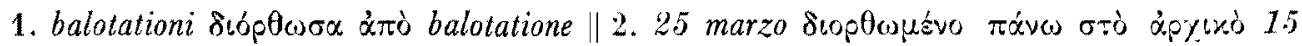

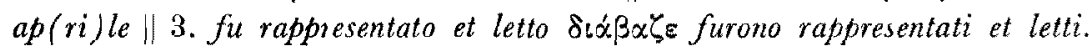


danno delli poveri litteganti $i$ quali si strussiano continuamente in litle spsndendo ogni loro sostan|tia」 in pagamento d'advocati et altro et poi al fine non hanno il più dille vo[lte] l'espeditione, sia ${ }^{\mathbf{1}}$ concesso che li giudici della Corte Inferiore quali son[o] cittadini eletti da questo Conseglio in $\varphi .16^{\vee}$ essecution della parte dell'Eccellentissimo Senato $\|$ possino ove giudicano ${ }^{2}$ sin ducati tre farls sin a ducati sei conforme a quanto s'osserva et $\grave{c}$ stato concesso al Zante. Et questo doppo che sarà adempito la gratia del Crasso di modo che non habbia più intoresse nella Cancellaria.

Che doppo la morte di donna Stams Xomeritena ${ }^{3}$ moglie de ser Dimo

10 de Nicolò l'offitio del scrivano delle fabriche resti a questa Communità, acciò possino elegger annualmente uno del corpo del ${ }^{4}$ Conseglio ad administrar esso carico con tutta l'uttilità, salarii et emolumenti suoi; restando d'ogni intatto ch'Iddio non voglia la Communità obligata o mala administratione.

Che doppo la morte delli Guerini l'offitio dell'Interprete ${ }^{5}$ della lingua

15 greca di questo locco resti a questa Communità sí come è stato concesso alla città del Zante.

Che doppo la morte delle signore ***:** l'offitio delli commandamenti greci resti a questa Communilà d'elegger uno cilladino ad anno per anno ad essercitarlo, accioché questi poveri cittadini si possano per queste vie su-

20 stentarsi et mantenersi nel modo che in diverse città di questo felicissimo Dominio s'osserva et spetialmente alla città del Zante.

Che doppo la morte di misser Marcello Metaxà cittadino di questo locco, che per gratia particolare ha l'offitio del masser delli libri della Cancellaria, sia concesso esso offitio alla Comnunità, acciò d'anno in anno possi elegger 25 uno d'essi cittadini a tal carico.

Li quali capitoli letti ad intslligentia d'ogn' uno et balotalti hebbero a pro balle $n^{0}$ cento et vinti tie et contra quattro et fu presa.

Davit Bembo Proveditor

Christophoro Crassà Sindico

30 Galeacio Peccator Sindico

Stamati Cymera Scindico della Comunità

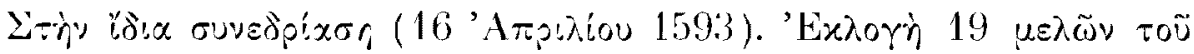
véou $\Sigma$ u.

1. $\alpha$ ×

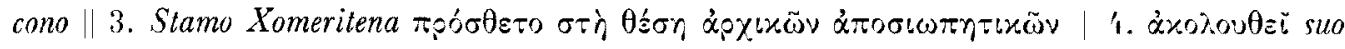

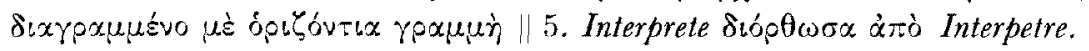


Adí detts

Nell'istesso Consiglio fu estrato l'ultimo bolettino restato delle compartita et toccò:

\section{LACHITRES, METAXATA}

5

et altri luoghi

Onde fatto in esso Conseglio scrotinio ${ }^{1}$ furno elett li qui sotto et balotati in sorte videlicet:

$$
\begin{aligned}
& \sigma \tau . \varepsilon^{\prime} D E \text { SI } D E \mathcal{N O} \\
& 103+24 \text { ser Nicolò Barbati governature } \\
& 10 \quad \% 8 \quad 54 \text { ser Dimitri Vinari capilano } \\
& 107+19 \text { ser Marco Fuccà capitano } \\
& 102+24 \text { ser Zuane Carandinò } \\
& 98+28 \text { ser Stamatti Combotecra }{ }^{2} \text { capitano } \\
& 60 \quad 66 \text { ser }\left[\text { An]astassi Magulà }{ }^{3}\right.
\end{aligned}
$$

20

$113+13$ ser Andrea Fuccà de papà

$116+10$ ser Steffanin Fuccà del capitano

$102+24$ ser Pavlo Barbati capitano

$74 \quad 40$ ser Andrea Combotecra capitano

$25 \quad 88 \quad 38$

$53 \quad 73$ ser Petro Metaxà

$86^{5} \quad 39$ ser Manoli Metaxà

$90+\quad 35$ ser Marc'Antonio Corcumelli

$56 \quad 70$ ser Apostoli Vinari capitano

30 «ser Jullio Musuli $\rrbracket^{6}$

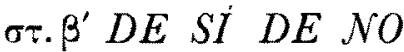

$\begin{array}{lll}90+ & 3 j & \text { ser Metaxà Metaxà } \\ 85 & 40 & \text { ser Nicolò Vergotin } \\ 83 & 42 & \text { ser Scipion Miliaressi }\end{array}$

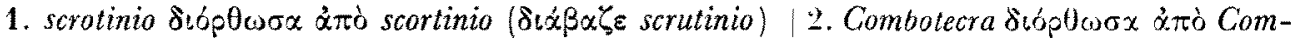

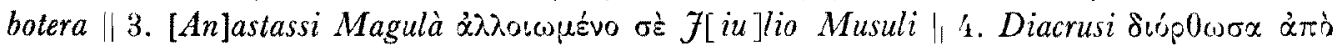

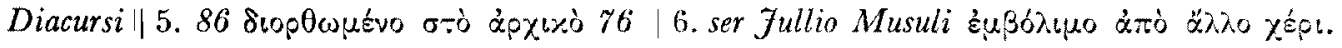




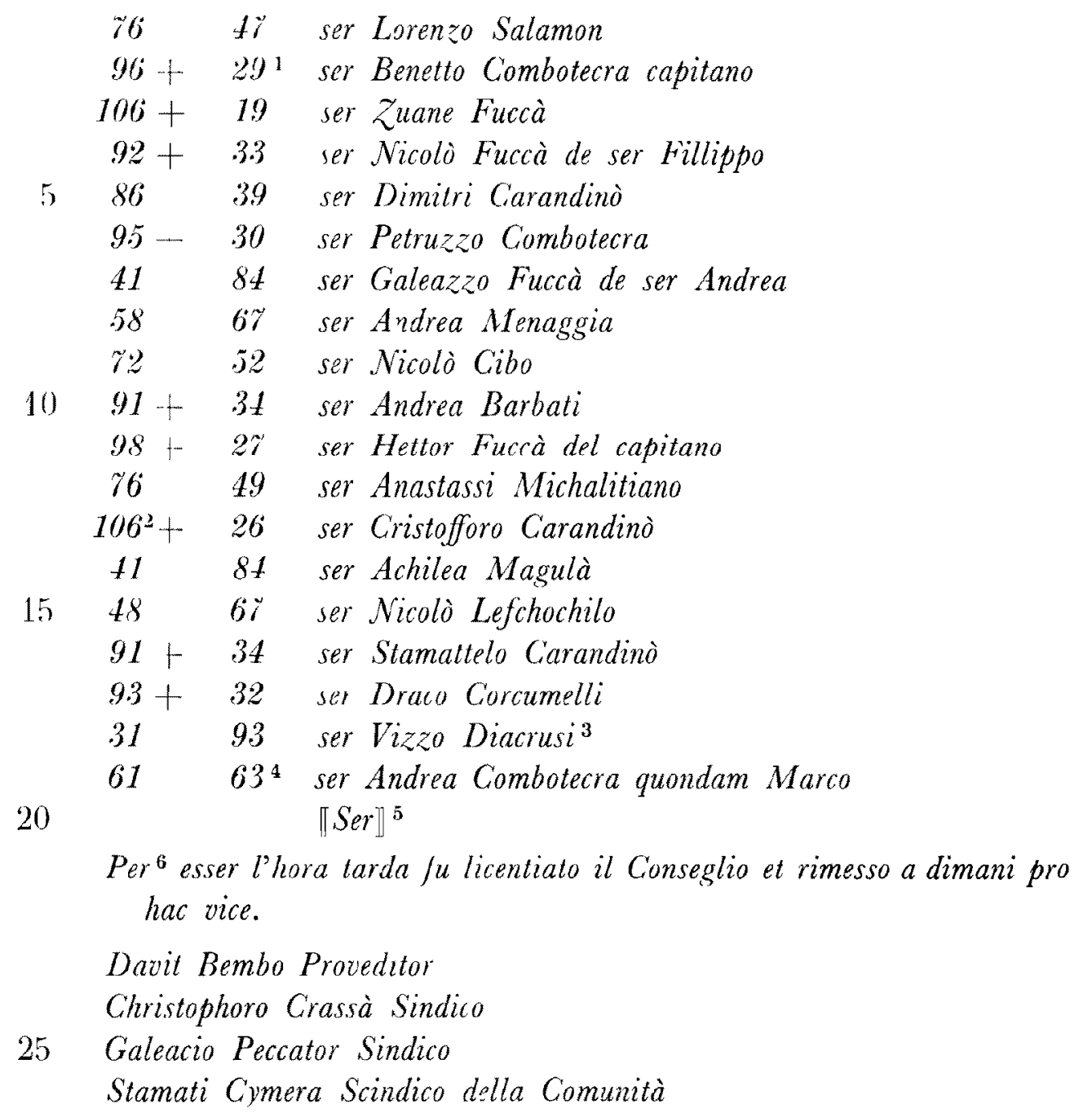

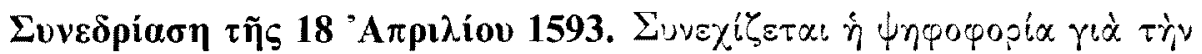

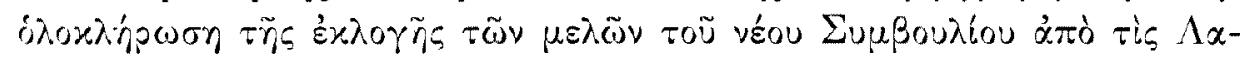

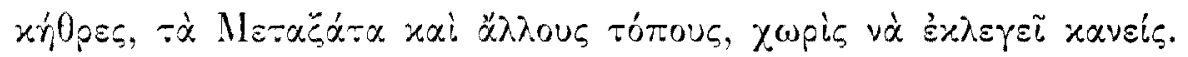

Congregato il Conseglio di quesla Magnifica Communità alla presentia del Clarissimo signor Proveditor al numero di 97 per seguitar alla balota30) tione di Lachitres, Metaxata et altri luoghi ${ }^{1}$ fu principiato ut infra seguitando il principiato, videlicet:

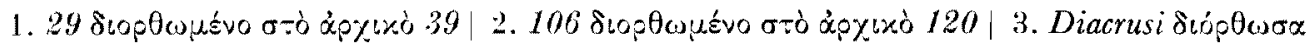

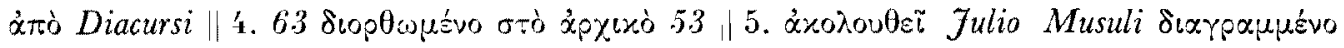

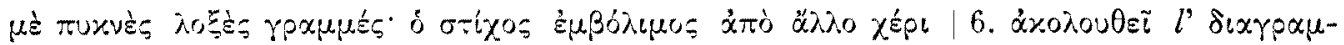

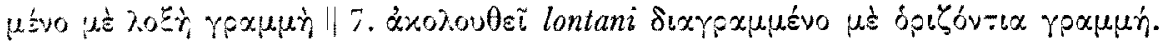


$\sigma \tau . \alpha^{\prime} D E S I$ DE NO

$\begin{array}{cccc} & 19 & 78 & \text { ser Anastassi Diacrusi } \\ & 60 & 40 & \text { ser Zorzi Metaxà Vecchio } \\ 5 & 33 & 67 & \text { ser Zorzi Vinari de capitano } \\ & 30 & 70 & \text { ser Nicolò Maguld } \\ & 55 & 45 & \text { ser Gasparo Corcumeli } \\ & 34 & 66 & \text { ser Giorgo Spata } \\ & 48 & 52 & \text { ser Cesare Vinari } \\ 10 & 26 & 74 & \text { ser Zorzi Salamon } \\ & 34 & 66 & \text { ser Nicolò Fuccà de Pasco } \\ & 48 & 52 & \text { ser Zorzi Menagia }\end{array}$

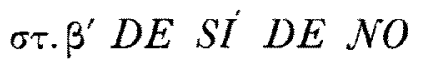

\begin{tabular}{rlll} 
& 17 & 83 & ser Polo Cibo \\
15 & 38 & 62 & ser Mathio Magulà \\
& 47 & 53 & ser Giorgo Combotecra \\
& 54 & 46 & ser Costantin Vergotin \\
& 30 & 70 & ser Fani Catavati \\
20 & 57 & 43 & ser Dimitri Lefcochilo \\
& 47 & 53 & ser Apostoli Magulà \\
& 40 & $60^{2}$ & ser Jani Cangadi de Sgurò \\
27 & $73^{3}$ & ser Manoli Soymiri \\
& 50 & 50 & ser Gerolimo Corcumelli \\
& \multicolumn{3}{l}{ Per esser l'hora tarda fu rimessa a dimani. }
\end{tabular}

25 Davit Bembo Proveditor

Christophoro Crassà Sindico

Galeacio Peccator Sindico

Stamati Cymera Scindico della Comunità.

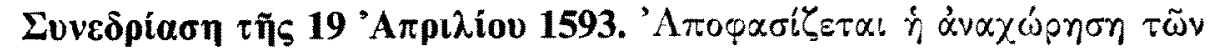

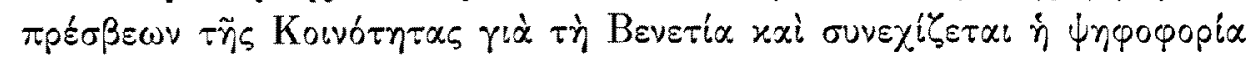

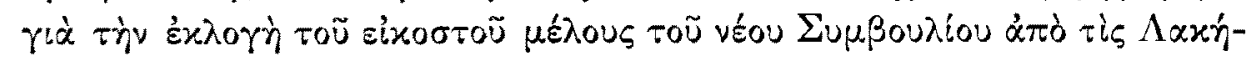

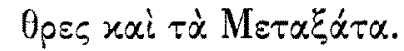

$\varphi .18^{\mathrm{r}}$

Adi 19 aprile 1593

30 Congregato Conseglio di questa Magnifica Communità alla presentia

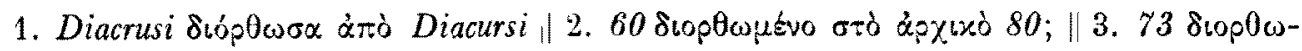

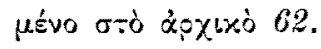


del Clarissimo signor Proveditore per deliberar quanto qui sotto et continuar alla balotattione lasciata hieri et furno in Conseglio cento et sedici.

Fu posta dalli spettabili Sinici tutti tre unanimi ' l'infrascritta parte:

Che per molti convenienti rispetti sia terminato et presa parte che $i$

5 signori Ambasciatori si debbano partir con prima sicura occasione rappresentandossi o alla più con il Clarissimo signor Proveditore potendo quando alcuno d'essi fusse impedito l'altro solo partirsi per le cause et effelti presi et deliberati in questo Conseglio come per le parti si vede et questo in caso ch' il Clarissimo signor Proveditor successore non desse conseglio in caso d'

10 impedimento ${ }^{2}$ d'alcuno d'essi signori Ambasciatori per poter venir a nova creatione in loco suo devendo essi ambascialori o ambasciator uniti et separati negotiar $\langle e t\rangle$ trattar tutte le cose a loro comesse sino alla espeditione non si potendo partir fino alla deffinitione di tutti li capitoli et ordini presi come di sopra et che sarano a loro consignati; et questo sotto pena di ducati cin-

15 quanta $^{3}$ applicati all'Arsenal et restituir tutto il tolto alla Communità.

La qual balotatta tolse a pro balle cento e cinque et contra undici; et fu presa.

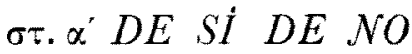

Fu continuato ${ }^{4}$ la balotatione ut infra:

$\begin{array}{cccc}20 & 53 & 59 & \text { ser Gabriel Fuccà del capitano } \\ & 18 & 94 & \text { ser Andruzzo Magulà } \\ & 37 & 75 & \text { ser Gerolimo Carandinò } \\ & 33 & 81 & \text { ser Zorzi Menaggia } \\ & 46 & 66 & \text { ser Todoro Combotecra } \\ 25 & 75 & 38 & \text { ser Anastassi Fuccà } \\ & 74 & 38 & \text { ser Cristofforo Fuccà }\end{array}$

$\sigma \tau . \beta^{\prime} D E S I D E N O$

$45 \quad 67$ ser Giorgo Fuccà

$54 \quad 58$ ser Antonio $^{5}$ Fuccà

$30 \quad 50 \quad 62$ ser Antonio Castellan

$37 \quad 29$ ser Gini Renessi

2591 ser Zuane ${ }^{6}$ Cangadi

$37 \quad 29$ ser Nicolo Corcumeli

Rebalotati furno

$35 \quad 63 \quad 52 \quad$ ser Todorin Castellan

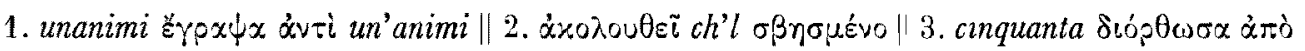

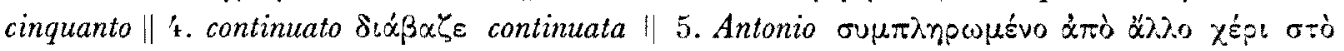

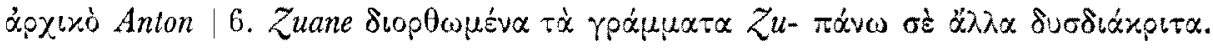




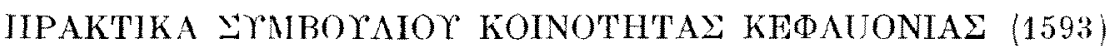

$84+32$ ser Marc'Antonio Mavrichi

Publicati furno ad alta voce per li $X X$ rimasi in Lachilres et Metaxata quelli ch'hanno segnato ${ }^{1}$ con croce $^{2}$.

Davit Bembo Proveditor

5 Christophoro Crassà Sindicho

Galeacio Peccator Sindico

Stamati Cymera Scindico della Comunità.

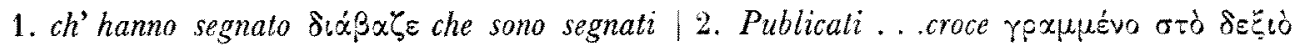

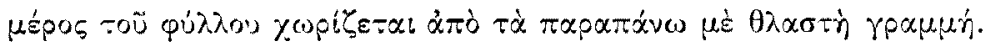




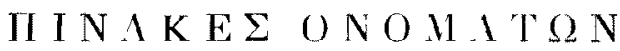

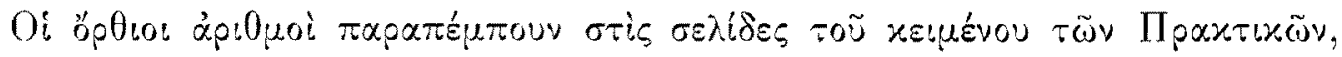

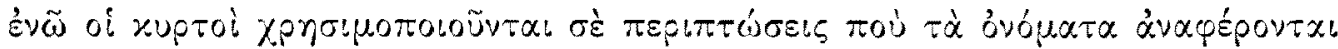

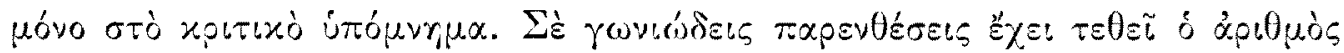

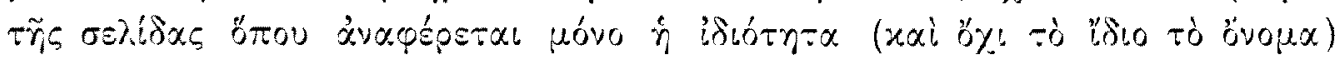

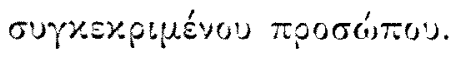

\section{II POY $\Omega \Pi \Omega \mathrm{N}$}

L Lramo Paplo 286.

Aggiandriti Luć̀ quondam ser Stamalli 278.

Anina Geralimo 296, 304.

Anino Gerolimo de ser Piero 279.

Anino Nicoló 305 .

Anino Nicolò de ser Piero 279.

Anino Piero 303.

Anino Piero quondam ser Andrea 279.

Anino Ventura 303, 306.

Anino Ventura de ser Piero 279.

Antippa Agustin de papà 289.

Antippa Fillippo 289.

Antippa Giorgo 298, 310.

Antippa Januzzo de papà 289.

Antippa Manoli quondam ser Dirno 289.

Antippa Mare'Antonio 298, 310.

Antippa Marc'Antonio de ser Giorgo 287.

Antippa Spiro quondam ser Michali 289.

Antippa Stamattelo quondam ser Antonello 289.

Antippa Steffano 299.

Antippa Todorin de papà 289.

Antippa Velissario quondam ser Antonello 289.

Antippa Zuane 289.

Asani Zorzi $\beta \lambda$. Assani.

Assani (Zorzi) 279, 302, 311.

Baffo Alvise, consigliere 307.

Barbati Andrea 320.

Barbati Andrea, capitano 280.

Barbati Nicolò, governatore 280, 319.
Barbati Paslo, capilano $280,319$.

Bembo Navit, proveditore 274, $\langle 275\rangle$, $276,291,\langle 293\rangle, 297,299,300,301$, $303,304,\langle 305\rangle, 306,\langle 307\rangle,\langle 308\rangle$, $309,312,314,315,316,317,318$, $320,321,\langle 322\rangle, 323$.

Bianco Batlista quondam ser Cosmo 279.

Bianco Piero 29 '.

Bianco Piero quondam ser Cosmo $\geq 79$.

Bianco Zam' Batlista 296.

Calanarcopulo Giacnmo 289

Calanarcopulo Vicenzo $289,297$.

Callò $\beta$ 入. Mistro Callo.

Cambici Alvise 315.

Cambiri Alvise de se" Antonio 278.

Cambici Antonio 310, 31't.

Cambici Antonio quondam ser Zuune 278.

Cambici Giorgo 315.

Cambici Giorgo de ser Alessandro 281.

Cambici Giorgo de ser Alvise 287.

Cambici Lazaro de ser Alessandro 281.

Cambici Lunardo 295, 305.

Cambici Nicolò 295, 310,311.

Cambici Nicolò de ser Antonio 278.

Cambici Simo(n) 295, 315.

Cambici Simon quondam ser Lunardo 287 .

Cambici Stamati quondam papà Giongo 281.

Cambici Steffanin 294, 310.

Cambici Steffanin quondam ser Lunardo 287.

Cambici Steffano 315. 
Cangadi Jani de (ser) Sgurò 282, 321 .

Cangadi Zane 289.

Cangadi Zuane 322.

Capassà Andrea 296, 310, 314.

Capassà Andrea quondam ser Michali 285.

Capassà Dimo 281, 315.

Capassà Gasparo 306.

Capassà Gosparo quondam ser Giorgilà 279.

Capassà Pavlin 304.

Capassì Paslin quondam ser Criorgilà 279.

Capiano Nicolo 305.

Carandinò Cristofforo 310,320 .

Carandino Cristofforo quondam ser spi. lioti 282

Carandinò Demutri $311,320$.

Carandinò Gorolimo 310,322 .

Carandinò Stamaltelo 310, 320 .

Carandinò Stamattelo de ser Zuane 282.

Carandinò Zuane 282, 312, 319.

Cardachi Dimo 288, 306

Carloiso Battista 302.

Carloiso Piero 305

Caruso Ansolo 287, 305.

Caruso Costantin 287, 302 .

Caruso Francesco 287.

Caruso Gerolimo 287, 306.

Caruso Marc'Antonio 287.

Caruso Nicolò 287.

Caruso Teochari 287.

Caruso Todorin 287.

Caruso Zuane 287, 30'

Castellan Antonio 312, 322.

Castellan Todorin $\beta \lambda$. Castellano.

Castellano Antonio de ser Nicolò 286.

Castellano Todorin 319, 322.

Castellano Todorin quondam ser Antonio 286

Catavati Jani 321.

Cazzaiti Draco 28's, 298.

Cazzaiti Marc'Antonio quondam ser $Z u$ ane 289.

Cazzaiti Todorin 284, 294, 304, 311.

Cazzaiti Zuane de papà 289

Cazzari Gabriel 278, 30؛.

Cazzari Manoli 29'.
Cazzari Nicoló quondam ser Jani 278.

Cheffalà (Zorzi) 279, 302.

Cheffald Zorzi d(e ser) .1ndrea 278, 294, 280 .

Chelmi Alessandro 280 .

Chelmi Alessandro, capitano 297.

Chelmi Andrea 306.

Chelme Andrea del capitano 280.

Chelmi Demetrio 293.

Chelmi Dimo 306.

Chelmi Lazaro 280, 297.

Cibo Nicolò 295, 320 .

Cibo Nucolò quondam ser Lazaro 282.

Cibo Polo 287, 321.

Cibo Teoffilo 287.

Cicilian Gerolimo 293.

Cicilian Zuane 296

Cimara Stamattello, sinico, $\beta \lambda$. Cymera Stamati.

Cimara Steffanin 302, 312, $\beta \lambda$. xai Cymara.

C'imera Nicolò $\beta \lambda$. Cymara.

Cladà Teodoro, governatore 277, 301,302.

Cloni Marco 279, 306.

Cochini Zurai 287

Coccoli Guorgo d(e ser) Antonio 283, 284. Coccoli Piero quondam ser Nicolò 286.

Cocolt Dimitri de ser Jani 28't.

Cocoli Jani 284.

Cocoli Jani quondam ser Nicolo 286.

Cocoll Nicolò quondam ser Stamatti 286. Cocoli Zuane quondam ser Nicolò 286. Coidà Marco 289.

Coidà Piero 289.

Coidà Zuane 289.

Colela Aloise 298.

Colombin Marc'Anlonio 283, 298.

Colombin Marc'Antonio quondam ser Nicolò 285.

Colombin Zanico quondam ser Nicolo 285. Colombin Zorzi quondam ser Nicolò 285.

Colombin Zuane 298.

Combotecra Andrea, capitano 280, 319. Combotecra Andrea quondam Marco 320.

Combotecra Andrea quondam ser Mar$\operatorname{tin} 282$.

Combolecra Benello, capilano 280, 294, 320. 
Combotecra Draco del capitano 282.

Comboterra Ciorgo 310, 321.

Combotecra Giorgo quondam ser Nirolò 282.

Combotecra llettor del capttano 28:.

Combotecra Petruzzo 320.

Combotecra Pelruzzo, capitano 280.

Combotecra Stamatti, captano $280,319$.

Combotecra Todorm 312.

Cambotecra Todoro 32.2.

Combotecra Todoro quondam ser Marlin 282.

Comi Draco 277, 302, 308, ambasctatore $309,317,\langle 322\rangle$.

Condur Giacomo 297.

Coraffà Heltor 296, 30'.

Coraffà IIettor quondam ser Beno 279.

Coraffà Marco 302.

Coraffà Marco quondam ser Beno 279.

Coraffà Massimınıano quondam ser Todorin 286.

Coraffà Plero 298.

Coraffà Zanetto quondam ser Beno 279.

Coraffà Zuane 305.

Corcumeli Gasnaro 321.

Corcumeli Gasparo del protopapa 289.

Corcumell Gerolımo del protopapa 289.

Corcumell Glorgo de ser Dimo 289.

Corcumele Marc'Antonio del protopapa 289.

Corrumelı Nicolò 322.

Corcumell Zuane del protopapa 289.

Corcurnellı Antonio de ser Giorgo 289.

Corcumelle Draco 320.

Corcumell L Draco de ser Giorgo 289

Corcumell Francesco de ser Glorgo 289.

Corcumell Gerolimo 296, 321.

Corcumell Giorgo 311, 319.

Corcumelle Marr'Antanio 319.

Cornaro Ambroso, consigliere 307.

Crassà Agustin 302, 310.

Crassà Agustın fu de ser Antonto 277.

Crassà Alessandro 296, 302.

Crassà Alessandro fu de ser Cuacomo 277.

Crassà Antonio 303.

Crassà Antonio fu de ser Julvo 277

Crassà Cecho 304.
Crassà Cristofforo (Crustoforo, Christophoro), sindeco $\langle 274\rangle, 275,276,291$, $293,297,299,301,303,304,306$, $\langle 307\rangle 308,309,312,314,315$, $316,\langle 317\rangle, 318,320,321,\langle 322\rangle$, $3 \geq 3$

Crassà Dario, proveditor alla sanità 276.

Crassc̀ Dumutri 30'.

Crassà Dimutr fu de ser Lucrà 277.

Crassà Draco 303.

Crassà Draco de ser Dario 277.

Crassà Fiorasantz $30^{\prime}$.

Crassù Fioravanti de ser Agustin 277.

Crassà Francesco de ser Darlo 277.

Crassì Francesco fu de ser Florlo 277.

Crassà Soffianò, canceluere 276.

Crassà Stamattello 303.

Crassà Stamattello de ser Agustın 277.

Crassà Steffanin 30 '.

Crassì Steffanin fu de ser Francesco 277.

Crassà Tulio, giustızıere 276 .

Crassì Vangeli 303.

Crassì Vangeli fu de ser Francesco 273. Crassà Korzl $\beta \lambda$. Da Crassà.

Crassì Zuane 293, 303, 312.

Crassà Zuane fu de ser Francesco 277.

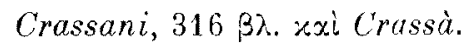

Crasso (Marco) 305, 318.

Crasso Marco, cancellero 275, 276, 293, $297,299,301,306,\langle 307\rangle,\langle 315\rangle$.

Crseopergi Jant 290.

Cuppa Dımo 283, 297.

Cuppa Draco 283.

Cuppa Todorın 283, 297.

Cymara Antonro 291, 302.

Cymara Antonıo quondam ser Marco 278 .

Cymara Cristofforo 293, 301.

Cymara Cristofforo quondam ser Ma. then 278.

Cymara Nicolò 305, 306.

Cymara Nicolò quondam ser Marro 278.

Cymara Stamate, sindlco, $3 \lambda$. Cymera.

Cymara stamattelo, sindico, $\beta \lambda$. Cymera stumati.

Cymara Steffanin de ser Stamallelo 278, 


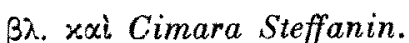

Cymara Zorzi 296, 303.

Cymara Zorzi de ser Stamattelo 278.

Cymara Zuan(n)e, ambasatore 276, 317.

Cymera Stamati, sindico $\langle 274\rangle, 275$, $276,291,293,297,299,301,303$, $304,306,\langle 307\rangle\langle 308\rangle, 309,312$, $313,315,316\langle 317\rangle, 318,320,321$, $\langle 322\rangle, 323$.

Da Crassà Zorzi 289.

Dalla Bionda Mathio $\beta \lambda$. Della Bionda.

Dalla Porta Alessandro 302, 308.

Dalla Porta Alessandro quondam ser Agustin 278.

Dalla Porta Gradenigo 294, 308, 312.

Dalla Porta Gradenigo, proveditor alla sanità 276.

Dalla Porta Hettor 302, 311.

Dalla Porta Hettor, capitano, quondam ser Covazzo 278.

Dalla Porta Zuane 303.

Dalla Porta Zuane quondam ser Agustin 278.

Da Modon Marco 283, 306.

Da Modon Velissario 283.

Da Modon Vico 283, 305.

Da Napoli Antonio 294, 296, 306.

Da Napoli Antonio, bombardier 283.

Da Napoli Domenego, sargente delle cernide 279.

Da Napoli Domenico 296, 305.

Da Napoli Stamatti, sartor 283.

Dandolo Francesco 296, 304, 312.

Dandolo Francesco quondam ser Orlando 279.

Dandolo Orlando quondam ser Andrea 279.

Da Paris Dimitri 294.

Da Paris Dimitri, bombardier et spader 288.

Della Bionda Mathio quondam ser Antonello 287, 304 .

De Montesanto Andrea 300.

De Montesanto Andrea quondam ser $\mathrm{Ni}$ coló 284.

De Monlesanto Andruzzo de(l) papà 283,300 .
De Montesanto Antonello quondam $V i$ cenzo 300.

De Montesanto Antonelo 286.

De Montesanto Antonio quondam (ser) Apostoli 285, 300.

De Montesanto Antonio quondam Vardaramo 300.

De Monlesanto Battista quondam ser Marco 286.

De Montesanto Draco 306.

De Montesanto Draco de papà Leo 279.

De Montesanto Ferrando 301.

De Montesanto Francesco $300,306,310$.

De Montesanto Francesco de papà Leo 279.

De Montesanto Gerolimo 310.

De Montesanto Gerolimo de papà 301.

De Montesanto Gerolimo del protopapa 285.

De Montesanto Giorgo 301.

De Montesanto Jani de papà 300 .

De Montesanto Jani de protopapa 285.

De Montesanto Marc'Antonio 283, 300.

De Montesanto Nicolò 295, 300, 310.

De Montesanto Nicolo quondam (ser) Apostoli 285, 301.

De Montesanto Petro 301.

De Montesanto Steffanin 301.

De Montesanto Steffanin quondam misser Stamatti 285.

De Montesanto Vardaramo 300.

De Montesanto Zaccaria 310.

De Montesanto Zaccaria, capitano 300.

De Montesanto Zaccaria quondam ser Vicenzo 285.

De Montesanto Zanetto 285.

De Montesanto Zuane de Nicolò 300.

De Montesanto Zuane quondam (ser) Nicold 285, 300.

De Montesanto Zuane quondam (ser) Stamati 286, 300.

De Nicolò Dimo, scrivan delle fabriche $279,306,318$.

Diacrusi Anastassi 321.

Diacrusi Condo 295, 311, 319.

Diacrusi Condo de ser Giorgo 284.

Diacrusi Giorgo de ser Condo 284. 
Diacrusi Vizzo 320.

Diacrusi Vizzo quondam ser Teoffilatto 284.

Di Gratiosi Zuane 305.

Di Gratiosi Zuane quondam ser Agustin 278.

Di (?) Lefteri Stathi 290.

Dimissiano Battista 305.

Dimissiano Rattista de ser Panagin 289.

Dimissiano Zuanne, roadiutore alli comandamenti 277.

Di Sardi Andrea 278, 293, 305.

Di Sardi Zorzi 306.

Di Visentini Marc'Antonio 294.

Di Visentini Zuane 296, 305.

Di Zentilini Marin 296.

Di Zentilini Marin, signore alle strade 277.

Dracopulo Giorgilà 315 .

Englesi Nicolò quondam ser Jani 288.

Fiscardi Bortolamio 279, 293.

Flatro Alvise 288, 302, 306.

Flocca Andrea 293, 302.

Flocca Andrea quondam ser Mathio 277.

Flocca Mathio, censor 274, 275, 276, 293.

Flocca Mathio de misser Andrea 274.

Fucà Anastassi del capitano Lunardo 282.

Fucà Antonio de (ser) Fillippo 284́, $295,312$.

Fucà Gabriel del capitano Lunardo 282,

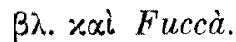

Fucà Hettor del capitano Lunardo 282,

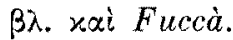

Fucà Steffanin del capitano Lunardo

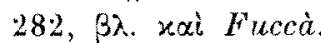

Fuccà Anastassi 322 .

Fuccà Andrea 303.

Fuccà Andrea quondam ser Galeazzo 280.

Fuccà Andrea de papà 312, 319.

Fuccà Andrea del protopapa 282.

Fuccà Antonio 322.

Fuccà Antonio de ser Fillippo $\beta \lambda$. Fucà.

Fuccà Antonio quondam ser Frangià 282.
Fuccà Cristodulo de ser Antonio 282 .

Fuccà Cristolforo 312,322 .

Fuccà Draco 304, 310.

Furcà Draco quondam ser Alessandro 286.

Fuccà Frangià 312, 319.

Fucrà Frangià de ser Antonio 282.

Fuccà Gabriel del capitano 322, $\beta \lambda$, xai Fucà.

Fuccà Galeazzo de ser Andrea 282, 320.

Fuccà Galeazzo del capilano Marco 282.

Fuccà Giorgo 295, 32:.

Fuccà Giorgo de ser Fillippo 28'

Fucè̀ Hettor 312.

Furcà Hettor del capttano $320, \beta \lambda . \gamma x i$ Fucà.

Fuccà Manoli 302, 312.

Fuccà Manoli fu del capitano 279.

Fucrà Marco, capitano 280, 319.

Fuccà Nirolò 311.

Fuccà Nicolò de ser Fillippo 28/, 294, 320.

Fuccà Nicolò de Pasro :3:1.

Fuccà Nicolò quondam ser Pasco 285.

Fuccà Orlando quondam ser Hettor 282.

Fuccà Steffanin 312.

Fuecà Steffanin d(e ser) Antonio $28: 2$, 319.

Fuccà Steffanm del capitano $319, \beta \lambda$. y.xi Fucà.

Fuccà Steffanin quondam ser Hettor 282. Fuccì Todossi de ser Antonio 282.

Fuccà Zuane 312, 320.

Furè̀ Zuane quondam ser Hettor 282.

Gasman Francesco 278, 29:3,303.

Gavrilopullo Nicolò 293.

Gavrilopullo Nirolo, giustiziere 276.

Geracari Nicolò 289, 305.

Grippiouti Andrea de ser Zuane $28 \%$.

Grippiotti Andruzzo 298.

Grippiotti Lazartn de ser Zuane 286

Grippiotti Stamattelo 298.

Grippiotti Stamaltelo de ser Zuane 281.

Grippiolli Zuane 299.

Grippiolli Zuane quondam ser Andrea 281.

Guerin Zorzi 296, 312. 
Guerin Zorzi, canceliere 276.

Guerini $318, \beta \lambda$. xai Guerin(o).

Guerino Demetrio (Dimitri), censor $274,275,276,291,293,\langle 307\rangle 312$.

Guerrino Demetrio, $\beta \lambda$. Guerino.

Sanulato Antonio $285,305$.

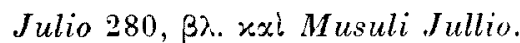

Lachanà Rossolimo Antonio $\beta \lambda$. Rossolımo Lachanà.

Lefchochilo Dimo quondam ser Piero 286.

Lefchochilo Nicolò 320 .

Lefchochilo Nicolò de ser Cosmeto 289.

Lefcochilo Dimitri 321.

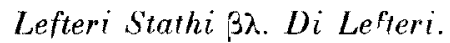

Lion Nicold 303.

Lion Nicoló quondam ser Marin 278.

Lirathinopulo Stamati 280, 289.

Lombardo Francesco, cittadin 281.

Luccà 283.

Luchisa Lucà da Napoli 302.

Lusi Dimitri, capitano 280, 299.

Lusi Frangià 299.

Lusi Frangià de ser Stamatti 280.

Lusi Marc'Antonio del capitano 280, 299.

Lusi Nicolò, capitano 280 .

Magulà Achulea 320.

Magulà Achilea quondam ser Franco 282 .

Magulà Anaslassi 288, 311, 319.

.Magulà Andruzzo 284, 322.

Magulà Apostoli 28', 321.

Magulà Marco 295.

Magulà Mathio 284, 311, 321.

Magulà Nicolò 321 .

Magulà Nicolò quondam ser Franco 288.

Maltese Elia 289, 310, 313.

Manessi Fabritio 279, 302.

Manessi Marco 279, 294, 304, 311.

Marchetto 301.

Marchetto Andrea 300.

Marchetto Fillippo 301

Marcetto Giacomo 301.

Marchelto Jani 301.

Marchetto Marco de ser Giacomo 287.
Marchetto Michali de ser Giaromo 285 .

Marchetto Nicalò 300.

Marchetto Nicolò de ser Giacomo 287.

Marchetto Zuane 300.

Marchello Zuane de papù ser Filippo 285.

Marcopullo Antonio 315.

Marcopullo Nicolò 295, 315.

Marcopullo Nicolò de papà 295.

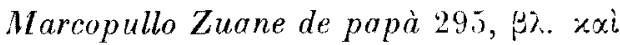
Marcopulo.

Marcopullo Zuane quondam ser Sileffa$\operatorname{nin} 287$.

Marcopulo Agustin 310, 315.

Marropulo Alessandro 295.

Marcopulo Alessandro quondam (ser) Januzzo 285, 300.

Marcopulo Alessandro de ser Manoli 285.

Marcopulo Alessandro quondam Manoli 301 .

Marcopulo Anionio 301.

Marcopulo Calogero quondam ser Steffanin 288.

Marcopulo Mathio 282, 310, 311.

Marcopulo Michali 301.

Mreropulo Michali quondam ser Giorgi 285 .

Marcopulo Pasto 315.

Marcopulo Paslo quondam papà ser Jani 288.

Marcopulo I'etro 301.

Marcopulo Zuane 315.

Marcopulo Zuane de papà 315.

Marcopulo Zuane quondam papà 288 ,

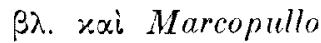

Masarachi Stamatli 313.

Masarachi Zuane, capitano 307, 31'4.

Masrichi Mare'Antonio 319, 323.

Mavrichi Marc'Antonio quondam ser Stamatti 286.

Mavrichi Stamali quondam ser Dimo 286.

Megaduca Dimitri 302.

Melissino Stamatti quondam ser Giacomo 281.

Menaggia Andrea 3:0.

Menaggia Zorzs 321, 322. 
Metaxà (?) A gnandio 288 .

Metaxà Antenoro 295.

Metaxì Antenoro, proveditor alla sanità 276.

Metaxà Francesco 31'.

Metaxà Francesco quondam ser Marc' Antonio 278.

Metaxà Gerolimo quondam ser Costuntin 279.

Metaxà Giacomo 311,314 .

Metaxà Gícomo quondam ser Marc'. Inlonio 278.

Metarà (?) Giorgo 288.

Metaxà (?) Lorenzo 288.

Metaxà Manoli 319.

Metaxà Manoli quondam ser Lucì 280.

Metaxì Marcello 303, 318.

Metaxà Marcello quondam ser Costantin 279.

Metaxà Metaxà $282,295,319$.

Metaxà Petro 28:, 319.

Metaxà Stamatti $310,344$.

Metaxà Stamatti quondam ser Marc' Anlonio 278.

Metaxà Stati de papà Alvise 288.

Metaxà Valiano 312, 31'́.

Metaxà Valiano quondam ser Marc'_1ntonio 278.

Metaxà Zorzi 312 .

Metaxà Zorzi, giudice 276.

Metaxà Zorzi quondam Franco 295.

Metaxà Zorsi quondam (ser) Lucà 285, 295.

Metaxà Zorzi Vecchio 321.

Michalitiano Anaslassi 286, $310,320$.

Michalitiano Teoffilo 286, 311, 319.

Miglaressi 296.

Migliaressi $\beta \lambda$. Miglaressi.

Miliaressi si. rai Miglaressi.

Miliaressi Galeazzo 304, 306.

Miliaressi Galeazzo de ser Zuane 278.

Miliaressi Scipion $311,319$.

Miliaressi Scipion quondam ser Niroló 287.

Miliaressi Steffanin $302,310$.

Miliaressi Steffan in de ser Zuanne 278 .

Miliaressi Zuane 312.

Miliaressi Zuanne, giudlee 276.
Milonà Costantin 279, 305.

Milonà Francesco 306.

Milonà Francesco quondam ser Andrea 279.

Mingardo Anzolo 293, 30\%, 311.

Mingardo Anzolo quondam ser Lunardo 278.

Mingardo Damian 303.

Mingardo Damian quondam ser Lunardo 278.

Mingardo Gasparo, signore alle strade 277.

Mingardo Todorin 296, 304, 311.

Mingardo Todorin quondam ser Lunardo 279.

Mistro Calló Giorgo de ser Nuzzo 290.

Mistro Callò Nuzzo 290.

Montesanto 3ג. De Montesanto.

Moro Antonio 286.

Musugli Jiuglio (Jiulio) $\beta \lambda$. Musuli.

Musule J(i)ul(l) io 286, 295, 301, 311, $319,320, \beta \lambda$. каi Julio.

Vegro Piero $283,293$.

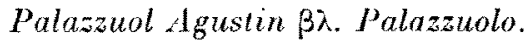

l'alazzuol Agustin quondam ser Piero 277 37. xai Palazzuolo.

Pralazuol Francesco quondam Zam'ser Battista 277.

Palazzuol Marc'Antonio 303, 310.

Palazzuol Mare'Antonio quondam ser Zam'Battista 277.

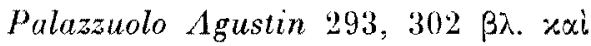
Palazzuol.

Panà Agustin 284.

Pand Anastassi 297.

Punà Antonio 283.

Panà Dario 283, 297.

Panà Demetrio quondam ser Alvise 282.

Panà Dimitri 297.

Pant Francesco 283, 297.

Panà Gabriel quondam ser Michali 284.

Panà Giorgo 297.

Panà Giorgo de Jani 28'́.

Pand Giorgo de ser Marco 288.

Panì Jani 283, 297.

Panà Loiso quondam ser Andrea 284. 
Panà Loyso 283.

Pand̀ Lunardo de ser Francesco 288.

Panà Marc'Antonio quondam ver Steffanin 28 '.

Panà Nicoló 283, 297.

Panà Ptero de ser Todorin 288.

Panà Vizzo 28/.

Panà Zorza de ser Alouse 288.

Pandogalo Andrea quondam ser Gabrel 286.

Pan logalo Alidruzzo 298.

Pandogalo Bello quondam ser Cabrel 286.

Pandogalo Gabrlel de ser . Indrea 286.

Pandogalo Manoli 284, 295, 298.

Pandogalo Orlando quondam ser Leo 28 '.

Pandogalo Timotheo 299.

Pandogalo Timothio quondam Manole 282.

Papateropito Stamatt 284

Pecator Nicolò $3 \lambda$. Peccator.

Peccator Agustin 30 t

Peccator Agusitn quondam ser Lorenzo 284.

Peccator Alessandro 299

Peccator Alessandro quondam ser Nicolò 286.

Peccator Antonto 294, 302, 312

Peccator Antonto de ser Galeazzo 277.

Peccator Domenico quondam ser Lorenzo 284 .

Pecrator Galeacio (Galeazzo, Gallazzo), sindlco $\langle 274\rangle, 275,276,291,293$, $297,299,301,303,304,306,\langle 307\rangle$, $\langle 308\rangle, 309,312,314,315,316,\langle 317\rangle$, $318,320,321,\langle 322\rangle, 323$.

Peccator Marco 303.

Peccator Marco quondam ser Lorenzo 284.

Peccator Nicolo 302, 308, ambasciatore 309, 316, 〈322〉 $\beta \lambda$. xxi Pecrator Vlsconte.

Peccator Nicoló de ser Galeazzo 277.

Peccator Stamattelo 302.

Pecrator Zan'Domenego 306

Peccator Vusconte Vicolo, ambasciatore

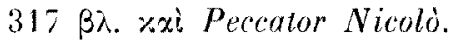

Pelerudi Andrea 302, 312.

Perlengin Andrea 283, 299.

Perlengin Gerolimo 305

Perlengin Gerolimo de ser Mathio 283.

Perlengin Manoli 283.

Perlengin Matho 283. 298

Polcalì Antonio 298.

Policala Antomo quondam ser indrea 284.

Poliralà Battista $287,299$.

Polcalà Llia 287.

Policalà Giorgo 285. 298.

Policala Marmo 287.

Policalò Nicoló de ser Girorgo 285.

Policalà Priamo 299.

Polıcalà Priamo quondam ser 1ndrea 284.

Poltcalà Zuane de ser Grorgo 287.

Raftı. 1ndrea 296,304 .

Raft. Andrea quondam ser Stamatt 280.

Renessi Gunt 322.

Rizzardopuli $\beta \lambda$. Rizzardopulo.

Rizzardopullo Dimo 295.

Rizzardopullo Paollo de Januzzo 311.

Rizzardopulo Achlle 294, 310, 31'.

Rizzardopulo Achlle de ser Costantin 288

Rizzarlopulo Alessandro 310.

Rizzardopulo .Inastass quondam papà 288

Rızzardopulo Aurelıo 311.

Rizzardopulo Dimo quondam ser Stamatte 288 .

Rizzardopulo Francesco 310.

Rizzardopulo Francesco do ser Antonı 283.

Rizzardopulo Francesco quondain Antono quondam papà 282.

Rizzardopulo Francesco quondam 1ntonio quondam Tomà 297.

Rizzardopulo Franresro de papà 296.

Ruzzardopulo Francesco de papà ser Domuncco 286.

Rizzardopulo Franglà 315.

Rızardopulo Jan de ser Andrea 287.

Rizzardopulo Lurcì 283 .

Rizaardopulo Marro 283, 302. 
Rızardopulo Mechale $31^{\prime}$

Rizzardopulo Michel 294, 310.

Rizzardopulo Miehel auondam Jane 287 .

Rizzardopulo Nadal 295, 315

Rizzardopulo Nadal quondam ser (ostantin 286

Rizzardopulo Nicoló 283, 29', 315.

Rizzardopulo Necolo quondam indrea 297

Rizzardopulo Vicolò quondam Ianı 287.

Rizzardopulo Paollo quondam ser Januzzo 286.

Rizzardopulo Pvero 315

Risardopulo Plero quondam Jani $28 \%$.

Ruzzardopulo Rel(l)o 296, 315.

Rizzardopulo Relo de papíser Domunico 286.

Rizzardopulo Simo 315.

Rtszardopulo Simon quondam Jane 287.

Rızzardopulo Todorin 283

Rızzırdopulo Tomà 283.

Ruzardopulo Zuane 28:3

Rizardopulo Zuane quondam indrea 297.

Roccoler Vener 302, 308.

Rocroler Venzer quondam ser Zane 278.

Rossolmo Antonio 283.

Rossoltmo Cumıno 299.

Rossoltmo Cristodulo 283.

Rossolımo Dimitr 299.

Rossoltmo Giacomo 283

Rossolemo Zuane quondam messer . Mario 283, 297.

Rossolimo Lafhani Intonio 297.

Salamon 1lstse 288.

Salamon Francesso 304.

Salamon Francesco de ser Mathio 278.

Salamon Lorenzo 288, 320 .

Salamon Mathio 302, 30 r.

Salamon Matho fu del nobilhomo Gacomo intonio 277.

Salamon Sebastıan 288

Salamon Tiberio 288.

Salamon Velissario 288.

Salamon Virenzo $3123 \lambda$. xat solomon.

Salamon Vicenzo de er Mathı 278.

Salamon Zorsi 288, 32ㄴ.
Salamone $\beta \lambda$. Salamon.

Sarachunopulo Zan'Francesco 305.

Schradà Petro 310. 313.

Schradà Zuane 295, 311, 313.

Schtadà Zuane de ser Petro 285.

Sdrigna Dardo 305.

Sdrigna Odoardo quondam ser Lucà 279.

Sdrigna Zuane 279

Servò Battista 298.

Servò Battusia quondam ser Stamatt 280.

Servo Dimitr 298.

Servo Dimitri de ser Teoffilo 280

Servó Draco de ser Teofflo 280

Servò Francesco de ser Teoffilo 280.

Serso Franco 299.

Servo Gerolimo de ser Zuane 280

Servò Lunardo 29', 304.

Servo Lunardo quondam ser Tenfflo 279.

Servò Marco 298.

Servo Marco de ser Teoffilo 280

Servò Petro de ser Zuane 280

Servó Simon de ser Zuane 280.

Servó Teoffilo 299.

Servò Teoffilo quondam ser Giorgo 280.

Servo Zuane quondam ser Glorgo 280.

Siclian Benetlo $\beta \lambda$. Siculiano

Sicilian Francesco 305.

Siculian Cieroltmo $3 \lambda$. Stevluano.

Sicrlano Benetto 288, 306.

Simllano Gerolımo 288, 306.

Solomon Vtrenzo 303 3\%. xal Salamon

Soymert Manoll 321.

Spata Giorgo 321.

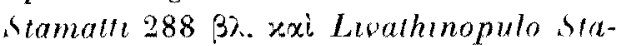
matil.

Stoppa Benetio 306.

Stoppa Benetto, bombardier 287.

Strambali Giacomo (Jaromo) $277,302$.

Stravaletra (?) 288.

Sult Giorgo quondam ser Janı 285.

Surlano Giaromo 279, 293, 306.

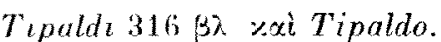

Tipaldo 298 .

Tipaldo Alessandro 294, 299, 316.

Tipaldo Alessandro fu di ser Antonio $\pm 81$

Tipaldo Andrea 29'. 
Tipaldo Andrea fu de ser Antonio 281.

Tipaldo Andruzzo 298, 316.

Tipaldo Antonio quondam ser Paolo 281.

Tipaldo Apostoli quondam ser Costan$\operatorname{tin} 281$.

Tipaldo Costantin 316.

Tipaldo Costantin fu de ser (quondam) Antonio 281, 299, 316.

T'ipaldo Costantin de Draco 294.

Tipaldo Costantin quondam (ser) Draco $281,298$.

Tipaldo Dimetri (a) 294, 299.

Tipaldo Dimitri $(\beta) 294$.

Tipaldo Dimitr quondam ser Draco 281 , 298.

Tipaldo Dimutri quondam ser Marco 281.

Tipaldo Draco 294, 316.

Tipaldo Draco de ser Paolo 281, 299.

Tipaldo Frangià (a) 294 .

Tipaldo Frangià ( $\beta$ ) 294 .

Tipaldo Frangià quondam ser ¿Indrea 281.

Tipaldo Frangià quondam (ser) Marco $281,298$.

Tipaldo Janachi 316.

Tipaldo Jani d'Arseni 294.

Tipaldo Jant quondam ser Arseni 281.

Tipaldo Jani de ser Galeazzo 281.

Tipaldo Janicarhi 299.

Tipaldo Jorio 299.

T'ipaldo Lentulo 316.

Tipaldo Lentulo de ser Paslo 281, 298.

Tipaldo Leono 294.

Tipaldo Leono de ser Lambrinò 281.

Tipaldo Lanardo quondam ser Carlo 281.

Tipaldo Manoli de ser Nicolò 281.

Tipaldo Marco quondam ser Arseni 281.

Tipaldo Nicolò quondam ser Marco 281.

Tipaldo Nicolò quondam ser Paslo 281.

Tipaldo Pavlo 294, 299.

Tipaldo Pavlo quondam ser Nicolo 281.

Tipaldo Piero 294, 299, 316.

Tipaldo Piero de ser Lambrinò 281.

Tipaldo Stamattelo quondam ser Velissario 281.

Tipaldo Stamatti 311, 316.

Tipaldo Stamatti de ser Nicolò 281.
Tipaldo Steffanin quondant ser Giacomo 290.

Tipaldo Tomà 294, 298.

Tipaldo Tomà fu de ser Antonio 281.

Tipaldo Vardaramo de ser Nicolò 281.

Tipaldo Velissario quondam Petro 291.

Tipaldo Zorai ( $\alpha) 316$.

Tipaldo Zorzi (B) 316.

Tipaldo Zorsi de misser Draro 294.

Tipaldo Zorzi quondam ser Draco 281 , 299.

Tipaldo Zorzl de ser Nicoli 281.

Travlò Alessandro 311.

Travlò Alessandro de Manoli 313.

Travlò Alessandro quondam ser Manoli 286.

Travld Andrea 295.

Traolò Indrea de stratloto 311.

Traolò Andruzzo de (ser) Nrolò 286 , 313.

Travlò Andruzzo de (ser) Stamatti 285, 313.

Trasló Apostoli $310,313$.

Traolò A postoli quondam Federigo $\geq 8:$.

Travló Barbarigo 313.

Traoló Barbarigo quondam ser Manoli 286.

Traoló Cosnà 296, 313.

Traolò Cosmà de Draco 311.

Traolo Gabriel 284, 311.

Travlò Gabriel quondam Manoll 294.

Traoló Gerolimo ( $\alpha)$ 295, 311.

Traolò Gerolimo ( $\beta$ ) 312 .

Travlò Gerolimo de (ser) Tunardo 284 , 313.

Travlò Gerolimo de Manolı 313.

Travlò Gerolimo quondam Manoli 295.

Travlò Giacomo, giudice 276.

Travlò Giacomo d'Andrea 296, 313.

Travlò Giacomo quondam Andrea 310

Traslò Giacomo de Manoli 311, 313.

Travlò Giacomo quondam Manoli 296.

Traslò Giacomo quondam ser Michali 286, 311.

Travlò Giorgo d'A postoli 311.

Travld Giorgo quondam (ser) Apostoli $286,313$.

Traolò Jani 296, 310. 
Traolò Jani de Pavlo de Stelo 313

Travlò Leo 313.

Travlò Laceda 313.

Travlo Lunardo $294,310,313$.

Travlo Lunardo quondam ser Hichali 28's'

Travló Marco 295, 310.

Travlò Marro de Lucà 313.

Travlo Marco de Lunardo 311.

Travlo Marco de (ser) Zuant 286, 313.

Travlò Nicolo 295.

Traslò Nicolo, capltano al Theachi 27.2.

Trawli Nicolo de (ser) Leo 285.310 , 313.

Travló Nicolò de Stamatii 311.

Truplò Oratio de (l) papà 285, 296, 313

Travlo Stamatt de Federico (Federigo) 295, 307. 310, 313.

Traolò Stamatti Vechio 312, 313.

Traulò Tomaso 285, 310,313.

Traolo Velissario 296, 313.

Travlo Zaccaria 295, 312, 313.

Travlò Zorzi 295, 314.

Travlò Zorzl de (ser) Luc(cì) 285, 307.

Travli Zuane 295.

Travlo Zuane d'A postole 296, 313.

Travlò Zuane de Tomaso 313.

Trech Bernardın 305, capttano 285, 293.

Trechl Bernardin, rapitano $3 \lambda$ Trech.

Valianl $3 \lambda$. Valiano.

Valiano Andruzzo 315.

I'aliano Andruzzo quondam papà ser Giorgo 286.

Valiano Apostoli 315.

Valiano Apostoli quondam papà ser Marco 287.

Valiano Costaniin $295,315$.

Valtano Costantin quondam papà ser Marro 287.

V'alıano Cristofforo 295, 310, 31 '́.

Valiano Cristofforo quondam ser . Lntonio 288.

Valiano Dimitri d'Antonio 31.9.

Valıano Dımo 295, 310, 315.

Valiano Dimo de papà 315 .

Valiano Dimo quondam papè ser Marco 287 .
Valuano Geroluno 29', 315.

Valıano Giacomo 295, 315.

Valıano Jant del Calogero 315.

Valiano Marco 314.

Valiano Nicoló 314 .

Valiano Nicolò quondam ser Zaccaria 285.

Valiano Stamatti 315.

$V$ aliano Stamatt de papà 295.

Valiano Zaccarta 295, 315.

I aliano Zaccaria d(e ser) Antonio 286 , $312,315$.

Valuano Zaccaria de papì 282, 312

Valiano Zaccaria quondam papà ser Marco 287.

Valter Antonio 293, 302, 308.

Valuer Antonio quondam ser Zuane 277.

Valsamachi Anzolo 297.

Valsamachi 1 nzolo quondam ser Ptero 285

l'alsamachi Colela 297.

Valsamach Colela quondarn ser Piero 285.

Valsamachi Polo quondam ser P'ero 285.

Valsamachi Zorzi 297.

Valsamach Zorzi quondam ser Piero 285 .

Vasilopulo Nicoló 310.

Vergotun Costantun 295, 321.

Vergotin Costantin quondam ser Vergotin 285.

Vergutan Nicoló 319.

Vergutin Nicolò quondam ser Marco 289

Vilaraut Federigo 289, 305.

Vunari Apostoll (Apostolo), caputano $280,319$.

Vinari Cesare 321.

Vinari Cesare quondam ser Jani 282.

I'inari Demetro (Dimutri) capitano $280,319$.

Vinari Zorzi de capitano 280,321 .

Y isentin Andrea quondam ser Zuane 278.

Visentun Marc'. Intomo quondam ser Zuane 278.

Visentin Zuane 302.

Visentin Zuane, coadiutore 310.

$V$ isentin Zuane quondam ser Marc' $\mathbf{A n}$. tonio 278. 
Visentun, $3 \lambda$. Visentin.

Volterra Francesco 294, 303.

Volterra Francesco fu de ser Todorin 277.

Volterra Gulielmo 294, 302, 310.

Volterra Gulielmo quondam ser Cesare 277.

Volterra Nadal 303.

Volterra Nadal de ser Stamattı 277.

Volterra Nicolò 304, 311.

Volterra Nicoló quondam ser Cesare 277.
Volterra Stamalil 293, 302, 310.

Volterra Stamatti quondam ser Arsem 277.

Volterra Zorzl 294, 303, 308, 310.

Volterra Zoral de ser Stamattı 277.

Volterra Zuane 312.

Volterra Zuane, provedtor alla santà 276.

Xomeritena Stamo 318

Zombo Stamattelo 288, 306.

II. $\mathrm{T} O \Pi \Omega \mathrm{N}$

Asso 317 .

Borgo 291, 293, 301, 303, 305, 306

Castello 291, 293, 301, 303, 305, 306, 316.

Cheramiés 293, 31', 315.

Lachitres 293, 319, 320, 323.

Lisurt 298.

Metaxata 319, 320,323.
Palichí 293.

Pesada 293, 297

Spartea 293, 297.

Talamies 293, 300, 301.

Thearhe 277.

Travlata 293, $307 \quad 309,313$.

Venetilt $\langle 275\rangle, 292$

Lante 318.

Zante, città del 318. 


\section{IIIN IKE TENEA $A O T I K O I$}

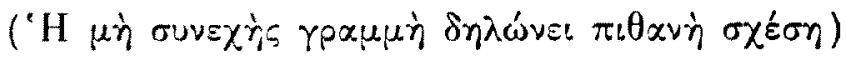
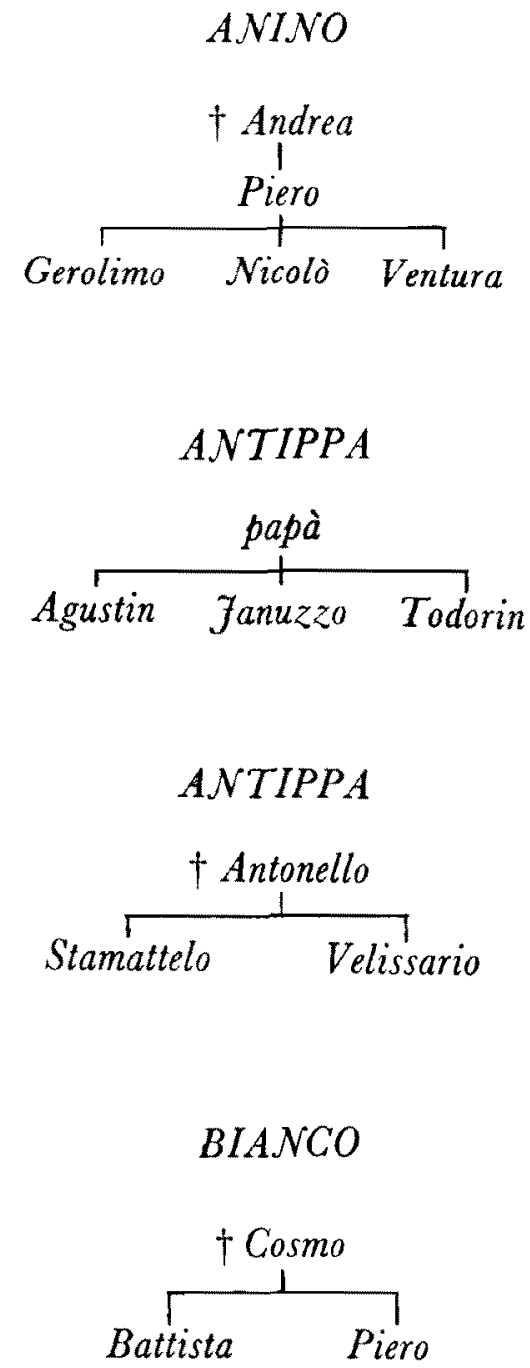

$$
\begin{aligned}
& \text { CAMBICI } \\
& \text { Alessandro } \\
& \text { Giorgo Lazara }
\end{aligned}
$$




\section{CAMBICI}
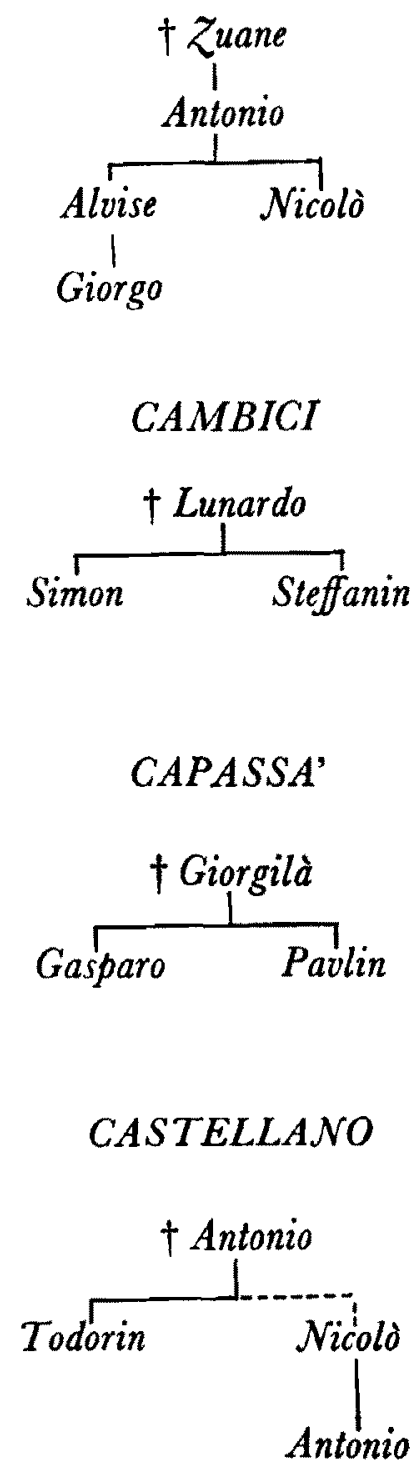

COCCOLI

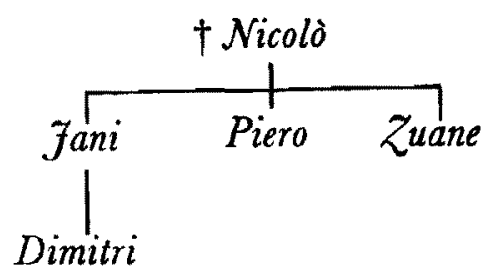


COLOMBIN

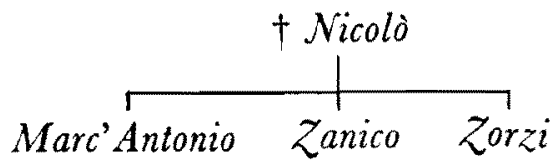

COMBOTECRA

Andrea Todoro

CORAFFA'

$\underset{\text { Hettor Marco Zanetto }}{+}$

CORCUMELI

Gasparo Gerolimo Marc'Antonio Zuane 


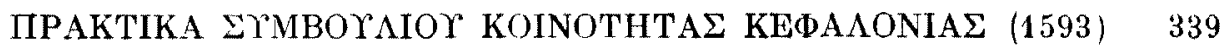

\section{CORCUMELLI}
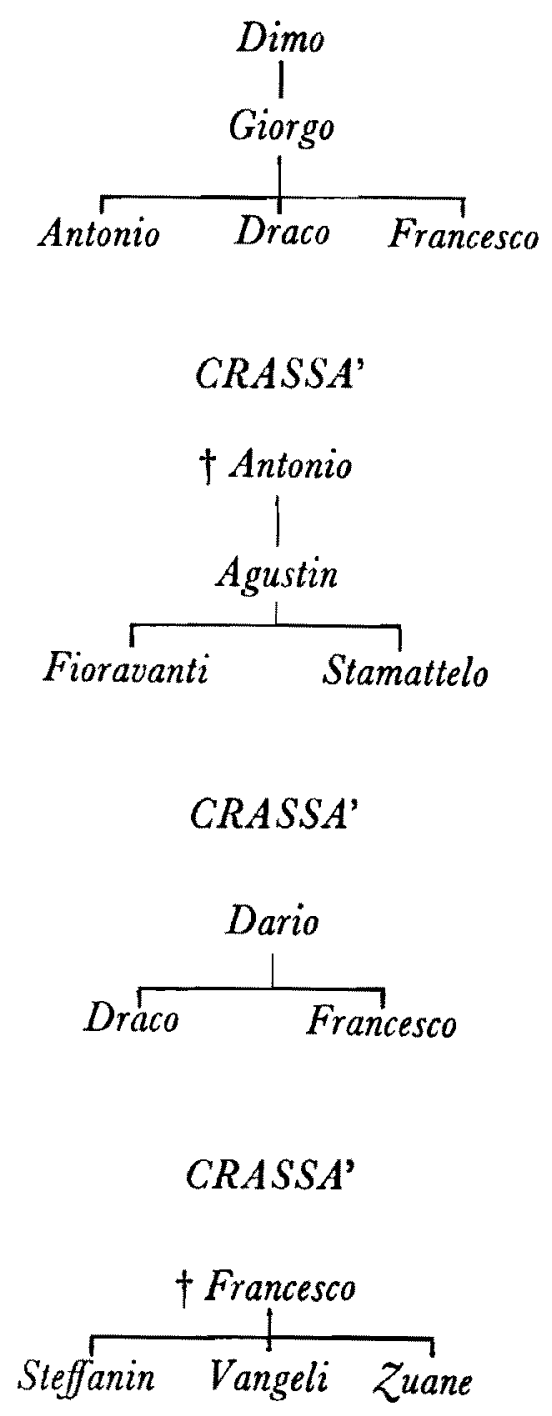

$C Y M A R A$

$\overbrace{\text { Antonio }}^{+ \text {Micolò }}$




\section{CYMARA}

Stamattelo

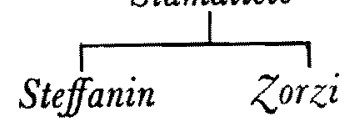

DALLA PORTA

+ Agustin
Alessandro Zuane

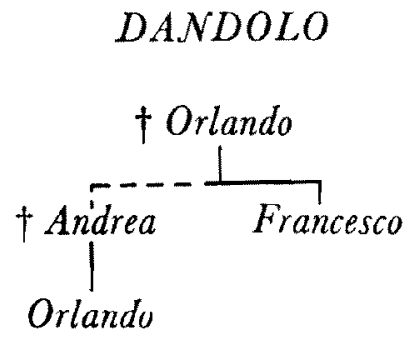

DE MONTESANTO

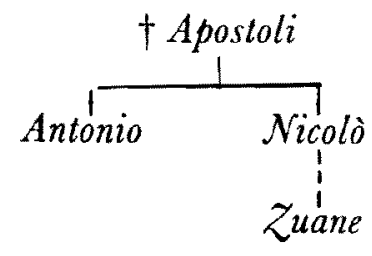

DE MONTESANTO

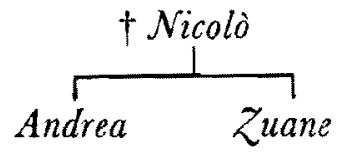




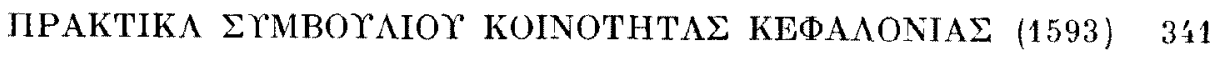
DE MONTLSANTO

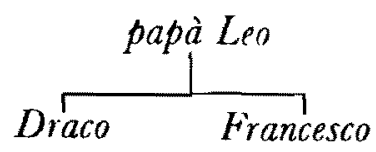

DE MONTESANTO

Andruzzo Gerolimo Jani

DE MONTESANTO

$\underset{\text { Steffanin Stamatti }}{\text { Zuane }}$

DE MONTESANTO

$\uparrow$ Vardaramo

I

Antonio

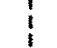

Vardaramo

DE MONTESANTO

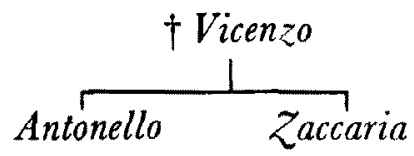

DIACRUSI

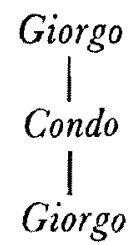


N. I. IIOZXONA

\section{FIOCCA}

+ Mathio

Andrea

Mathio, censor

FUCA'

Anastassi Gabriel Hellor Steffanin

FLCCA'

$\dagger$ Galea $\approx 0$

Andrea

Galea $=0$

FCCC'A'

Fillippo

$\overbrace{\text { Antonio Giorgo vicolo }}^{\text {Fillopo }}$

FICC.A'

$\uparrow$ Frangià

Antonio

Cristodulo Frangia Steffanin Todossi

FICCCI'

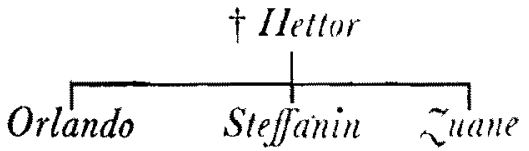




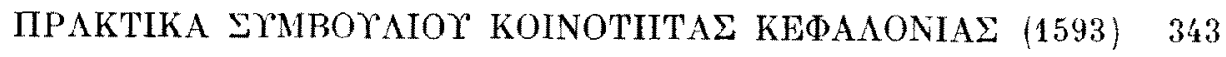

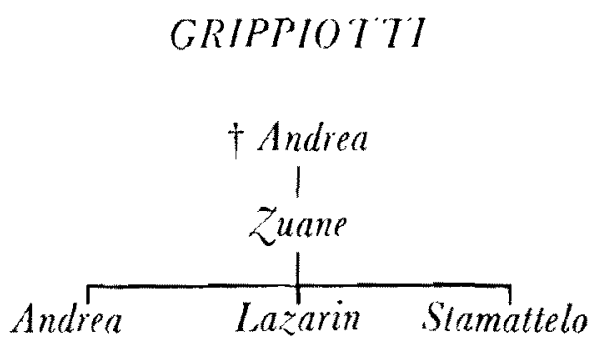

MAGULA'
$+\overbrace{\text { Achilea }}^{+ \text {Nicoló }}$

MARCHETTO

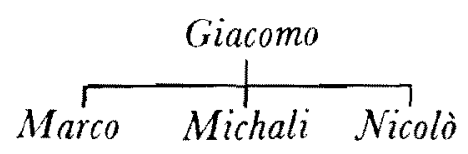

MARCOPUL (L)O

Nicolò $\stackrel{\text { papà }}{\perp}$ Zuane

MARCOPUL (L)O

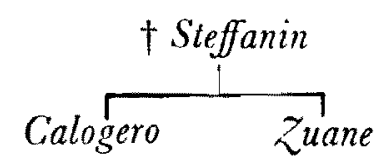

METAXA'

$\overbrace{\text { Gerolimo Costantin }}^{+ \text {Marcello }_{\text {L }}}$ 


\section{METAXA'}

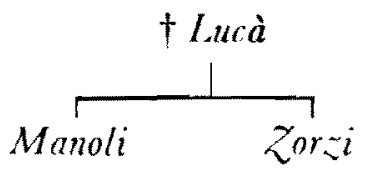

METAXA'

$\overbrace{\text { Francesco Giacomo }}^{+ \text {Marc'Antonio }}$

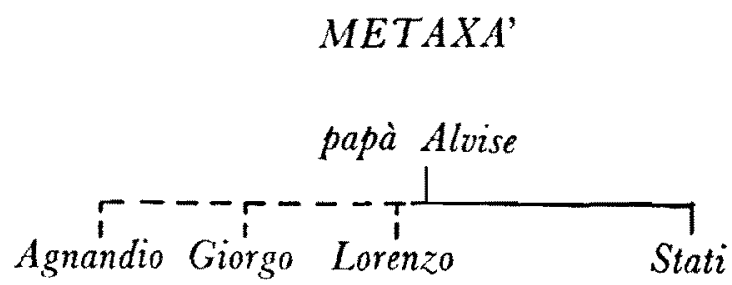

MILIARESSI
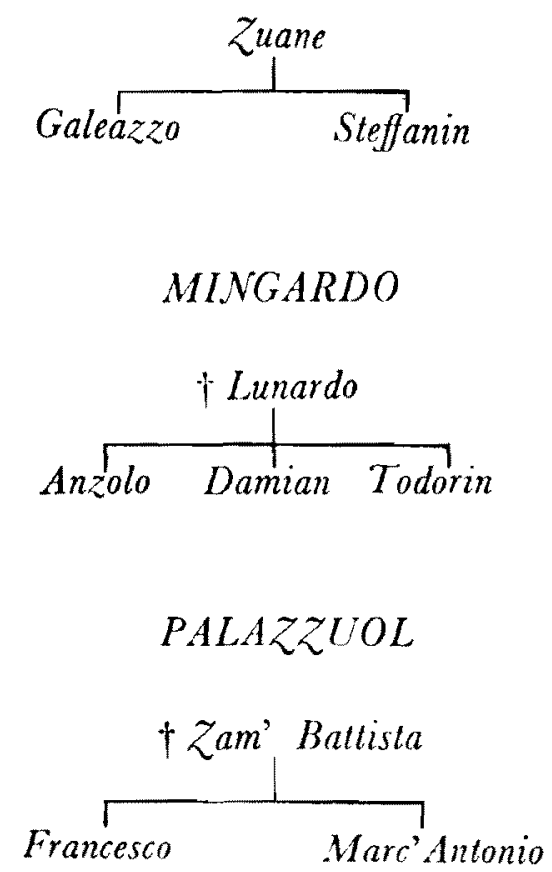


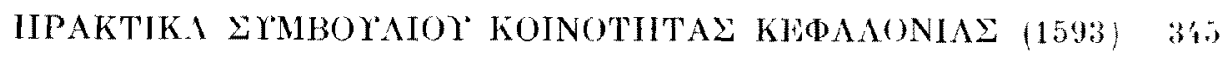

PANDOGALO

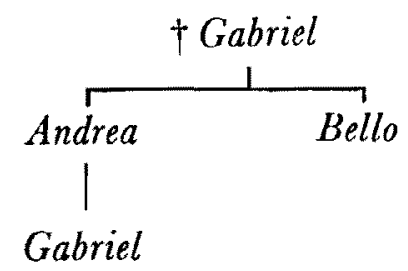

PECCATOR

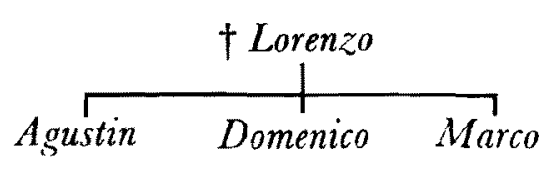

PECCATOR
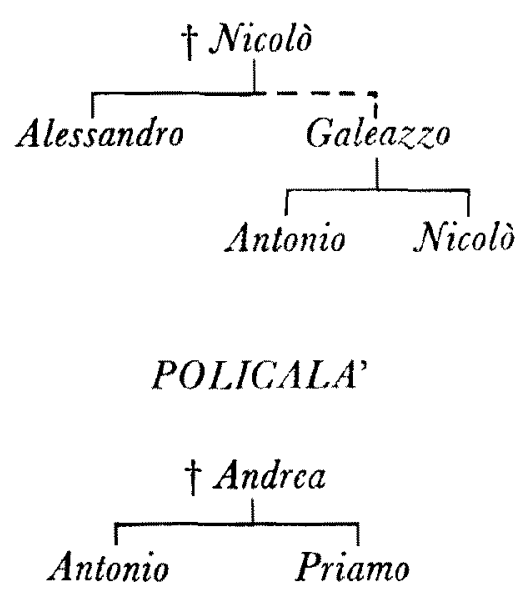

POLICALA'

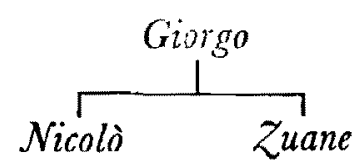


RIZZARDOPULO

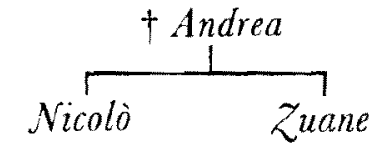

RIZZARDOPULO

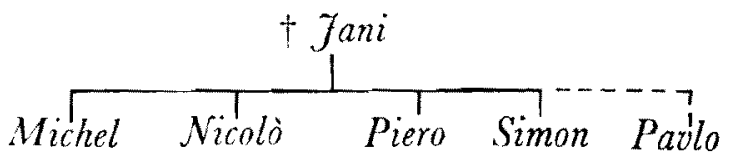

RIZZARDOPULO

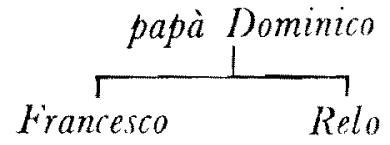

$S A L A M O N$
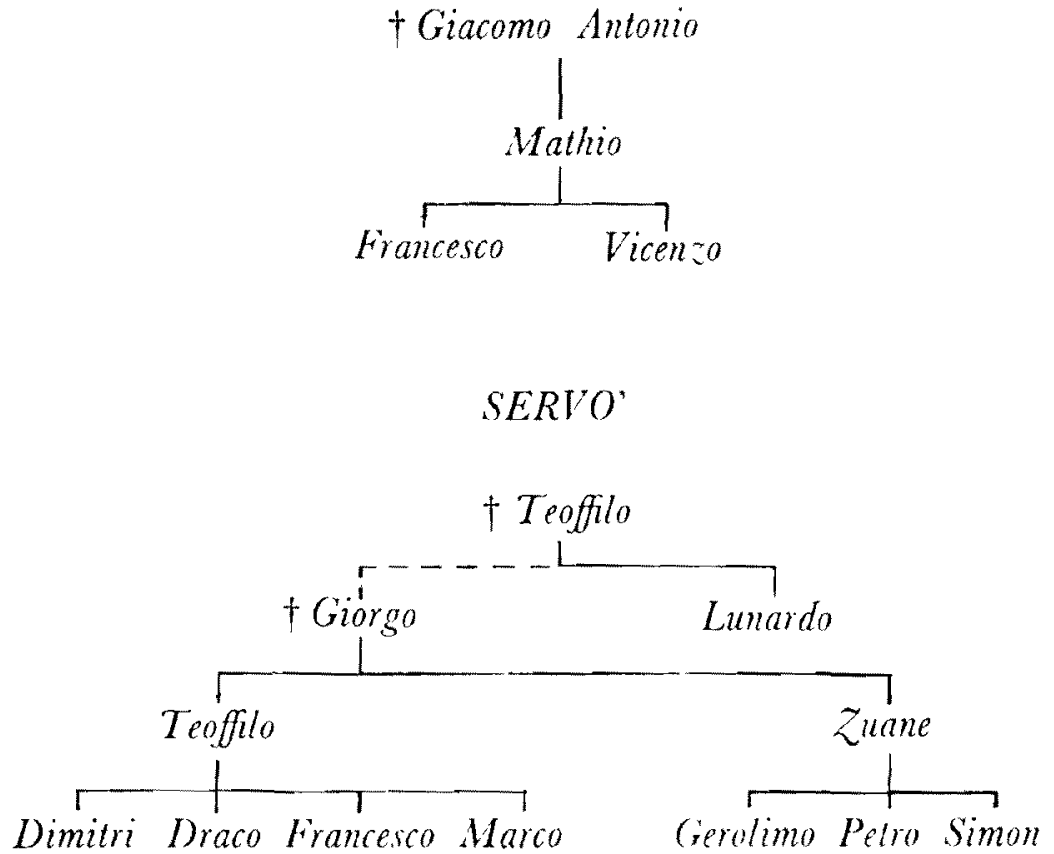
TIPALDO

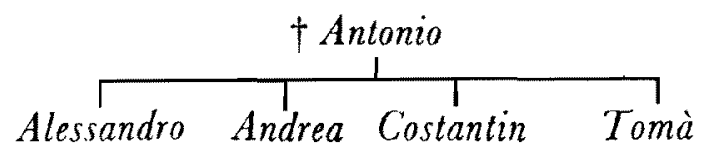

TIPALDO
TIPALDO
+ Mari ${ }_{\text {Draco }}^{+ \text {Arseni }}$
Costantin Dimitri Zor $i$

TIPALDO

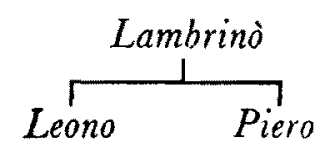

TIPALDO

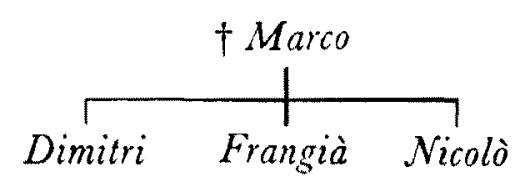

TIPALDO

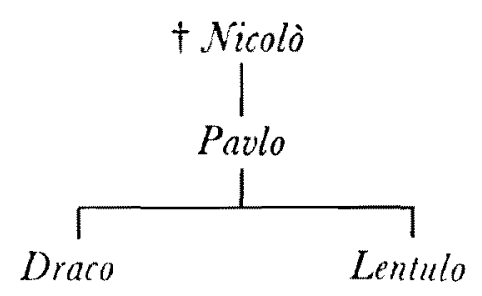




\section{TIPALDO}

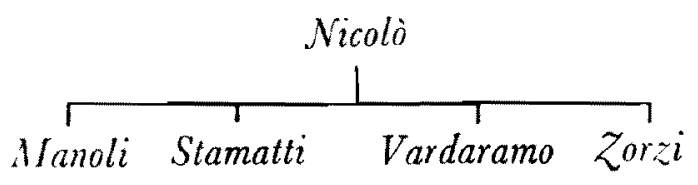

TIPALDO

$\overbrace{\text { Antonio }}^{\dagger \text { Nicolò }}$

TRAVLO'

Andruzzo Nicolò

TRAVLO'

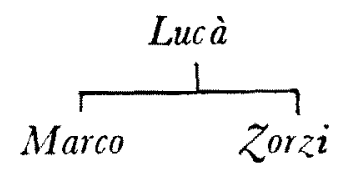

TRAVLO'

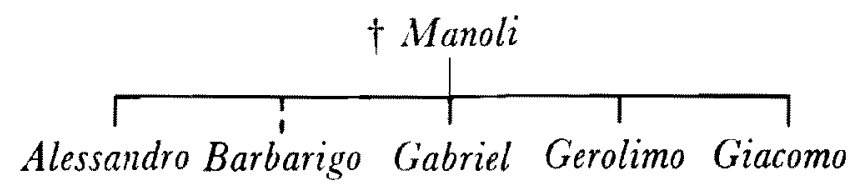




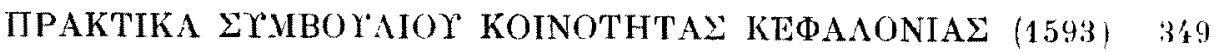

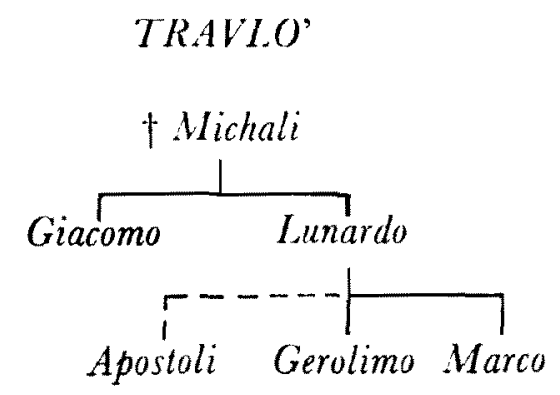

'TRAVLO'
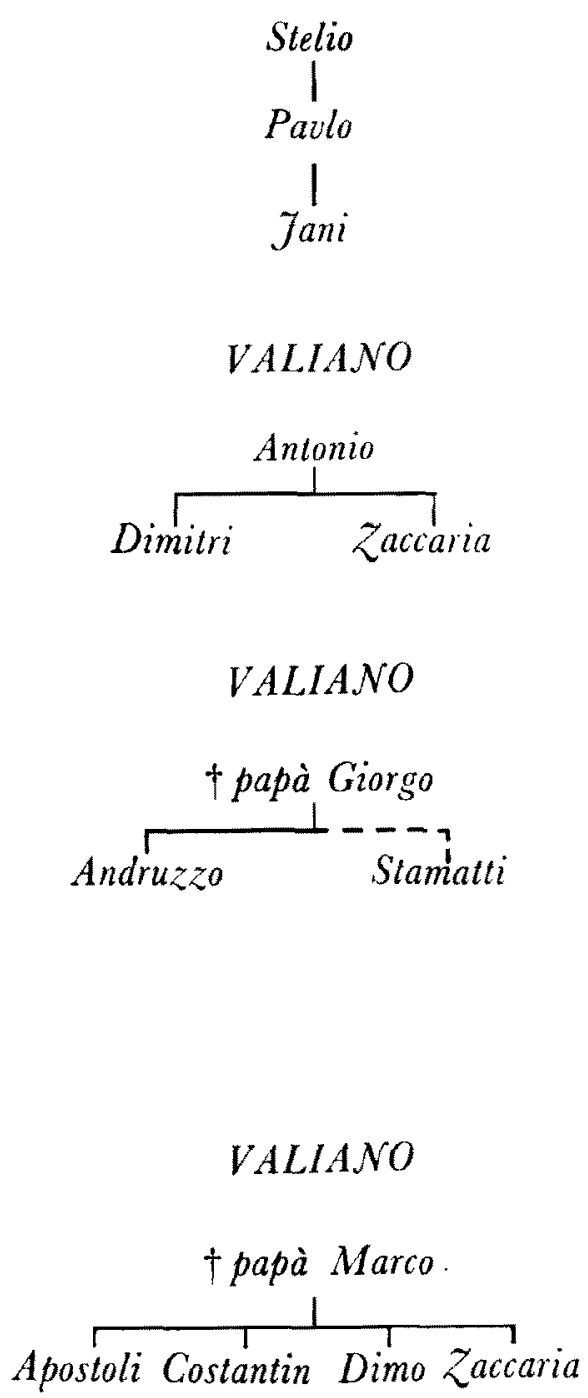
350

VALSAMACHI

$\overbrace{\text { Anzolo Colela Polo Zorzi }}^{+1}$

VISENTIN

$\overbrace{\text { Andrea }}^{\frac{\uparrow \text { Juane }}{\text { Marc'Antonio }}}$

VOLTERRA

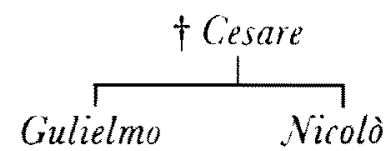

VOLTERRA

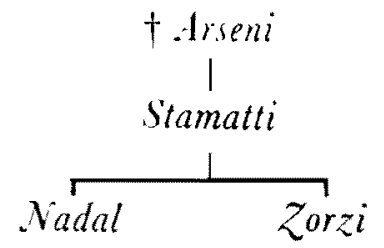




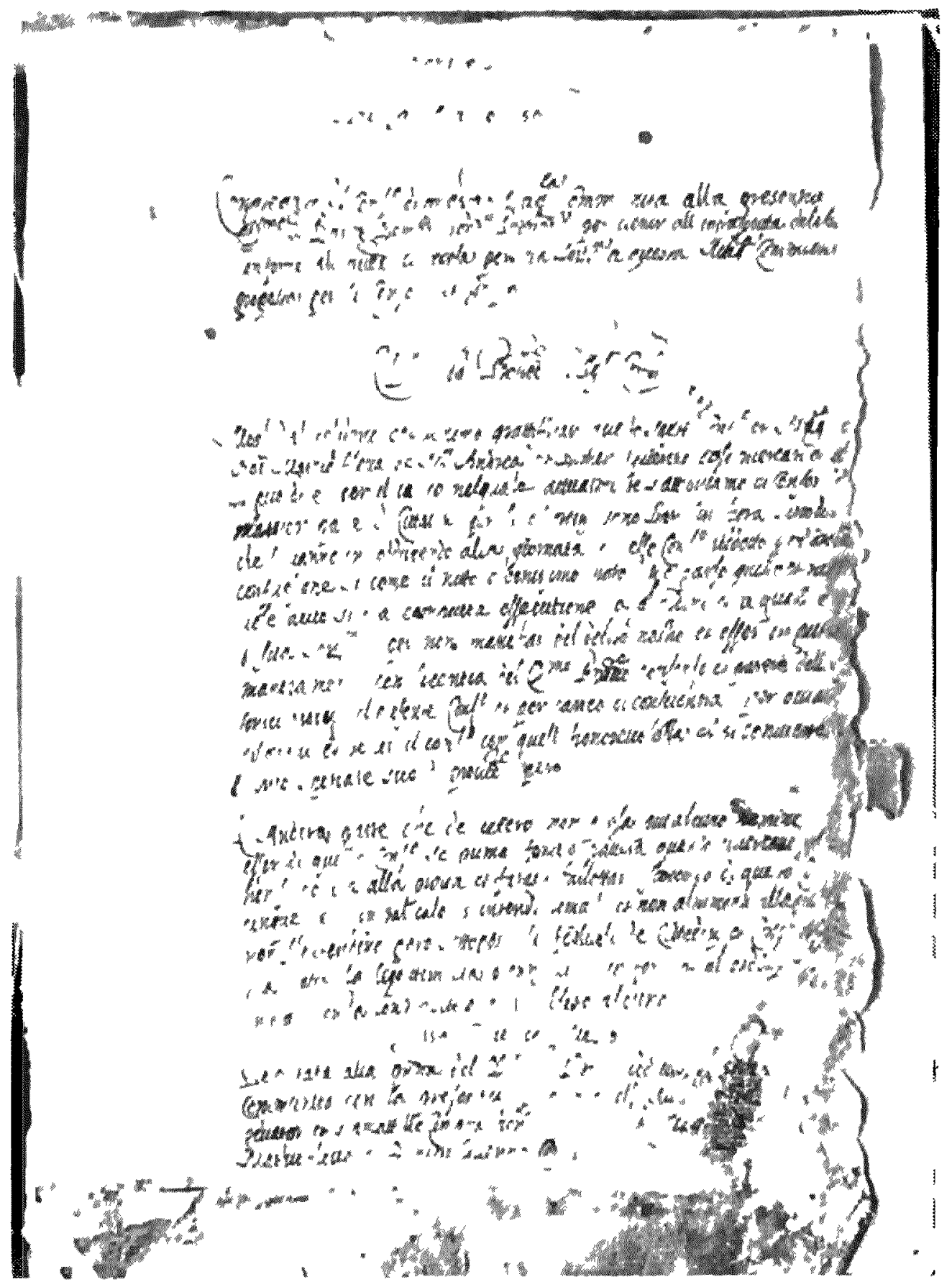

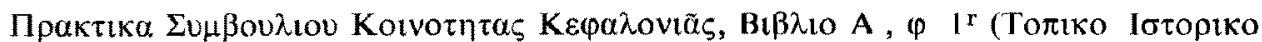

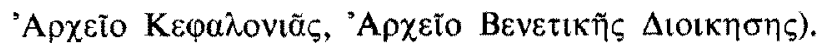


חív. 22

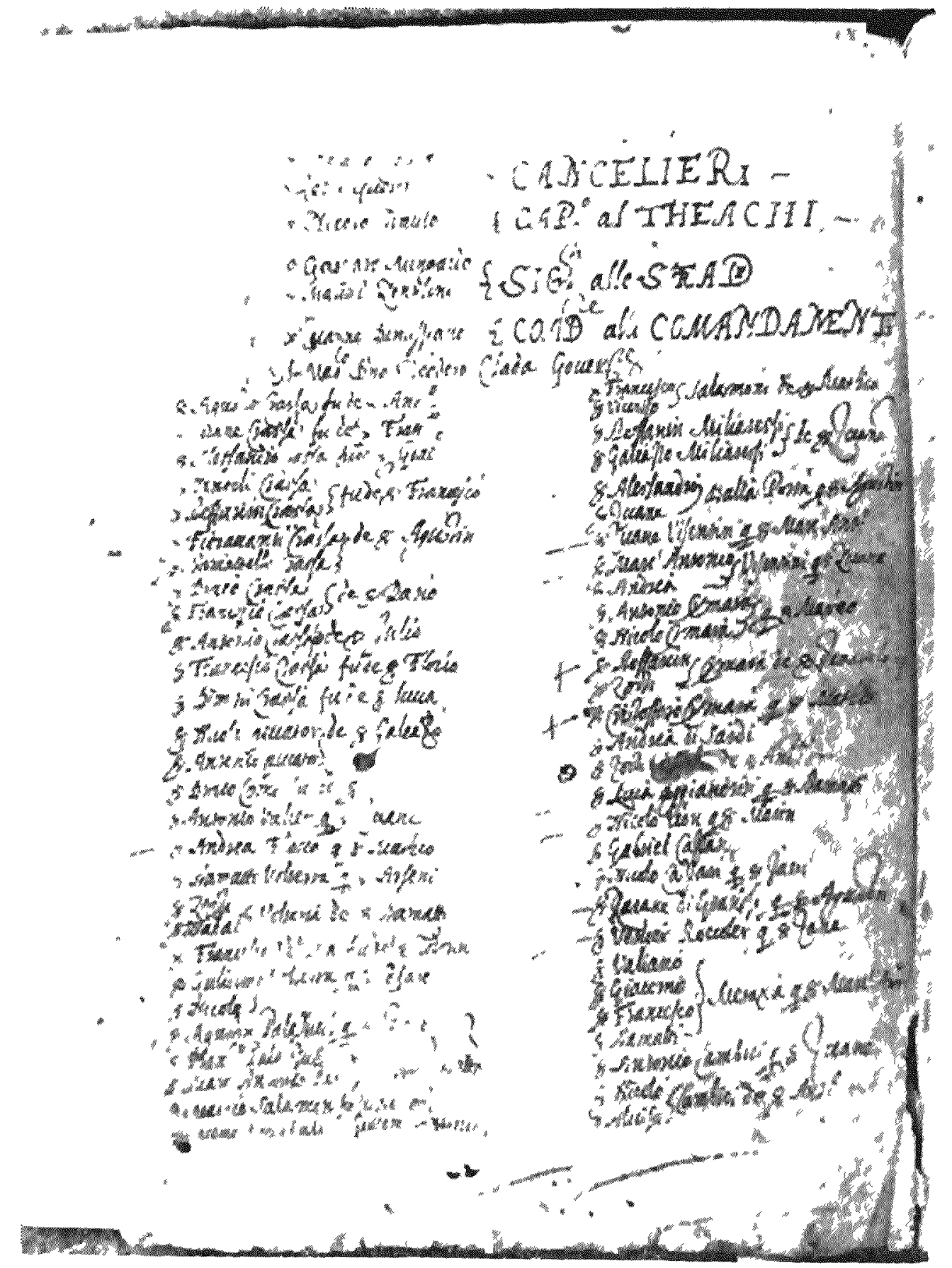

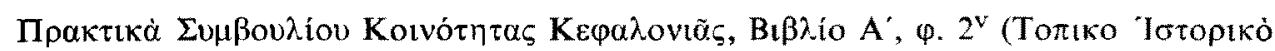
'A 


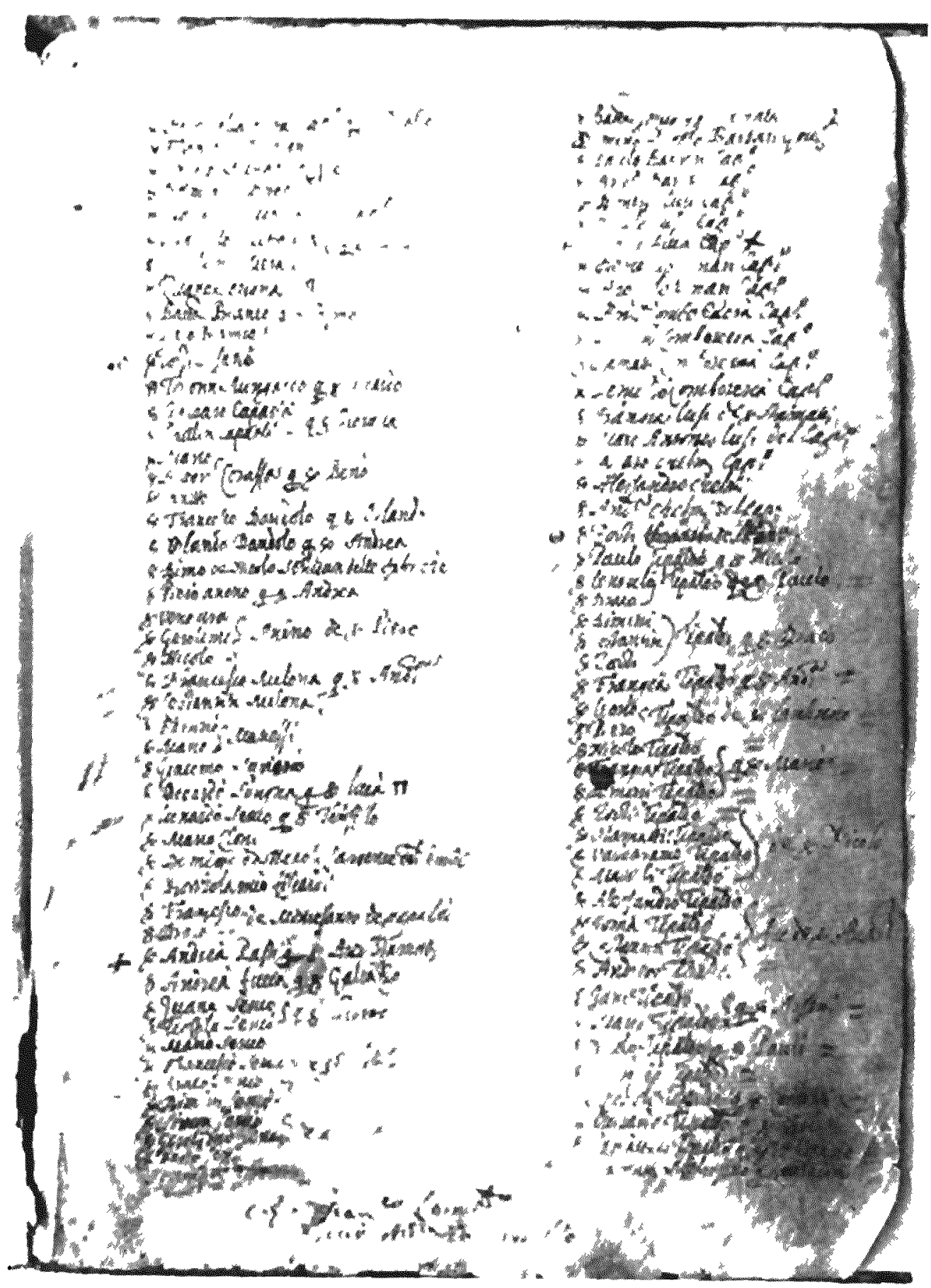

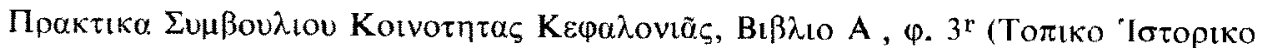

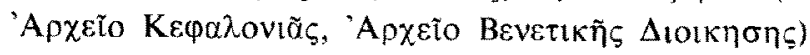


ก1v 24

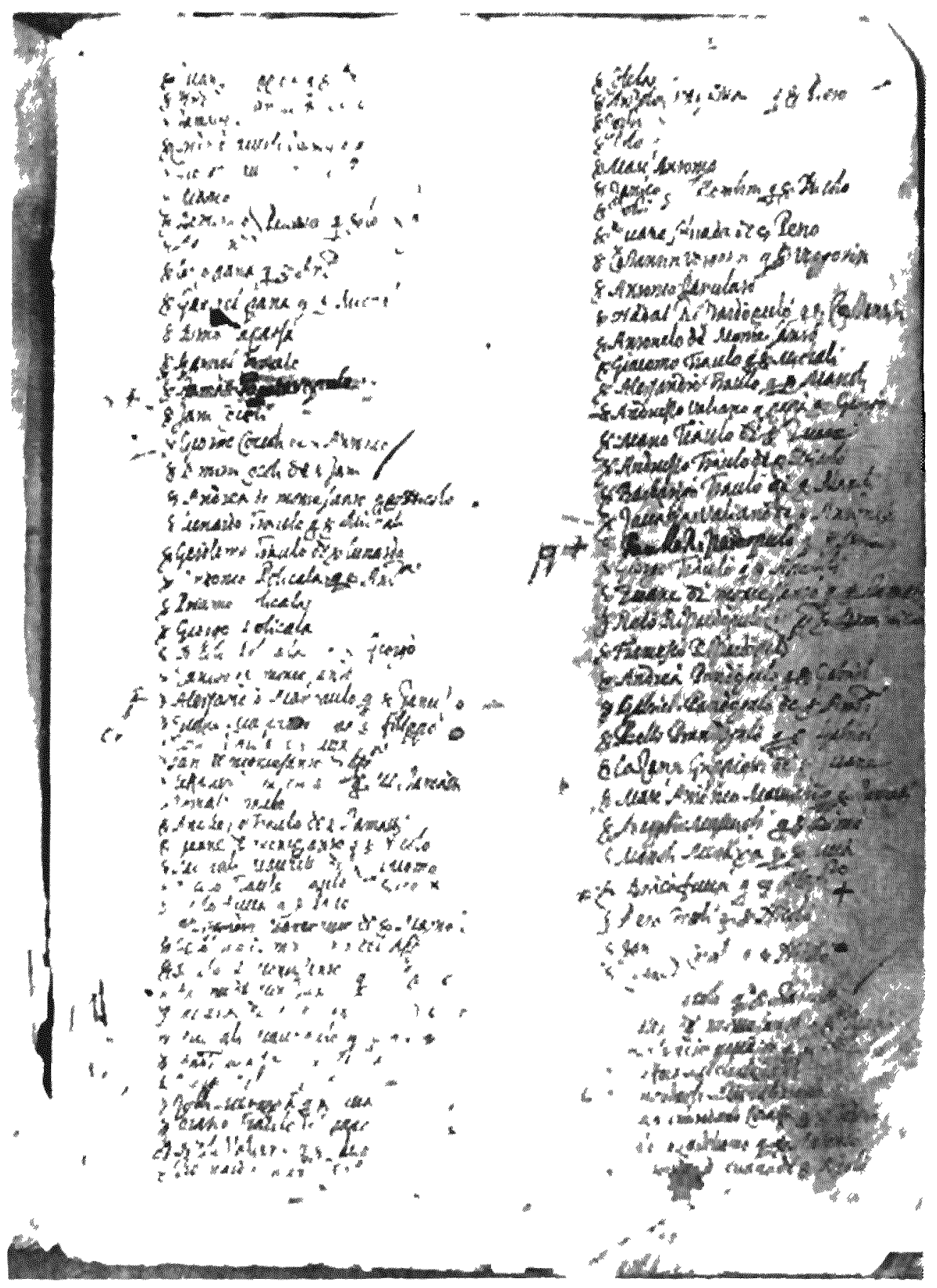

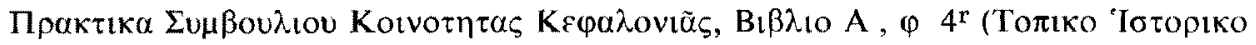

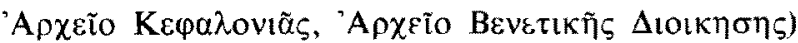




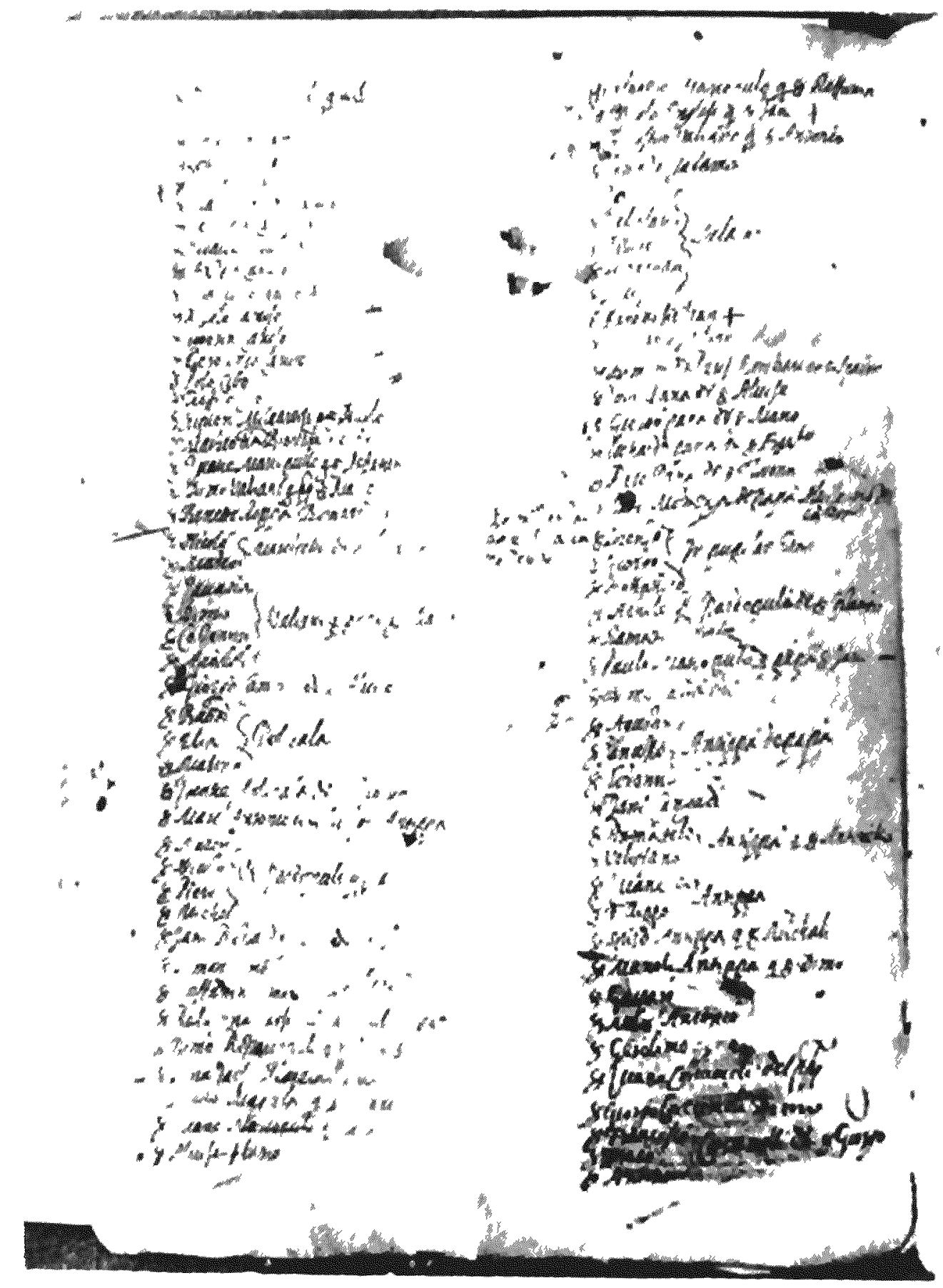

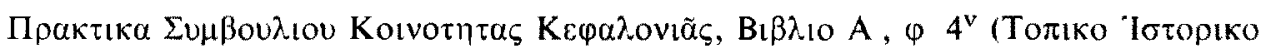

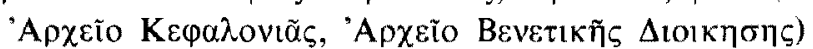


Mı 26

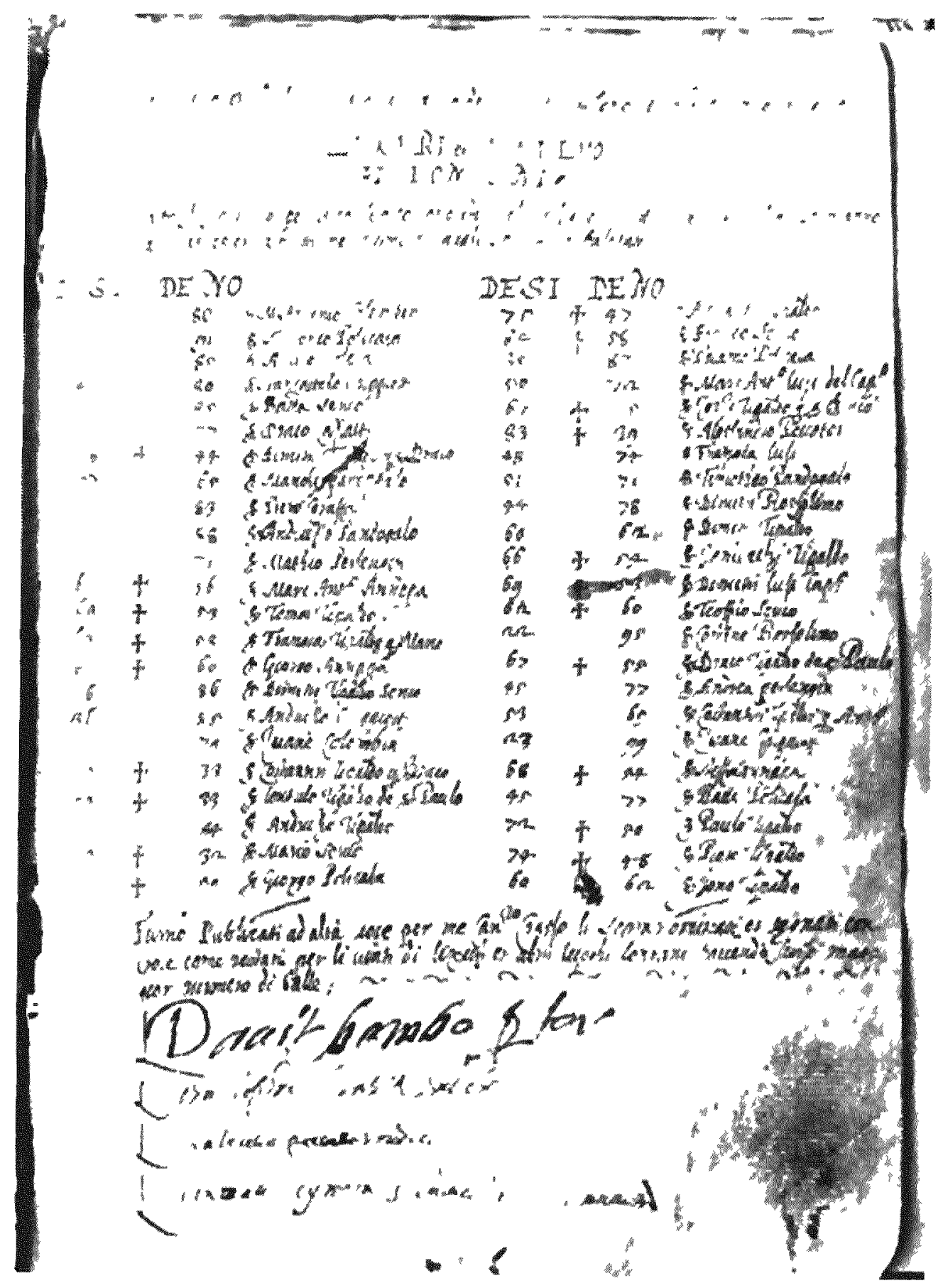

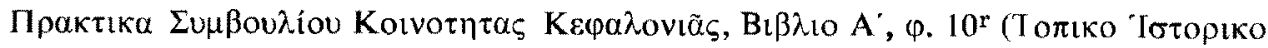

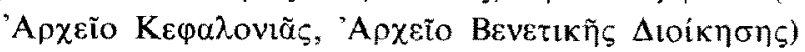




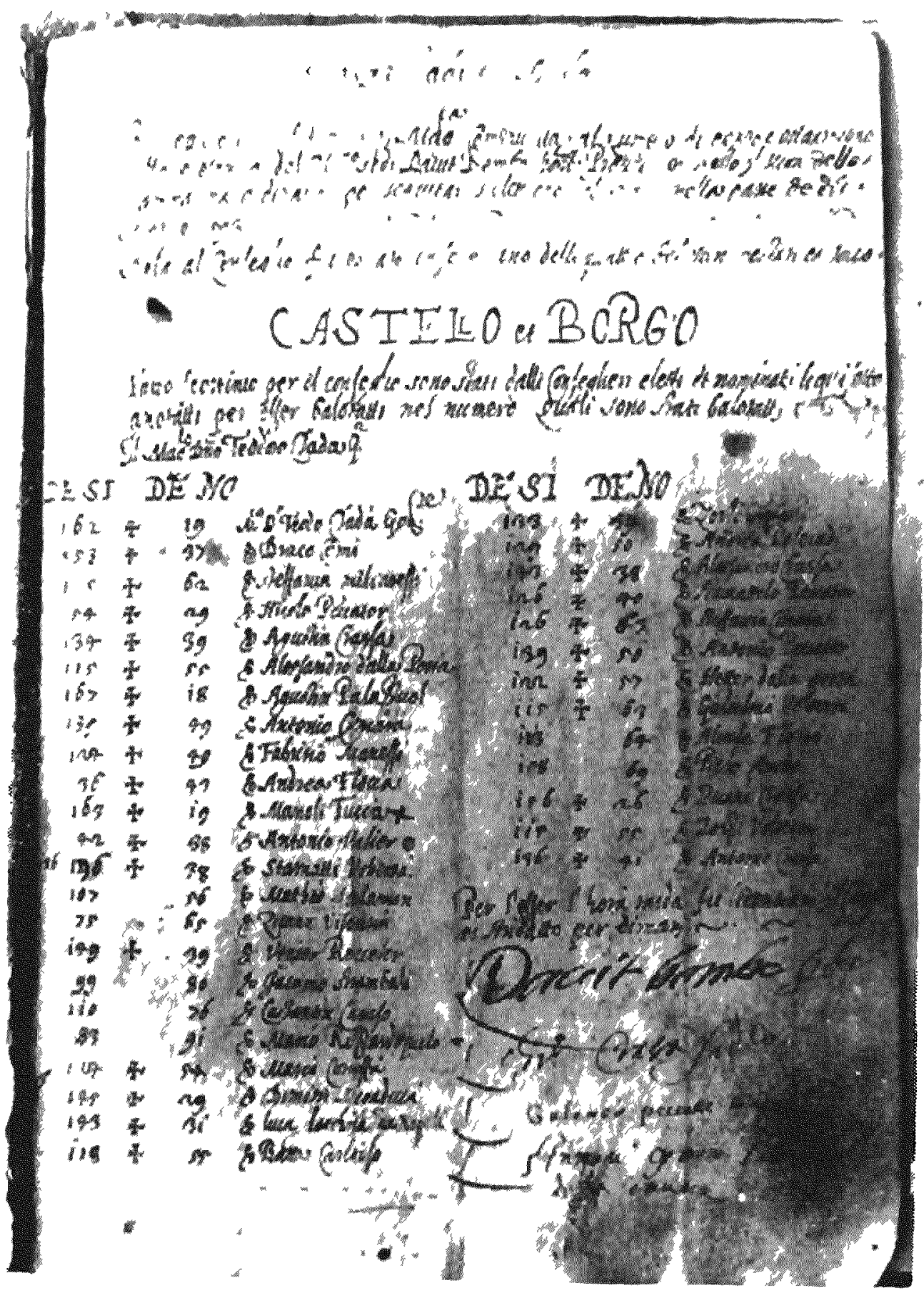

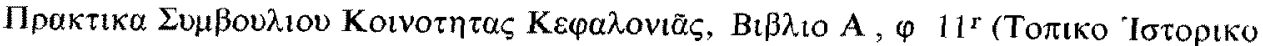

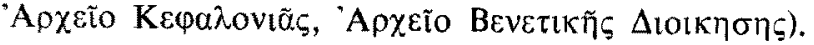


Пเv. 28

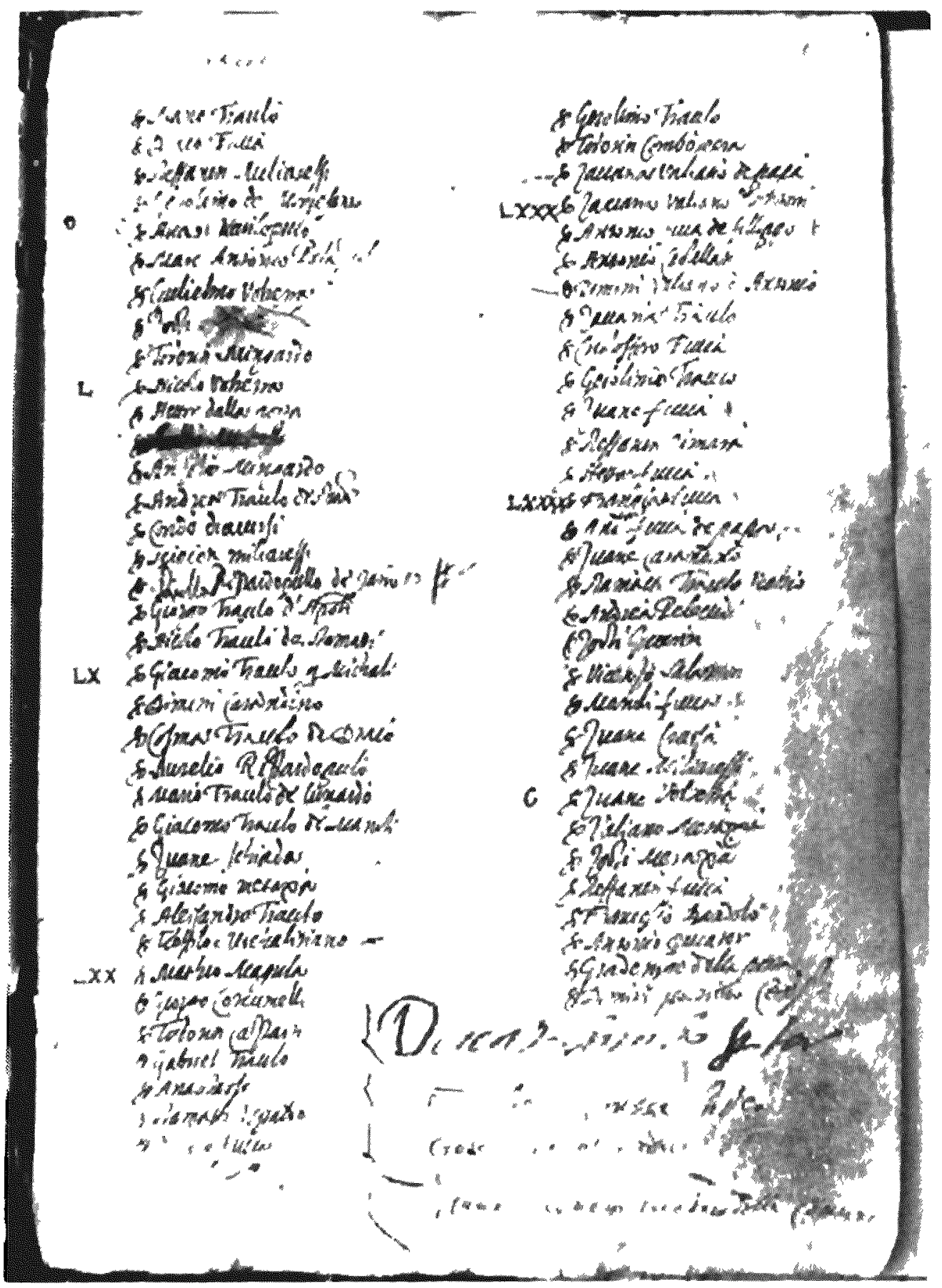

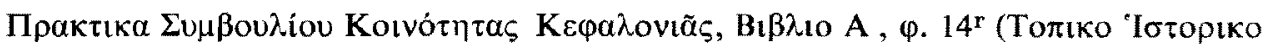
A 


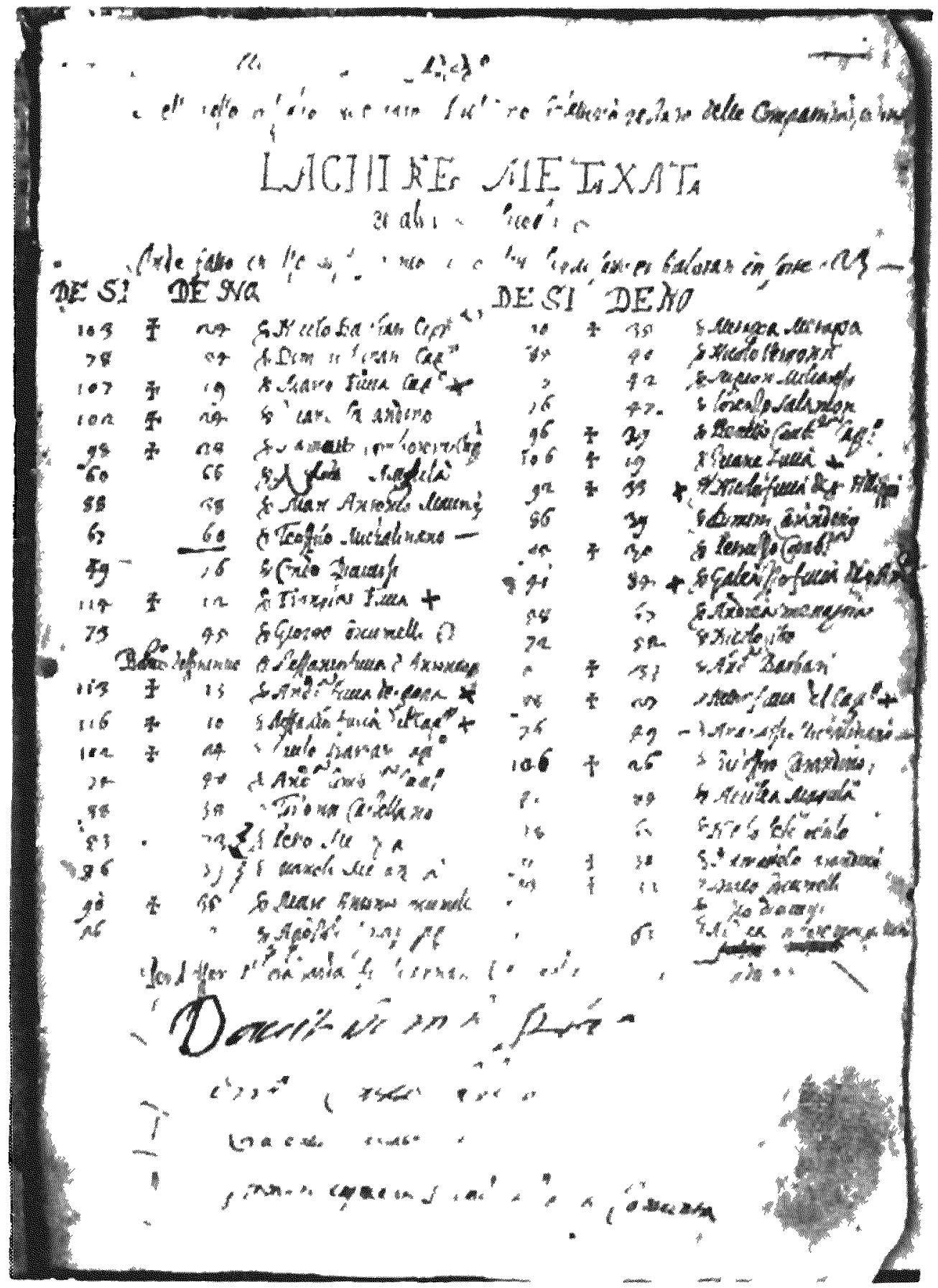

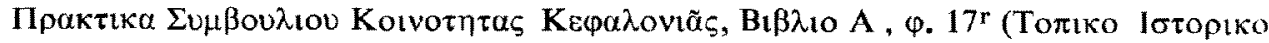

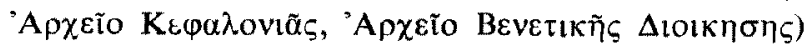


Miv. 30

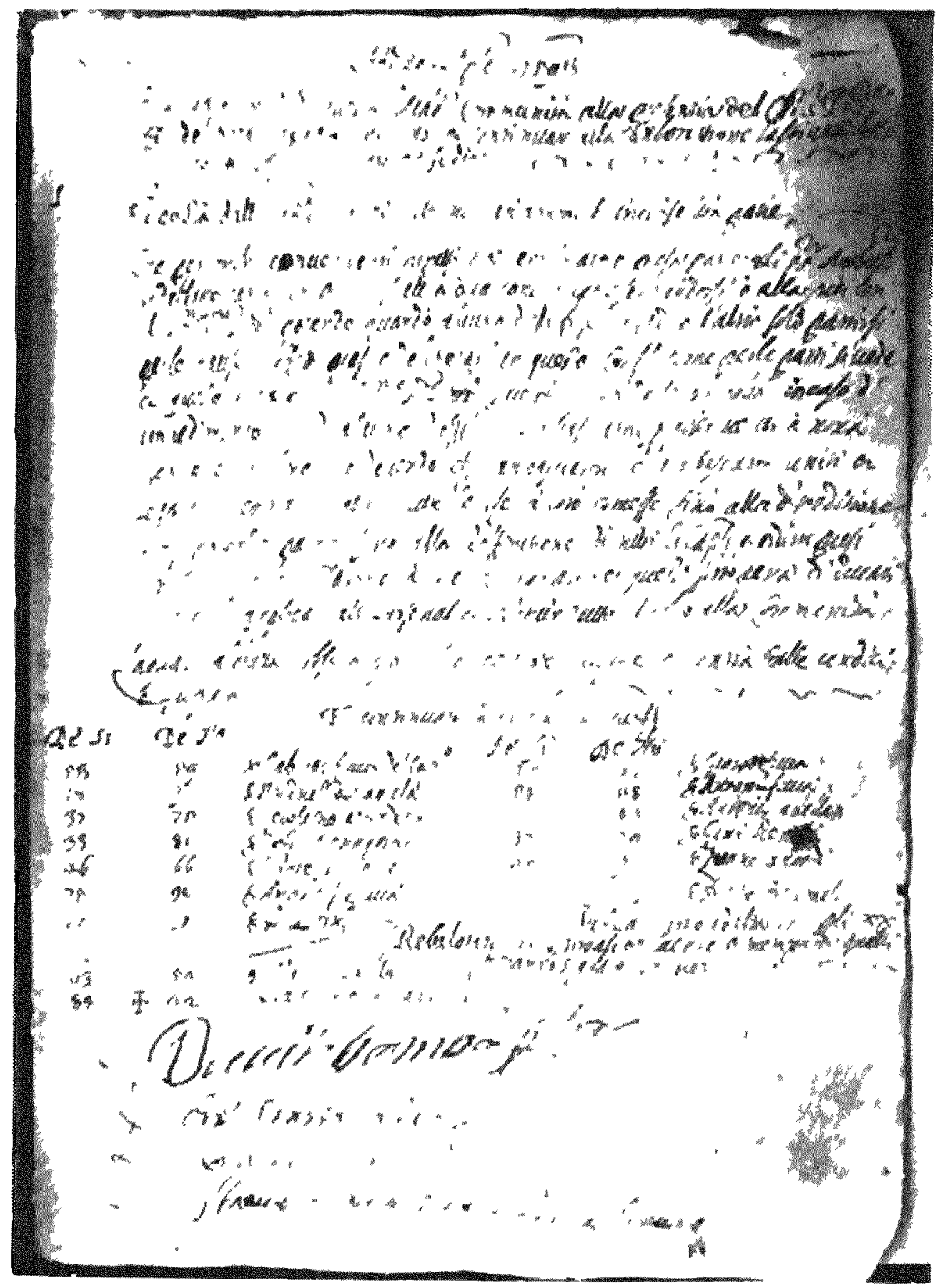

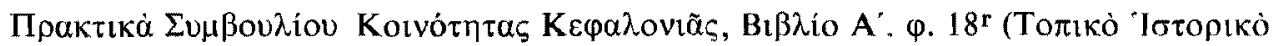

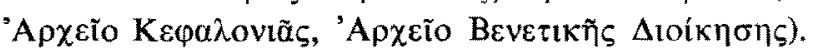

